\title{
New $\tau$-based evaluation of the hadronic contribution to the vacuum polarization piece of the muon anomalous magnetic moment
}

\author{
J. A. Miranda* and P. Roig $\oplus^{\dagger}$ \\ Departamento de Física, Centro de Investigación y de Estudios Avanzados del IPN, \\ Apartado Postal 14-740, 07000 Ciudad de México, México
}

(Received 20 September 2020; accepted 27 October 2020; published 8 December 2020)

\begin{abstract}
We revisit the isospin-breaking and electromagnetic corrections to the decay $\tau^{-} \rightarrow \pi^{-} \pi^{0} \nu_{\tau}$, which allow its use as input in the two-pion contribution to the (leading-order) hadronic vacuum polarization part of the muon anomalous magnetic moment. We extend a previous resonance chiral Lagrangian analysis, which included those operators saturating the next-to-leading-order chiral low-energy constants, by including the contributions appearing at the next order. As a result, we improve agreement between the two-pion tau decay and $e^{+} e^{-}$data and reduce the discrepancy between experiment and the Standard Model prediction of $a_{\mu}$ (using $\tau$ input) to the approximately $2 \sigma$ level.
\end{abstract}

DOI: $10.1103 /$ PhysRevD.102.114017

\section{INTRODUCTION}

The anomalous magnetic moment of the (first electron and then) muon $\left[a_{\mu} \equiv\left(g_{\mu}-2\right) / 2\right]$ has been crucial for the development of quantum field theory and the understanding of radiative corrections within it. Over the years, it has validated those computed in QED at increasing precision and (in the muon case) started probing the other Standard Model (SM) sectors, electroweak and QCD, setting also - and more interestingly-stringent constraints on new physics contributions. In the absence of any direct hint for heavy new particles or interactions at the LHC, clean observables both from experiment and theory—among which $a_{\mu}$ stands outare reinforced as a promising gate for the eagerly awaited further (indirect) discoveries in high-energy physics.

With the forthcoming measurement of $a_{\mu}$ at Fermi national accelerator laboratory (FNAL) [1], we will finally have an experimental update on the long-standing discrepancy (at 3 to 4 sigmas) between the SM prediction of this observable (recently refined in Ref. [2]) ${ }^{1}$ and its most accurate measurement, at Brookhaven national laboratory (BNL) [45]. On the theory side, a tremendous effort driven by the Muon $\mathrm{g}-2$ Theory Initiative ${ }^{2}$ has been reducing (and making more robust) the SM errors during the last few years,

jimiranda@ fis.cinvestav.mx

proig@fis.cinvestav.mx

${ }^{1}$ The SM prediction [2] is based on Refs. [3-37] (see also the last developments in Refs. [38-44]).

${ }^{2}$ Its website is https://muon-gm2-theory.illinois.edu/.

Published by the American Physical Society under the terms of the Creative Commons Attribution 4.0 International license. Further distribution of this work must maintain attribution to the author(s) and the published article's title, journal citation, and DOI. Funded by SCOAP. in order to profit maximally from the new data. In the near future, both the FNAL [1] and the Japan proton accelerator research complex [46] experiments will shrink the current experimental uncertainty $\left(63 \times 10^{-11}\right)$ by a factor 4 . A commensurate improvement on the theory error is essential in maximizing the reach on new physics of these measurements.

The SM uncertainty on $a_{\mu}\left(43 \times 10^{-11}\right)$ is saturated by that of the hadronic contributions, where the error of the dominant hadronic vacuum polarization $(H V P, L O)$ part has been reduced to $40 \times 10^{-11}$, versus $17 \times 10^{-11}$ of the lightby-light piece [2]. In turn, the HVP, LO contribution is dominated by the $\pi \pi$ cut (yielding approximately $73 \%$ of the overall value), where good-quality data of the corresponding $e^{+} e^{-}$hadronic cross sections [47-56] enable its computation by dispersive methods [57,58]. Alternatively, one can also use isospin-rotated $\tau \rightarrow \pi \pi \nu_{\tau}$ measurements with that purpose, as was put forward in large electronpositron collider times [59], despite that the required isospin-breaking (IB) corrections cannot be computed in a model-independent way presently. Still, while a lattice QCD computation of these is achieved, the authors find convenient testing the consistency of both extractions of $a_{\mu}^{\mathrm{HVP}, \mathrm{LO}_{\pi \pi}}$, in light of the tensions between different sets of $e^{+} e^{-} \rightarrow \pi^{+} \pi^{-}$data that have not been resolved so far [2].

In addition to the previous data-based determinations of $a_{\mu}^{\mathrm{HVP}, \mathrm{LO}}$, lattice QCD is also achieving computations with reduced errors, although not yet competitive with the $e^{+} e^{-}$evaluations [2]. One notable exception to this is the recent very accurate result $\left(53 \times 10^{-11}\right.$ error $)$ of the BMW Collaboration [60], according to which the difference with respect to the SM prediction is at the 1 sigma level.

Concerning the tau-based determination, Refs. [61,62] computed the required isospin-violating and electromagnetic 
corrections using resonance chiral theory $(R \chi T)[63,64]$, and Refs. [65,66] computed them using vector meson dominance (VMD). These series of articles were employed by Ref. [67] (updated in Refs. [68,69]), which, remarkably, found that the discrepancy of the SM prediction with the measurement is reduced substantially when tau data are employed. ${ }^{3}$ Notwithstanding, as precise measurements of $\sigma\left(e^{+} e^{-} \rightarrow\right.$ hadrons $)$ became available in the last 15 years, the $e^{+} e^{-}$-based evaluation gained preference over using tau data. Indeed, Ref. [2] concludes that "at the required precision to match the $e^{+} e^{-}$data, the present understanding of the IB (isospin-breaking) corrections to $\tau$ data is unfortunately not yet at a level allowing their use for the HVP dispersion integrals," despite Ref. [77] claiming that (the model-dependent) $\rho-\gamma$ mixing in the neutral channel makes it agree with the results in the charged current. It is the purpose of this work ${ }^{4}$ to extend previous $R \chi T$ analyses $[61,62]$ of the required IB corrections to dipion tau decays so that they can again be useful, when combined with $\sigma\left(e^{+} e^{-} \rightarrow \pi^{+} \pi^{-}(\gamma)\right)$, to increase the accuracy of the SM prediction of $a_{\mu}^{\mathrm{HVP}, \mathrm{LO}}$. In this spirit, we note that Jegerlehener [79] indeed combines both sets of data (using the IB corrections of Ref. [77]), which reduces the error of $a_{\mu}^{\mathrm{HVP}, \mathrm{LO}}$ by approximately $17 \%$ [79].

Within the global effort of the Muon g-2 theory initiative, we revisit in this paper the $R \chi T$ computations including operators that-in the chiral limit-start to contribute at $\mathcal{O}\left(p^{6}\right)$. This is possible by the knowledge acquired after the analyses of Cirigliano et al. [61,62] [where operators contributing at $\mathcal{O}\left(p^{4}\right)$ were considered], through a series of works studying operator product expansion (OPE) restrictions on $R \chi T$ couplings on several relevant threepoint Green's functions (and related form factors) [31,80101]. ${ }^{5,6}$ This procedure will also allow us to evaluate an uncertainty for the results by Cirigliano et al. [62], which is one of the main outcomes of this work, together with the new results, including operators that start contributing to the $\mathcal{O}\left(p^{6}\right)$ chiral low-energy constants (LECs).

The paper is organized as follows. In Sec. II, we review the main features of the $\tau^{-} \rightarrow \pi^{-} \pi^{0} \nu_{\tau} \gamma$ decays and split the model-independent part from the hadron form factors,

\footnotetext{
${ }^{3}$ The difference between the SM prediction of $a_{\mu}$ and the BNL measurement is $3.7 \sigma$ [2]. If isospin-rotated tau data are employed for $a_{\mu}^{\mathrm{HVP}, \mathrm{LO}}$, it amounts to $2.4 \sigma$ [69], instead. This difference could, in principle, be due to new physics effects, hinting at a lepton universality violation in the corresponding nonstandard vector and/or tensor couplings at low energies [70-72]. See the most updated discussions of its connection with $\alpha_{\mathrm{QED}}$ in the electroweak fit in Refs. [73-76].

${ }^{4}$ Currently, a lattice evaluation of IB for using tau data in $a_{\mu}^{\mathrm{HVP}, \mathrm{LO}}{ }_{\pi \pi}$ is in progress [78].

${ }^{5}$ See also, e.g., Refs. [102-108].

${ }^{6}$ Similar radiative corrections were computed for the $\tau \rightarrow \eta \pi \nu_{\tau} \gamma$ decays in $R \chi T$ [109], even though part of our contributions here were suppressed (and thus neglected) there because of G-parity.
}

computed in $R \chi T$ including new terms, subleading in the chiral expansion. We then recall the short-distance (SD) QCD constraints on the Lagrangian couplings and their phenomenological determinations and explain our estimation of the remaining free couplings, based on chiral counting. After that, in Sec. III, we recap the radiative corrections needed for the tau-based calculation of $a_{\mu}^{\mathrm{HVP}, \mathrm{LO}}$ and predict several observables for the processes where the real photon is detected together with the pion pair. Then, in Sec. IV, we evaluate $a_{\mu}^{\mathrm{HVP},\left.\mathrm{LO}\right|_{\pi \pi}}$ using tau data, which is the main result of this article. Finally, our conclusions are presented in Sec. V. Several Appendixes complement the main material, explaining how the coefficients dominating uncertainties were fitted, giving a full account of the kinematics, and providing with the complete expressions for the structure-dependent (axial-)vector form factors of the $\tau^{-} \rightarrow \pi^{-} \pi^{0} \nu_{\tau} \gamma$ decays.

\section{II. $\tau^{-} \rightarrow \pi^{-} \pi^{0} \gamma \nu_{\tau}$ DECAYS}

\section{A. Amplitude}

For the radiative decay $\tau^{-}(P) \rightarrow \pi^{-}\left(p_{-}\right) \pi^{0}\left(p_{0}\right) \nu_{\tau}(q) \gamma(k)$, we can split the contribution due to the bremsstrahlung off the initial tau lepton from the one coming from the hadronic part.

We write down the general structure for these processes $[62,110]$

$$
\begin{aligned}
T= & e G_{F} V_{u d}^{*} \epsilon^{\mu}(k)^{*}\left\{F_{\nu} \bar{u}(q) \gamma^{\nu}\left(1-\gamma_{5}\right)\left(m_{\tau}+\not P-\not k\right) \gamma_{\mu} u(P)\right. \\
& \left.+\left(V_{\mu \nu}-A_{\mu \nu}\right) \bar{u}(q) \gamma^{\nu}\left(1-\gamma_{5}\right) u(P)\right\},
\end{aligned}
$$

where $F_{\nu}=\left(p_{0}-p_{-}\right)_{\nu} f_{+}(s) / 2 P \cdot k$, with the charged pion vector form factor $f_{+}(s)$ defined through $\left\langle\pi^{0} \pi^{-}\left|\bar{d} \gamma^{\mu} u\right| 0\right\rangle=$ $\sqrt{2} f_{+}(s)\left(p_{-}-p_{0}\right)^{\mu}$ and $s=\left(p_{-}+p_{0}\right)^{2}$. Gauge invariance $\left(\epsilon_{\mu} \rightarrow \epsilon_{\mu}+k_{\mu}\right)$ implies the Ward identities

$$
k_{\mu} V^{\mu \nu}=\left(p_{-}-p_{0}\right)^{\nu} f_{+}(s), \quad k_{\mu} A^{\mu \nu}=0 .
$$

Imposing Eq. (2) and Lorentz invariance, we have the expression for the vector structure-dependent tensor

$$
\begin{aligned}
V^{\mu \nu}= & f_{+}\left[(P-q)^{2}\right] \frac{p_{-}^{\mu}\left(p_{-}+k-p_{0}\right)^{\nu}}{p_{-} \cdot k}-f_{+}\left[(P-q)^{2}\right] g^{\mu \nu} \\
& +\frac{f_{+}\left[(P-q)^{2}\right]-f_{+}(s)}{\left(p_{0}+p_{-}\right) \cdot k}\left(p_{0}+p_{-}\right)^{\mu}\left(p_{0}-p_{-}\right)^{\nu} \\
& +v_{1}\left(g^{\mu \nu} p_{-} \cdot k-p_{-}^{\mu} k^{\nu}\right)+v_{2}\left(g^{\mu \nu} p_{0} \cdot k-p_{0}^{\mu} k^{\nu}\right) \\
& +v_{3}\left(p_{0} \cdot k p_{-}^{\mu}-p_{-} \cdot k p_{0}^{\mu}\right) p_{-}^{\nu} \\
& +v_{4}\left(p_{0} \cdot k p_{-}^{\mu}-p_{-} \cdot k p_{0}^{\mu}\right)\left(p_{0}+p_{-}+k\right)^{\nu}
\end{aligned}
$$

and for the axial one

$$
\begin{aligned}
A^{\mu \nu}= & i a_{1} \epsilon^{\mu \nu \rho \sigma}\left(p_{0}-p_{-}\right)_{\rho} k_{\sigma}+i a_{2} W^{\nu} \epsilon^{\mu \lambda \rho \sigma} k_{\lambda} p_{-\rho} p_{0 \sigma} \\
& +i a_{3} \epsilon^{\mu \nu \rho \sigma} k_{\rho} W_{\sigma}+i a_{4}\left(p_{0}+k\right)^{\nu} \epsilon^{\mu \lambda \rho \sigma} k_{\lambda} p_{-\rho} p_{0 \sigma},
\end{aligned}
$$


where $W \equiv P-q=p_{-}+p_{0}+k$. We could use the basis given in Ref. [109], but instead we prefer a modified one that resembles the decomposition in Ref. [62] (see also Ref. [110]). These tensor structures depend on four vector $\left(v_{i}\right)$ and four axial-vector $\left(a_{i}\right)$ form factors. For the axial structure, the Schouten identity has been used.

Taking into account that $(P-q)^{2}=s+2\left(p_{0}+p_{-}\right) \cdot k$, the Low theorem [111] is manifestly satisfied:

$$
\begin{aligned}
V^{\mu \nu}= & f_{+}(s) \frac{p_{-}^{\mu}}{p_{-} \cdot k}\left(p_{-}-p_{0}\right)^{\nu}+f_{+}(s)\left(\frac{p_{-}^{\mu} k^{\nu}}{p_{-} \cdot k}-g^{\mu \nu}\right) \\
& +2 \frac{d f_{+}(s)}{d s}\left(\frac{p_{0} \cdot k}{p_{-} \cdot k} p_{-}^{\mu}-p_{0}^{\mu}\right)\left(p_{-}-p_{0}\right)^{\nu}+\mathcal{O}(k) .
\end{aligned}
$$

\section{B. Theoretical framework}

We will present in the following the model-dependent contributions to the $V_{\mu \nu}$ and $A_{\mu \nu}$ tensors. We will closely follow Ref. [62], extending it to include subleading terms in the chiral expansion. In this reference, a large- $N_{C}$ [112-114] inspired computation was carried out. Specifically, it was restricted to the dominant (for $N_{C} \rightarrow \infty$ ) tree-level diagrams, although the relevant loop corrections for the $\tau^{-} \rightarrow \pi^{-} \pi^{0} \nu_{\tau} \gamma$ decays-giving the $\rho$ (and $a_{1}$, for completeness) off-shell width ${ }^{7}$-were taken into account. ${ }^{8}$ Also, given the limited phase space of tau decays and the fact that the region $E \lesssim M_{\rho}+\Gamma_{\rho}$ is the most important one for the IB corrections needed for $a_{\mu}^{\mathrm{HVP}, \mathrm{LO}_{\pi \pi}}$ [62], the contribution of the $\rho(1450)$ and other heavier resonances was neglected in this reference (despite the fact that, in the large- $N_{C}$ limit, there is an infinite tower of resonances per channel), as we will also do. ${ }^{9}$ Within this setting, our computation will include all $R \chi T$ operators contributing to the $\mathcal{O}\left(p^{6}\right)$ chiral low-energy constants. Our results agree with those in Ref. [62], providing the new contributions with resonance operators that are suppressed by one chiral order in the low-energy limit (where possible, our computations have been checked against the results in Ref. [109]).

As explained in Ref. [62], this procedure warrants the correct low-energy limit (as given by chiral perturbation theory [126-130]) and includes consistently the most general pion and photon interactions with the lightest resonances. Demanding the known QCD SD constraints results in relations among the Lagrangian couplings, and chiral counting can be employed to estimate those still

\footnotetext{
${ }^{7}$ We will introduce them following Ref. [115] for the $\rho(770)$ and Refs. [86,116] for the $a_{1}(1260)$ resonances.

${ }^{8}$ See Refs. [117-123] for next-to-leading-order computations in $1 / N_{C}$, allowing to include the scale dependence of the chiral pertubation theory LECs in the low-energy limit of $R \chi T$.

${ }^{9}$ Nevertheless, we will include the dominant effect of the $\rho(1450)$ and $\rho(1700)$ resonances in our dispersive pion form factor $[124,125]$ and check the negligible impact of heavier resonances in the $v_{i}$ and $a_{i}$ form factors in our analysis.
}

unconstrained after using phenomenological information. It should then provide an accurate description of the $\tau^{-} \rightarrow \pi^{-} \pi^{0} \nu_{\tau} \gamma$ decays for $s \lesssim 1 \mathrm{GeV}^{2}$, which gives approximately $99.8 \%$ of the whole $a_{\mu}^{\mathrm{HVP},\left.\mathrm{LO}\right|_{\pi \pi}}$ contribution.

\section{Vector form factors}

Within $R \chi T$ [63,64,83,88], the diagrams contributing to the vector form factors of the $\tau^{-} \rightarrow \pi^{-} \pi^{0} \gamma \nu_{\tau}$ decays including operators that start contributing to the $\mathcal{O}\left(p^{6}\right)$ LECs are shown in Figs. $1-3 .^{10}$ The first three diagrams in Fig. 1 and the first diagram in Fig. 2 contribute to the pion vector form factor entering the structure-independent (SI) piece $^{11}$

$$
\begin{aligned}
f_{+}(s)= & 1+\frac{G_{V} F_{V}}{F^{2}} \frac{s}{m_{\rho}^{2}-s} \\
& +\frac{\sqrt{2} F_{V} s}{F^{2}\left(m_{\rho}^{2}-s\right)}\left[2\left(2 \lambda_{8}^{V}+\lambda_{9}^{V}+2 \lambda_{10}^{V}\right) m_{\pi}^{2}-s \lambda_{21}^{V}\right] \\
& +\frac{2 \sqrt{2} G_{V} s}{F^{2}\left(m_{\rho}^{2}-s\right)}\left[4 \lambda_{6}^{V} m_{\pi}^{2}-s \lambda_{22}^{V}\right] \\
& +\frac{4 s}{F^{2}\left(m_{\rho}^{2}-s\right)}\left[4 \lambda_{6}^{V} m_{\pi}^{2}-s \lambda_{22}^{V}\right] \\
& \times\left[2\left(2 \lambda_{8}^{V}+\lambda_{9}^{V}+2 \lambda_{10}^{V}\right) m_{\pi}^{2}-s \lambda_{21}^{V}\right] .
\end{aligned}
$$

The contribution of both the last diagram in Fig. 1 and the last diagram in Fig. 2 vanishes for a real photon, as the corresponding $\left[f_{+}(0)=1\right.$ part $]$ contribution is already in the SI piece. We note we are using $F \sim 92 \mathrm{MeV}$ for the pion decay constant and that QCD OPE constraints $\lambda_{21}^{V}=\lambda_{22}^{V}=0$ [83]. In fact, we will see in Sec. II E that all modifications induced by the $\lambda_{i}^{V}$ couplings to $f_{+}(s)(6)$ vanish once SD QCD constraints are accounted for.

For the vector form factors, we get

$$
\begin{aligned}
& v_{1}=v_{1}^{0}+v_{1}^{R}+v_{1}^{R R}+v_{1}^{R R R}+v_{G I 1}^{R+R R}, \\
& v_{2}=v_{2}^{0}+v_{2}^{R}+v_{2}^{R R}+v_{2}^{R R R}+v_{G I 2}^{R+R R}, \\
& v_{3}=v_{3}^{0}+v_{3}^{R}+v_{3}^{R R}+v_{3}^{R R R}+v_{G I 3}^{R+R R}, \\
& v_{4}=v_{4}^{0}+v_{4}^{R}+v_{4}^{R R}+v_{4}^{R R R}+v_{G I 4}^{R+R R},
\end{aligned}
$$

where $v_{i}^{0}$ is the contribution in Ref. [62] ( $D_{R}^{-1}$ stands for the inverse resonance propagator), ${ }^{12}$

\footnotetext{
${ }^{10}$ The contributions involving scalar and pseudoscalar resonances are discussed at the end of Sec. II C.

${ }^{11}$ Relevant $R \chi T$ couplings are introduced after Eq. (7) and in Sec. II E below.

${ }^{12}$ We recall that $F_{R}$ gives the coupling of the $R=V, A$ resonance to the $r=v, a$ external current and the $\rho \pi \pi$ vertex receives contributions both from $F_{V}$ and $G_{V}$.
} 

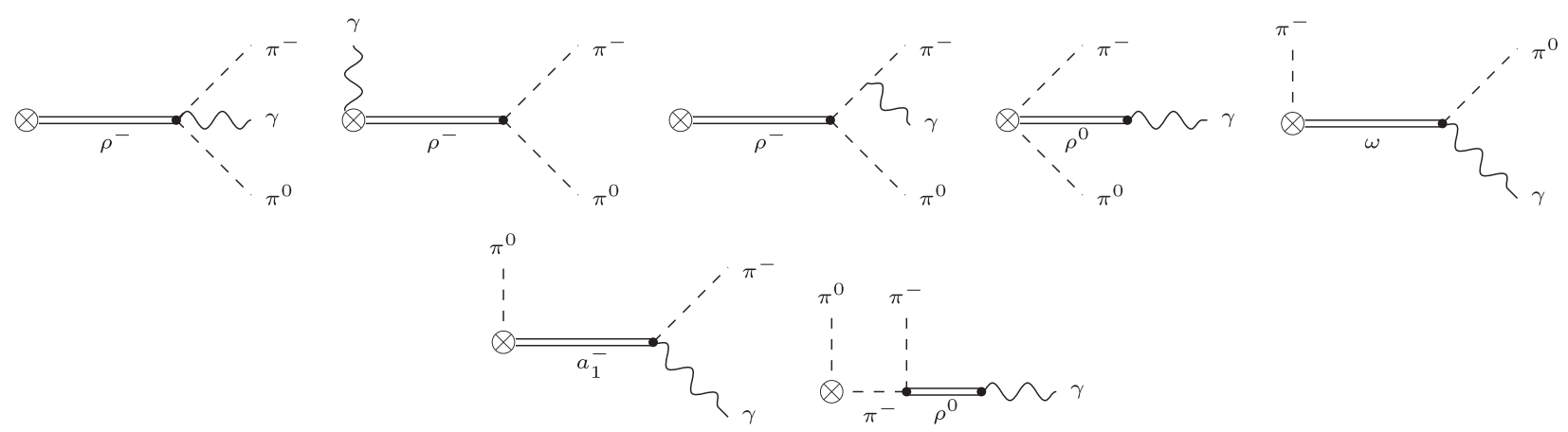

FIG. 1. One-resonance exchange contributions from the $R \chi T$ to the vector form factors of the $\tau^{-} \rightarrow \pi^{-} \pi^{0} \gamma \nu_{\tau}$ decays.
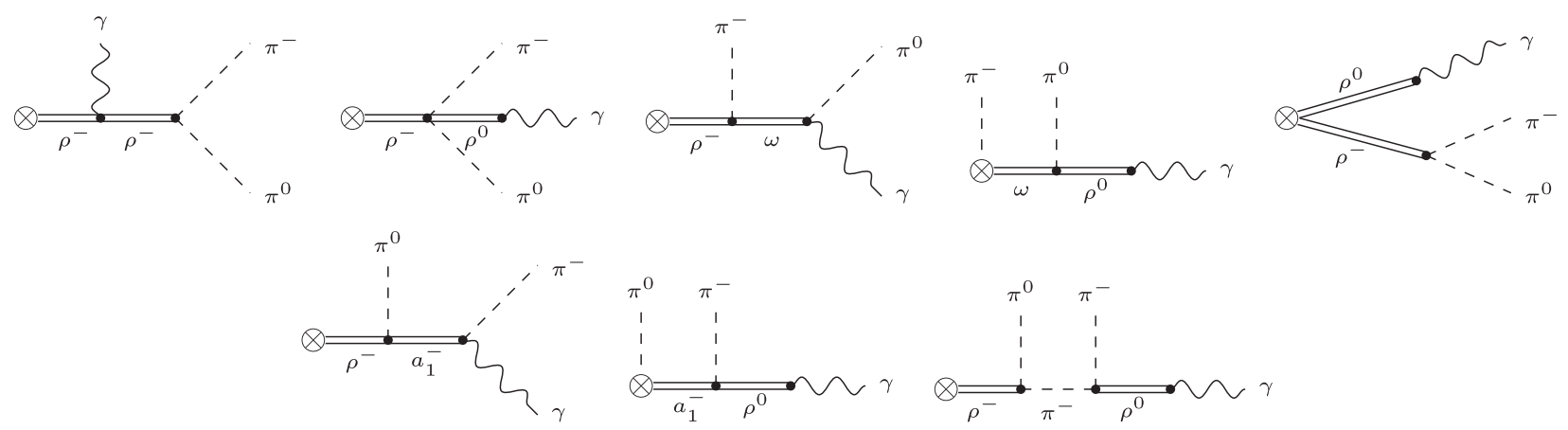

FIG. 2. Two-resonance exchange contributions from the $R \chi T$ to the vector form factors of the $\tau^{-} \rightarrow \pi^{-} \pi^{0} \gamma \nu_{\tau}$ decays.

$$
\begin{aligned}
v_{1}^{0} \equiv & \frac{F_{V} G_{V}}{F^{2} M_{\rho}^{2}}\left(2+2 M_{\rho}^{2} D_{\rho}^{-1}\left[(P-q)^{2}\right]+s D_{\rho}^{-1}(s)+s M_{\rho}^{2} D_{\rho}^{-1}(s) D_{\rho}^{-1}\left[(P-q)^{2}\right]\right) \\
& +\frac{F_{V}^{2}}{2 F^{2} M_{\rho}^{2}}\left(-1-M_{\rho}^{2} D_{\rho}^{-1}\left[(P-q)^{2}\right]+(P-q)^{2} D_{\rho}^{-1}\left[(P-q)^{2}\right]\right) \\
& +\frac{F_{A}^{2}}{F^{2} M_{a_{1}}^{2}}\left(M_{a_{1}}^{2}-m_{\pi}^{2}+\frac{1}{2} s\right) D_{a_{1}}^{-1}\left[\left(p_{-}+k\right)^{2}\right], \\
v_{2}^{0} \equiv & \frac{F_{V} G_{V} s}{F^{2} M_{\rho}^{2}}\left(-D_{\rho}^{-1}(s)-M_{\rho}^{2} D_{\rho}^{-1}(s) D_{\rho}^{-1}\left[(P-q)^{2}\right]\right) \\
& +\frac{F_{V}^{2}}{2 F^{2} M_{\rho}^{2}}\left(-1-M_{\rho}^{2} D_{\rho}^{-1}\left[(P-q)^{2}\right]-(P-q)^{2} D_{\rho}^{-1}\left[(P-q)^{2}\right]\right) \\
& +\frac{F_{A}^{2}}{F^{2} M_{a_{1}}^{2}}\left(M_{a_{1}}^{2}-m_{\pi}^{2}-k \cdot p D_{-}^{-1}\left[\left(p_{-}+k\right)^{2}\right],\right. \\
v_{3}^{0} \equiv & \frac{F_{A}^{2}}{F^{2} M_{a_{1}}^{2}} D_{a_{1}}^{-1}\left[\left(p_{-}+k\right)^{2}\right], \\
v_{4}^{0} \equiv & -\frac{2 F_{V} G_{V}}{F^{2}} D_{\rho}^{-1}(s) D_{\rho}^{-1}\left[(P-q)^{2}\right]+\frac{F_{V}^{2}}{F^{2} M_{\rho}^{2}} D_{\rho}^{-1}\left[(P-q)^{2}\right],
\end{aligned}
$$

and $v_{i}^{R}, v_{i}^{R R}, v_{i}^{R R R}$, and $v_{G I i}^{R+R R 13}$ correspond to contributions including operators which do not contribute to the next-toleading-order (NLO) chiral LECs. Because of their length, the expressions for these form factors are in Appendix C. In writing the new contributions to $v_{i}$, the basis given in Ref. [83] has been used for the even-intrinsic parity operators (with couplings $\lambda_{i}^{X}$ ), and the basis given in Ref. [88] has been employed for the odd-intrinsic parity operators ( $\kappa_{i}^{X}$ couplings). Both sets of $\lambda_{i}^{X}$ and $\kappa_{i}^{X}$ couplings have dimensions of inverse energy.

\footnotetext{
${ }^{13}$ In general, diagrams are gauge invariant by themselves. Those giving the contribution $v_{G I i}^{R+R R}$ need to be summed to achieve gauge invariance. These are the first three diagrams in Fig. 1 and the first diagram in Fig. 2.
} 


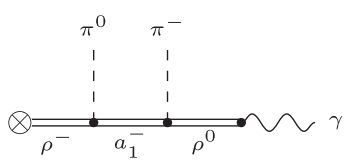

FIG. 3. Three-resonance exchange contributions from the $R \chi T$ to the vector form factors of the $\tau^{-} \rightarrow \pi^{-} \pi^{0} \gamma \nu_{\tau}$ decays.

Including operators with at most one resonance, only the contribution from the exchange of $\rho$ and $a_{1}$ resonances on the vector form factor appeared [62]. Allowing for multiresonance operators, we also have contributions with $\omega$ exchange, coming from the odd-intrinsic parity sector, for both vector and axial-vector form factors (as well as resonance contributions on the axial form factor, absent in Ref. [62]). Apparently, such $\omega$ contributions were responsible for the larger effect of the IB corrections obtained in Refs. [65,66] with respect to Refs. [61,62]. As a result, Ref. [67] (and later evaluations by this group) ascribed an error to these corrections covering both contradictory evaluations. As we include (among others) contributions with an $\omega-\rho-\pi$ vertex in this work, closer agreement with the VMD evaluation should, in principle, be expected.

We have verified that all diagrams including scalar mesons vanish in the isospin symmetry limit. We point out that all contributions involving pseudoscalar mesons can be obtained from those with an axial-vector resonance by replacing it by a pseudoscalar resonance. Then, at leading chiral order, the saturation of the LECs by spin-1 mesons [63] shows that diagrams including pseudoscalar resonances are suppressed. If we assume that this feature also holds at the next chiral order, then pseudoscalar resonance exchanges could be safely neglected. ${ }^{14}$

\section{Axial-vector form factors}

The axial form factors at chiral $\mathcal{O}\left(p^{4}\right)$ get contributions from the Wess-Zumino-Witten functional [131,132]:

$$
a_{1}^{0} \equiv \frac{1}{8 \pi^{2} F^{2}}, \quad a_{2}^{0} \equiv \frac{-1}{4 \pi^{2} F^{2}\left[(P-q)^{2}-m_{\pi}^{2}\right]} .
$$

The diagrams that receive contributions due to the anomaly are shown in Fig. $4 .{ }^{15}$

For the axial form factors (see Figs. 5-7), we get

$$
\begin{aligned}
& a_{1}=a_{1}^{0}+a_{1}^{R}+a_{1}^{R R}+a_{1}^{R R R}, \\
& a_{2}=a_{2}^{0}+a_{2}^{R}+a_{2}^{R R}+a_{2}^{R R},
\end{aligned}
$$

\footnotetext{
${ }^{14}$ Since contributions from scalar and pseudoscalar resonances are suppressed, we will neglect them for the axial form factors in the next section.

${ }^{15}$ The first diagram, when coupled to a vector current, contributes to the SI piece in $V^{\mu \nu}$.
}

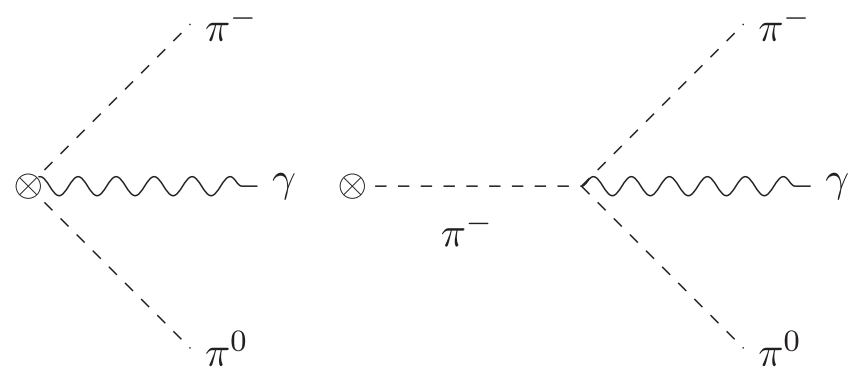

FIG. 4. Anomalous diagrams contributing to the axial tensor amplitude $A^{\mu \nu}$ at $\mathcal{O}\left(p^{4}\right)$.

$$
\begin{aligned}
& a_{3}=a_{3}^{R}+a_{3}^{R R}+a_{3}^{R R R}, \\
& a_{4}=a_{4}^{R}+a_{4}^{R R}+a_{4}^{R R R},
\end{aligned}
$$

where $a_{i}^{R}, a_{i}^{R R}$, and $a_{i}^{R R R}$ include $\mathcal{O}\left(p^{6}\right)$ vertices. Because of their length, the expressions for these form factors appear in Appendix D.

\section{E. SD constraints}

Including operators which start contributing to the $\mathcal{O}\left(p^{6}\right)$ LECs, we have now so many parameters (see Table I) allowed by the discrete symmetries of QCD and chiral symmetry that, in practice, prevent making phenomenological predictions. It is possible to find relations between these couplings by means of SD properties of QCD and its OPE. We summarize these results in this section.

For the parameters contributing to two-point Green's functions (and related form factors), the constraints $[63,64,133-137]$

$$
\begin{array}{rlrl}
F_{V} G_{V} & =F^{2}, & F_{V}^{2}-F_{A}^{2}=F^{2}, \\
F_{V}^{2} M_{V}^{2} & =F_{A}^{2} M_{A}^{2}, \quad 4 c_{d} c_{m}=F^{2}, \\
8\left(c_{m}^{2}-d_{m}^{2}\right) & =F^{2}, \quad c_{m}=c_{d}=\sqrt{2} d_{m}=F / 2
\end{array}
$$

are set, respectively, by the known asymptotic behavior of the pion vector form factor, the $V-A$ correlator (yielding the Weinberg sum rules), the scalar form factor, and the $S-P$ correlator.

We note that the vanishing of the axial pion form factor (giving the $\pi$-to- $\gamma$ matrix element) at infinite momentum transfer demands-if only the original $R \chi T$ Lagrangian [64] is used $-2 F_{V} G_{V}=F_{V}^{2}$. This, together with the two first equations in Eq. (10), determine

$$
F_{V}=\sqrt{2} F, \quad G_{V}=\frac{F}{\sqrt{2}}, \quad F_{A}=F,
$$

all in terms of the pion decay constant. These relations were employed in Ref. [62]. We emphasize that-once operators contributing to the NLO chiral LECs are 

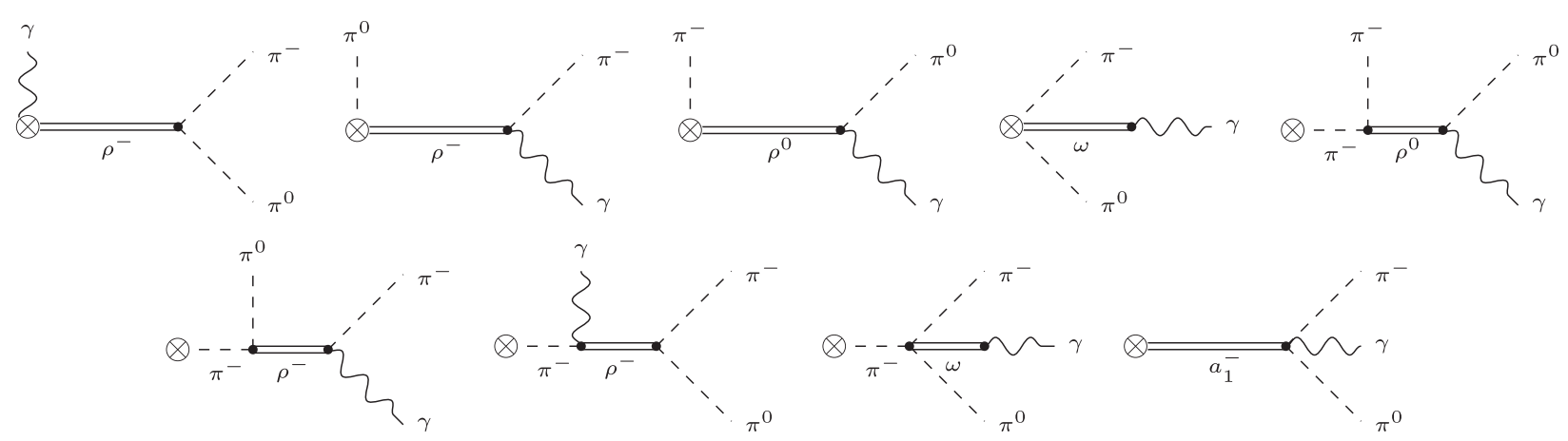

FIG. 5. One-resonance exchange contributions from the $R \chi T$ to the axial-vector form factors of the $\tau^{-} \rightarrow \pi^{-} \pi^{0} \gamma \nu_{\tau}$ decays.
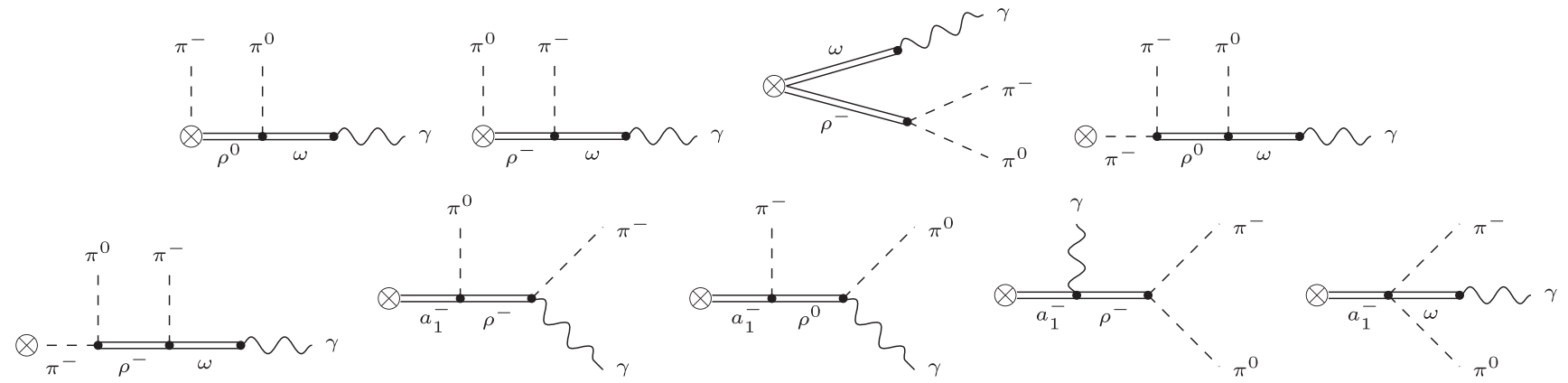

FIG. 6. Two-resonance exchange contributions from the $R \chi T$ to the axial-vector form factors of the $\tau^{-} \rightarrow \pi^{-} \pi^{0} \gamma \nu_{\tau}$ decays.
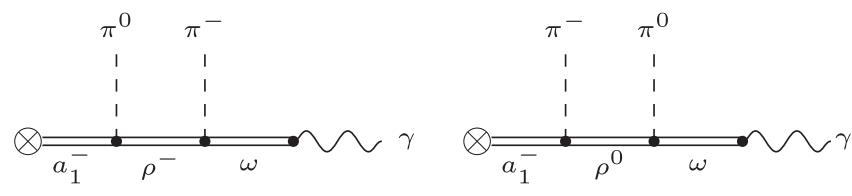

FIG. 7. Three-resonance exchange contributions from the $R \chi T$ to the axial-vector form factors of the $\tau^{-} \rightarrow \pi^{-} \pi^{0} \gamma \nu_{\tau}$ decays.

considered $[83,88]$ — the relations (11) no longer hold true (see Ref. [94]). Seen from another perspective, consistent sets of SD relations on $n$-point Green's functions vary with $n$. For $n=2$, one has the set (11) [63]. We shall also consider the set obtained for $n=3[83,88,94]$ (where operators with more than one resonance field start to appear) in the following. We will come back to discussing the actual values of the $F_{V}, G_{V}$, and $F_{A}$ couplings before closing this section, as they are essential to assess the error associated to the IB corrections computed in Ref. [62].

Now, we consider $R \chi T$ operators which do not contribute to the NLO chiral LECs. For the even-intrinsic parity sector $[83,98],{ }^{16}$

$$
\begin{gathered}
\lambda_{13}^{P}=0, \quad \lambda_{17}^{S}=\lambda_{18}^{S}=0, \\
\lambda_{17}^{A}=0, \quad \lambda_{6}^{V}=\lambda_{21}^{V}=\lambda_{22}^{V}=0,
\end{gathered}
$$

\footnotetext{
${ }^{16}$ The corresponding coefficients are denoted $\lambda_{i}^{R}$, with the upper index showing the resonance fields involved.
}

using these SD constraints in Eq. (6) and the BrodskyLepage behavior $[138,139]$ of $f_{+}(s)$, we get

$$
2 \lambda_{8}^{V}+\lambda_{9}^{V}+2 \lambda_{10}^{V}=0
$$

The study of the $\langle V A P\rangle$ and $\langle S P P\rangle$ Green's functions yields the following restrictions on the resonance couplings [81-83] [the Weinberg sum rules in Eq. (10) were used below]:

$$
\begin{aligned}
& \sqrt{2} \lambda_{0}=-4 \lambda_{1}^{V A}-\lambda_{2}^{V A}-\frac{\lambda_{4}^{V A}}{2}-\lambda_{5}^{V A}=\frac{1}{2 \sqrt{2}}\left(\lambda^{\prime}+\lambda^{\prime \prime}\right) \\
& \sqrt{2} \lambda^{\prime}=\lambda_{2}^{V A}-\lambda_{3}^{V A}+\frac{\lambda_{4}^{V A}}{2}+\lambda_{5}^{V A}=\frac{F_{V}}{2 \sqrt{F_{V}^{2}-F^{2}}}, \\
& \sqrt{2} \lambda^{\prime \prime}=\lambda_{2}^{V A}-\frac{\lambda_{4}^{V A}}{2}-\lambda_{5}^{V A}=\frac{2 F^{2}-F_{V}^{2}}{2 F_{V} \sqrt{F_{V}^{2}-F^{2}}}, \\
& \lambda_{1}^{P V}=-4 \lambda_{2}^{P V}=-\frac{F^{2}}{4 \sqrt{2} d_{m} F_{V}}, \quad \lambda_{1}^{P A}=\frac{F^{2}}{16 \sqrt{2} d_{m} \sqrt{F_{V}^{2}-F^{2}}} .
\end{aligned}
$$

For the odd-intrinsic parity sector $[88],{ }^{17}$

\footnotetext{
${ }^{17}$ The corresponding coefficients are denoted $\kappa_{i}^{R}$, with the upper index showing the resonance fields involved.
} 


$$
\begin{aligned}
\kappa_{14}^{V} & =\frac{N_{C}}{256 \sqrt{2} \pi^{2} F_{V}}, \quad 2 \kappa_{12}^{V}+\kappa_{16}^{V}=-\frac{N_{C}}{32 \sqrt{2} \pi^{2} F_{V}}, \\
\kappa_{17}^{V} & =-\frac{N_{C}}{64 \sqrt{2} \pi^{2} F_{V}}, \quad \kappa_{5}^{P}=0, \\
\kappa_{2}^{V V} & =\frac{F^{2}+16 \sqrt{2} d_{m} F_{V} \kappa_{3}^{P V}}{32 F_{V}^{2}}-\frac{N_{C} M_{V}^{2}}{512 \pi^{2} F_{V}^{2}}, \\
8 \kappa_{2}^{V V}-\kappa_{3}^{V V} & =\frac{F^{2}}{8 F_{V}^{2}} .
\end{aligned}
$$

The analysis of the $\langle V A S\rangle$ Green's function yields [88]

$$
\begin{array}{r}
\kappa_{2}^{S}=\kappa_{14}^{A}=0, \quad \kappa_{4}^{V}=2 \kappa_{15}^{V}, \quad \kappa_{6}^{V A}=\frac{F^{2}}{32 F_{A} F_{V}}, \\
F_{V}\left(2 \kappa_{1}^{S V}+\kappa_{2}^{S V}\right)=2 F_{A} \kappa_{1}^{S A}=\frac{F^{2}}{16 \sqrt{2} c_{m}},
\end{array}
$$

and through the study of the $\langle V V A\rangle$ Green's function in Ref. [31],

$$
F_{V} \kappa_{5}^{V A}=-\frac{N_{C} M_{V}^{2}}{64 \pi^{2} F_{A}} .
$$

A comparison between two basis for the odd-intrinsic operators [80,88] was given in Ref. [94], which is consistent with those in Eq. $(15)^{18}$ :

$$
\begin{aligned}
M_{V}\left(2 \kappa_{12}^{V}+4 \kappa_{14}^{V}+\kappa_{16}^{V}-\kappa_{17}^{V}\right) & =4 c_{3}+c_{1}=0, \\
M_{V}\left(2 \kappa_{12}^{V}+\kappa_{16}^{V}-2 \kappa_{17}^{V}\right) & =c_{1}-c_{2}+c_{5}=0, \\
-M_{V} \kappa_{17}^{V} & =c_{5}-c_{6}=\frac{N_{C} M_{V}}{64 \sqrt{2} \pi^{2} F_{V}}, \\
M_{V} \kappa_{15}^{V} & =c_{4}, \\
8 \kappa_{2}^{V V} & =d_{1}+8 d_{2}=\frac{F^{2}}{8 F_{V}^{2}}-\frac{N_{C} M_{V}^{2}}{64 \pi^{2} F_{V}^{2}}, \\
\kappa_{3}^{V V} & =d_{3}=-\frac{N_{C}}{64 \pi^{2}} \frac{M_{V}^{2}}{F_{V}^{2}}, \\
1+\frac{32 \sqrt{2} F_{V} d_{m} \kappa_{3}^{P V}}{F^{2}} & =0, \\
F_{V}^{2} & =3 F^{2} .
\end{aligned}
$$

For the even- and odd-intrinsic parity sectors, there are 115 even-intrinsic parity sector (EIP) +67 odd-intrinsic parity sector $(\mathrm{OIP})=182$ operators saturating the $\mathcal{O}\left(p^{6}\right)$ LECs, but only a few of them contribute to a given process. The form factors of the $\tau^{-} \rightarrow \pi^{-} \pi^{0} \gamma \nu_{\tau}$ decays at $\mathcal{O}\left(p^{6}\right)$ are given by $32(\mathrm{EIP})+23(\mathrm{OIP})=55$ operators (Table I). Taking into account the relations in Eqs. (12)-(18), we get $24(\mathrm{EIP})+17(\mathrm{OIPP})=41$ undetermined couplings. To estimate the unknown parameters, we basically followed

\footnotetext{
${ }^{18}$ We note, particularly, the last of these equations, which is at odds with Eq. (11).
}

TABLE I. Operators contributing at $\mathcal{O}\left(p^{6}\right)$ to the vector and axial-vector form factors.

Even-intrinsic parity [83]

$\hat{O}_{i}^{V} \quad 6,7,8,9,10,12,13,14,15,16,17,18,19,20,21,22$

$\hat{O}_{i}^{A} \quad 4,12,13,15,16,17$

$\hat{O}_{i}^{V V} \quad 2,3,4,5,7$

$\hat{O}_{i}^{V A} \quad 1,2,3,4,5$

Odd-intrinsic parity [88]
$\hat{O}_{i \mu \nu \alpha \beta}^{V}$
$\hat{O}_{i \mu \nu \alpha \beta}^{A}$
$1,2,3,6,7,8,9,10,11,12,14,16,17$
$\hat{O}_{i \mu \nu \alpha \beta}^{V V}$
$5,6,7$
$\hat{O}_{i \mu \nu \alpha \beta}^{V A}$
2, 3,4
$2,3,4,5$

(but for the results in Appendix A) the strategy devised in Ref. [109]. We will restore to the available phenomenological information on these couplings and estimatebased on chiral counting - those for which we lack it.

Equation (14) leaves two $\lambda_{i}^{V A}$ couplings undetermined; the numerical values of the restricted combinations (see their definitions in terms of the $\lambda_{i}^{V A}$ in Ref. [81]) are

$$
\lambda^{\prime} \sim 0.4, \quad \lambda^{\prime \prime} \sim-0.14, \quad \lambda_{0} \sim 0.07 .
$$

Since the same linear combination of $\lambda_{4}^{V A}$ and $\lambda_{5}^{V A}$ is in all couplings in Eq. (19), we choose $\lambda_{4}^{V A}$ as independent. By similar reasons, we take $\lambda_{2}^{V A}$ as the other independent coupling. Based on Eq. (19), we conservatively estimate $\left|\lambda_{2}^{V A}\right| \sim\left|\lambda_{4}^{V A}\right| \leq 0.4$.

According to Ref. [83] the $\lambda_{i}^{X}$ couplings can be estimated from low-energy couplings $C_{i}^{R}$ of the $\mathcal{O}\left(p^{6}\right) \chi P T$ Lagrangian as ${ }^{19}$

$$
\begin{aligned}
\left|\lambda_{i}^{V}\right| & \sim \frac{3 M_{V}^{2}}{2 F} C_{i}^{R} \sim 0.025 \mathrm{GeV}^{-1}, \\
\left|\lambda_{i}^{V V}\right| & \sim \frac{M_{V}^{4}}{2 F^{2}} C_{i}^{R} \sim 0.1,
\end{aligned}
$$

where we take the relation $\left|C_{i}^{R}\right| \sim \frac{1}{F^{2}(4 \pi)^{4}}$ linked to $\left|L_{i}^{R}\right| \sim \frac{1}{(4 \pi)^{2}} \sim 5 \times 10^{-3}$, which corresponds to the typical size of the $\mathcal{O}\left(p^{4}\right)$ LECs. This sets a reasonable upper bound on $\left|\lambda_{i}^{V}\right| \sim\left|\lambda_{i}^{A}\right| \lesssim 0.025 \mathrm{GeV}^{-1}$ and $\left|\lambda_{i}^{V V}\right| \sim\left|\lambda_{i}^{V A}\right| \lesssim 0.1$.

For the anomalous sector, we have the following predictions from Eq. (18): $-M_{V} \kappa_{17}^{V}=c_{5}-c_{6} \sim 0.016$, $8 \kappa_{2}^{V V}=d_{1}+8 d_{2} \sim-0.070 \quad$ and $\quad \kappa_{3}^{V V}=d_{3} \sim-0.112$. There is a sign ambiguity on the determination of $c_{3}$ from $\tau^{-} \rightarrow \eta \pi^{-} \pi^{0} \nu_{\tau}$ decays [89]. We will take $c_{3}=0.007_{-0.012}^{+0.020}$ according to the determinations by Chen et al. in Refs. [90,95,140] (which is also in agreement with the

\footnotetext{
${ }^{19}$ Couplings of operators with two resonance fields are dimensionless $[83,88]$.
} 
most elaborated $e^{+} e^{-} \rightarrow\left(\eta / \pi^{0}\right) \pi^{+} \pi^{-}$fit [92]). Although $c_{4}$ was first evaluated by studying $\sigma\left(e^{+} e^{-} \rightarrow K K \pi\right)$ in Ref. [86], this yielded an inconsistent result for $\tau^{-} \rightarrow$ $K^{-} \gamma \nu_{\tau}$ branching ratio [87], so we will use $c_{4}=-0.0024 \pm$ 0.0006 [95] as the most reliable estimation. In view of all these results, we will take $\left|c_{i}\right| \lesssim 0.015$ as a reasonable estimate, which is translated to $\left|\kappa_{i}^{V}\right| \lesssim 0.025 \mathrm{GeV}^{-1}$. Since there is not enough information on $\kappa_{i}^{A}$, we will take $\left|\kappa_{i}^{A}\right| \sim\left|\kappa_{i}^{V}\right| \lesssim 0.025 \mathrm{GeV}^{-1}$. We will see in the following sections that the observables that we consider and the IB corrections for $a_{\mu}^{\mathrm{HVP},\left.\mathrm{LO}\right|_{\pi \pi}}$ depend mostly on the $\kappa_{i}^{V}$ couplings (besides $F_{V}, G_{V}$, and $F_{A}$ ); for this reason, we perform a global fit to better bind these couplings (see Appendix A). ${ }^{20}$

We turn now to the remaining couplings. We will employ $d_{2}=0.08 \pm 0.08$, which has been determined simultaneously with $c_{3}[90,92,95,140]$. For $d_{4}$, we will assume $\left|d_{4}\right|<0.15$, or in terms of $\kappa_{i}^{V V}$, we get $\left|\kappa_{i}^{V V}\right| \lesssim 0.1$. Again, we will adopt $\left|\kappa_{i}^{V A}\right| \sim\left|\kappa_{i}^{V V}\right| \lesssim 0.1$, which agrees with the prediction $\kappa_{5}^{V A} \sim-0.14$ in Eq. (17).

Using only operators contributing to the $\mathcal{O}\left(p^{4}\right)$ LECs, we have the consistent set for two-point Green's functions (11). However, including operators which start contributing at $\mathcal{O}\left(p^{6}\right)$, we shall use the relations for two- and three-point Green's functions [Eq. (10) and Eqs. (12) to (18)]. In particular, $F_{V}=\sqrt{3} F$, which implies (via (10)) $G_{V}=$ $F / \sqrt{3}$ and $F_{A}=\sqrt{2} F$. Therefore, we will also be showing the Cirigliano et al. results [62] with the latter set of constraints (inconsistent for two-point Green's functions) so that the impact of the change of $F_{V}, F_{A}$, and $G_{V}$ between these two cases is appreciated.

We will refer to the original [62] constraints (11) as $F_{V}=\sqrt{2} F$ and by $F_{V}=\sqrt{3} F$ to their consistent set of values $\left(F_{V}=\sqrt{3} F, G_{V}=F / \sqrt{3}, F_{A}=\sqrt{2} F\right)$ up to threepoint Green's functions. In this last way, we stress that the consistent set of SD constraints in both parity sectors [81,83,88,94] determines the $F_{V}=\sqrt{3} F$ relations (among many others, reviewed in this section).

\section{RADIATIVE CORRECTIONS FOR HADRONIC VACUUM POLARIZATION}

The four-body differential decay width is given by $[62]^{21}$

$$
\begin{aligned}
d \Gamma= & \frac{(2 \pi)^{4}}{2 m_{\tau}} \overline{|\mathcal{M}|^{2}} \delta^{4}\left(P-p_{-}-p_{0}-k-q\right) \\
& \times \frac{d^{3} p_{-}}{(2 \pi)^{3} 2 E_{-}} \frac{d^{3} p_{0}}{(2 \pi)^{3} 2 E_{0}} \frac{d^{3} q}{(2 \pi)^{3} 2 E_{\nu}} \frac{d^{3} k}{(2 \pi)^{3} 2 E_{\gamma}},
\end{aligned}
$$

\footnotetext{
${ }^{20}$ The results obtained assuming $\left|\kappa_{i}^{V}\right| \lesssim 0.025 \mathrm{GeV}^{-1}$ can be found at https://arxiv.org/abs/2007.11019v1. While both results agree remarkably, the errors are reduced in the current procedure.
}

and using the relation $\frac{d^{3} p_{-}}{2 E_{-}} \frac{d^{3} p_{0}}{2 E_{0}}=\frac{\pi^{2}}{4 m_{\tau}^{2}} d s d u d x$ and integrating over the 3 -momentum of the photon and neutrino, ${ }^{22}$ we get

$$
\begin{aligned}
d \Gamma= & \frac{1}{32(2 \pi)^{6} m_{\tau}^{3}}\left[\int \frac{d^{3} q}{2 E_{\nu}} \frac{d^{3} k}{2 E_{\gamma}} \overline{|\mathcal{M}|^{2}} \delta^{4}\left(P-p_{-}-p_{0}-k-q\right)\right] \\
& \times d s d u d x
\end{aligned}
$$

working at leading order in the Low expansion and in the isospin limit $m_{u}=m_{d}$, and we have

$$
\mathcal{M}=e \epsilon^{* \mu}(k) \mathcal{M}_{\pi \pi}^{(0)}\left(\frac{p_{-\mu}}{p_{-} \cdot k}-\frac{P_{\mu}}{P \cdot k}\right)+\mathcal{O}\left(k^{0}\right),
$$

where $\mathcal{M}_{\pi \pi}^{(0)}=G_{F} V_{u d}^{*} \sqrt{S_{\mathrm{EW}}} f_{+}(s)\left(p_{-}-p_{0}\right)_{\nu} \bar{u}(q) \gamma^{\nu}\left(1-\gamma_{5}\right) \times$ $u(P)$ is the amplitude at leading order for the nonradiative decay that includes the SD electroweak radiative corrections $\left(S_{\mathrm{EW}}\right)$. At $\mathcal{O}\left(k^{-1}\right)$, the amplitude for the radiative decay is proportional to the amplitude of the nonradiative decay according to Low's theorem [111].

The unpolarized spin-averaged squared amplitude is given by

$$
\begin{aligned}
\overline{|\mathcal{M}|^{2}}= & 4 \pi \alpha \overline{\left|\mathcal{M}_{\pi \pi}^{(0)}\right|^{2}} \sum_{\gamma} \epsilon^{* \mu}(k) \epsilon^{\nu}(k) \\
& \times\left(\frac{p_{-\mu}}{p_{-} \cdot k+\frac{1}{2} M_{\gamma}^{2}}-\frac{P_{\mu}}{P \cdot k-\frac{1}{2} M_{\gamma}^{2}}\right) \\
& \times\left(\frac{p_{-\nu}}{p_{-} \cdot k+\frac{1}{2} M_{\gamma}^{2}}-\frac{P_{\nu}}{P \cdot k-\frac{1}{2} M_{\gamma}^{2}}\right)+\mathcal{O}\left(k^{-1}\right),
\end{aligned}
$$

using the relation $\sum_{\gamma} \epsilon^{* \mu}(k) \epsilon^{\nu}(k)=-g^{\mu \nu}$ and massive photons $\left(k^{\mu} k_{\mu}=M_{\gamma}^{2}\right)$. The sum over photon polarizations should include the longitudinal part, since our photon has mass and the amplitude is no longer gauge invariant. We do not take into account this contribution because it will vanish in the limit $M_{\gamma} \rightarrow 0$.

Thus, Eq. (24) becomes

$$
\begin{aligned}
\overline{|\mathcal{M}|^{2}}= & 4 \pi \alpha \overline{\left|\mathcal{M}_{\pi \pi}^{(0)}\right|^{2}}\left(\frac{2 P \cdot p_{-}}{\left(p_{-} \cdot k+\frac{1}{2} M_{\gamma}^{2}\right)\left(P \cdot k-\frac{1}{2} M_{\gamma}^{2}\right)}\right. \\
& \left.-\frac{m_{\pi}^{2}}{\left(p_{-} \cdot k+\frac{1}{2} M_{\gamma}^{2}\right)^{2}}-\frac{m_{\tau}^{2}}{\left(P \cdot k-\frac{1}{2} M_{\gamma}^{2}\right)^{2}}\right) \\
& +\mathcal{O}\left(k^{-1}\right),
\end{aligned}
$$

\footnotetext{
${ }^{21}$ Although the analytical results in this section were presented in the quoted reference, we include them here, given their importance in the evaluation of the relevant IB corrections, and take advantage to add a few explanations to previous discussions of this subject $[62,66]$.

${ }^{22}$ The kinematics for these decays are in Appendix B.
} 
where

$\overline{\left|\mathcal{M}_{\pi \pi}^{(0)}\right|^{2}}=4 G_{F}^{2}\left|V_{u d}\right|^{2} S_{\mathrm{EW}}\left|f_{+}(s)\right|^{2}(D(s, u)+\mathcal{O}(k))$,

with $D(s, u)=\frac{1}{2} m_{\tau}^{2}\left(m_{\tau}^{2}-s\right)+2 m_{\pi}^{4}-2 u\left(m_{\tau}^{2}-s+2 m_{\pi}^{2}\right)+2 u^{2}$. Equation (25) does not contribute at $\mathcal{O}\left(k^{-1}\right)$, and these terms are canceled out by those in Eq. (26) according to the Burnett-Kroll theorem [141].

Replacing Eqs. (25) and (26) in Eq. (22), we get

$$
\begin{aligned}
d \Gamma= & \frac{\alpha G_{F}^{2}\left|V_{u d}\right|^{2} S_{\mathrm{EW}}}{4(2 \pi)^{4} m_{\tau}^{3}}\left|f_{+}(s)\right|^{2} D(s, u) \\
& \times\left(2 P \cdot p_{-} I_{11}(s, u, x)-m_{\pi}^{2} I_{02}(s, u, x)-m_{\tau}^{2} I_{20}(s, u, x)\right) \\
& \times d s d u d x+\mathcal{O}\left(k^{0}\right) ;
\end{aligned}
$$

the $I_{m n}(s, u, x)$ is defined as

$I_{m n}(s, u, x)=\frac{1}{2 \pi} \int \frac{d^{3} q}{2 E_{\nu}} \frac{d^{3} k}{2 E_{\gamma}} \frac{\delta^{4}\left(P-p_{-}-p_{0}-k-q\right)}{\left(P \cdot k-\frac{1}{2} M_{\gamma}^{2}\right)^{m}\left(p_{-} \cdot k+\frac{1}{2} M_{\gamma}^{2}\right)^{n}}$,

performing an integration over $x$; and we can split the decay width according to the integration region

$$
\frac{d^{2} \Gamma}{d s d u}=\left.\frac{d^{2} \Gamma}{d s d u}\right|_{\mathcal{D}^{I I I}}+\left.\frac{d^{2} \Gamma}{d s d u}\right|_{\mathcal{D}^{I V / I I I}}+\mathcal{O}\left(k^{0}\right),
$$

where

$$
\begin{aligned}
\left.\frac{d^{2} \Gamma}{d s d u}\right|_{\mathcal{D}^{I I I}}= & \frac{\alpha G_{F}^{2}\left|V_{u d}\right|^{2} S_{\mathrm{EW}}}{4(2 \pi)^{4} m_{\tau}^{3}}\left|f_{+}(s)\right|^{2} D(s, u) \\
& \times\left(J_{11}\left(s, u, M_{\gamma}\right)+J_{02}\left(s, u, M_{\gamma}\right)+J_{20}\left(s, u, M_{\gamma}\right)\right)
\end{aligned}
$$

and

$$
\begin{aligned}
\left.\frac{d^{2} \Gamma}{d s d u}\right|_{\mathcal{D}^{I V / I I I}}= & \frac{\alpha G_{F}^{2}\left|V_{u d}\right|^{2} S_{\mathrm{EW}}}{4(2 \pi)^{4} m_{\tau}^{3}}\left|f_{+}(s)\right|^{2} D(s, u) \\
& \times\left(K_{11}(s, u)+K_{02}(s, u)+K_{20}(s, u)\right),
\end{aligned}
$$

with

$$
\begin{gathered}
J_{m n}\left(s, u, M_{\gamma}\right)=c_{m n} \int_{M_{\gamma}^{2}}^{x_{+}(s, u)} d x I_{m n}(s, u, x), \\
K_{m n}(s, u)=c_{m n} \int_{x_{-}(s, u)}^{x_{+}(s, u)} d x I_{m n}(s, u, x),
\end{gathered}
$$

and

$$
c_{m n}= \begin{cases}2 P \cdot p_{-} & m=n=1, \\ -m_{\tau}^{2} & m=2, n=0, \\ -m_{\pi^{-}}^{2} & m=0, n=2 .\end{cases}
$$

Equation (28) is an invariant, so we can evaluate it in any reference frame in order to simplify the integration, and working in the $\gamma-\nu_{\tau}$ center of mass, we have

$$
\begin{aligned}
I_{m n}(s, u)= & \frac{1}{2^{3}(2 \pi)} \int \frac{x-M_{\gamma}^{2}}{x\left(P \cdot k-\frac{1}{2} M_{\gamma}^{2}\right)^{m}\left(p_{-} \cdot k+\frac{1}{2} M_{\gamma}^{2}\right)^{n}} \\
& \times d \cos \theta_{\nu} d \phi_{-} .
\end{aligned}
$$

Integrating this equation over $x$ in $\mathcal{D}_{I V / I I I}$ and $\mathcal{D}_{I I I}$, as in Refs. [62,142], we get $\left[\operatorname{Li}_{2}(x)=-\int_{0}^{1} \frac{d t}{t} \log (1-x t)\right]$

$$
\begin{gathered}
J_{11}(s, u)=\log \left(\frac{2 x_{+}(s, u) \bar{\gamma}}{M_{\gamma}}\right) \frac{1}{\bar{\beta}} \log \left(\frac{1+\bar{\beta}}{1-\bar{\beta}}\right) \\
+\frac{1}{\bar{\beta}}\left(L i_{2}\left(1 / Y_{2}\right)-L i_{2}\left(Y_{1}\right)+\log ^{2}\left(-1 / Y_{2}\right) / 4\right. \\
\left.-\log ^{2}\left(-1 / Y_{1}\right) / 4\right) \\
J_{20}(s, u)=\log \left(\frac{M_{\gamma}\left(m_{\tau}^{2}-s\right)}{m_{\tau} x_{+}(s, u)}\right) \\
J_{02}(s, u)=\log \left(\frac{M_{\gamma}\left(m_{\tau}^{2}+m_{\pi^{0}}^{2}-s-u\right)}{m_{\pi}^{-} x_{+}(s, u)}\right) \\
K_{20}(s, u)=K_{0,2}(s, u)=\log \left(\frac{x_{-}(s, u)}{x_{+}(s, u)}\right)
\end{gathered}
$$

where the expressions in Eq. (36) are given by

$$
Y_{1,2}=\frac{1-2 \bar{\alpha} \pm \sqrt{(1-2 \bar{\alpha})^{2}-\left(1-\bar{\beta}^{2}\right)}}{1+\bar{\beta}},
$$

with

$$
\begin{aligned}
& \bar{\alpha}=\frac{\left(m_{\tau}^{2}-s\right)\left(m_{\tau}^{2}+m_{\pi^{0}}^{2}-s-u\right)}{\left(m_{\pi^{-}}^{2}+m_{\tau}^{2}-u\right)} \cdot \frac{\lambda\left(u, m_{\pi^{-}}^{2}, m_{\tau}^{2}\right)}{2 \bar{\delta}}, \\
& \bar{\beta}=-\frac{\sqrt{\lambda\left(u, m_{\pi^{-}}^{2}, m_{\tau}^{2}\right)}}{m_{\pi^{-}}^{2}+m_{\tau}^{2}-u}
\end{aligned}
$$

$\bar{\gamma}=\frac{\sqrt{\lambda\left(u, m_{\pi^{-}}^{2}, m_{\tau}^{2}\right)}}{2 \sqrt{\delta}}$,

$$
\begin{aligned}
\bar{\delta}= & -m_{\pi^{0}}^{4} m_{\tau}^{2}+m_{\pi^{-}}^{2}\left(m_{\tau}^{2}-s\right)\left(m_{\pi^{0}}^{2}-u\right)-s u\left(-m_{\tau}^{2}+s+u\right) \\
& +m_{\pi^{0}}^{2}\left(-m_{\tau}^{4}+s u+m_{\tau}^{2} s+m_{\tau}^{2} u\right) .
\end{aligned}
$$

Experimentally, it is impossible to measure the full photon spectrum because of acceptances, efficiencies, 
and cuts. For this reason, we need to calculate the inclusive decay width, since we cannot distinguish the radiative decay from the nonradiative decay for low-energy (or collinear) photons.

For the nonradiative decay, we have

$\frac{d^{2} \Gamma}{d s d u}=\frac{G_{F}^{2}\left|V_{u d}\right|^{2} S_{\mathrm{EW}}}{64 \pi^{3} m_{\tau}^{3}}\left|f_{+}(s)\right|^{2}\left(1+f_{\text {loop }}^{\mathrm{elm}}\left(u, M_{\gamma}\right)\right)^{2} D(s, u)$,

which includes isospin violation and photonic corrections according to Ref. [61], where $f_{\text {loop }}^{\mathrm{elm}}\left(u, M_{\gamma}\right)$ is given by

$$
\begin{aligned}
f_{\text {loop }}^{\operatorname{elm}}\left(u, M_{\gamma}\right)= & \frac{\alpha}{4 \pi}\left(\left(u-m_{\pi}^{2}\right) \mathcal{A}(u)+\left(u-m_{\pi}^{2}-m_{\tau}^{2}\right) \mathcal{B}(u)\right. \\
& \left.+2\left(m_{\pi}^{2}+m_{\tau}^{2}-u\right) \mathcal{C}\left(u, M_{\gamma}\right)+2 \log \frac{m_{\pi} m_{\tau}}{M_{\gamma}^{2}}\right),
\end{aligned}
$$

with

$$
\begin{aligned}
& \mathcal{A}(u)=\frac{1}{u}\left(-\frac{1}{2} \log r_{\tau}+\frac{2-y_{\tau}}{\sqrt{r_{\tau}}} \frac{x_{\tau}}{1-x_{\tau}^{2}} \log x_{\tau}\right), \\
& \mathcal{B}(u)=\frac{1}{u}\left(\frac{1}{2} \log r_{\tau}+\frac{2 r_{\tau}-y_{\tau}}{\sqrt{r_{\tau}}} \frac{x_{\tau}}{1-x_{\tau}^{2}} \log x_{\tau}\right),
\end{aligned}
$$

$\mathcal{C}\left(u, M_{\gamma}\right)=\frac{1}{m_{\tau} m_{\pi}} \frac{x_{\tau}}{1-x_{\tau}^{2}}\left(-\frac{1}{2} \log ^{2} x_{\tau}+2 \log x_{\tau} \log \left(1-x_{\tau}^{2}\right)\right.$

$$
\begin{aligned}
& -\frac{\pi^{2}}{6}+\frac{1}{8} \log ^{2} r_{\tau} \\
& +L i_{2}\left(x_{\tau}^{2}\right)+L i_{2}\left(1-\frac{x_{\tau}}{\sqrt{r_{\tau}}}\right) \\
& \left.+L i_{2}\left(1-x_{\tau} \sqrt{r_{\tau}}\right)-\log x_{\tau} \log \frac{M_{\gamma}^{2}}{m_{\tau} m_{\pi}}\right),
\end{aligned}
$$

in terms of the variables

$r_{\tau}=\frac{m_{\tau}^{2}}{m_{\pi}^{2}}, \quad y_{\tau}=1+r_{\tau}-\frac{u}{m_{\pi}^{2}}, \quad x_{\tau}=\frac{1}{2 \sqrt{r_{\tau}}}\left(y_{\tau}-\sqrt{y_{\tau}^{2}-4 r_{\tau}}\right)$

Thus, the inclusive decay width is

$\left.\frac{d^{2} \Gamma}{d s d u}\right|_{\pi \pi(\gamma)}=\frac{G_{F}^{2}\left|V_{u d}\right|^{2} S_{\mathrm{EW}}}{64 \pi^{3} m_{\tau}^{3}}\left|f_{+}(s)\right|^{2} D(s, u) \Delta(s, u)$,

where

$$
\Delta(s, u)=1+2 f_{\text {loop }}^{\text {elm }}\left(u, M_{\gamma}\right)+g_{\text {rad }}\left(s, u, M_{\gamma}\right) .
$$

In the previous expression, we neglected the quadratic term for $f_{\text {loop }}^{\text {elm }}\left(u, M_{\gamma}\right)$, and

$$
g_{\text {rad }}\left(s, u, M_{\gamma}\right)=g_{\text {brems }}\left(s, u, M_{\gamma}\right)+g_{\text {rest }}(s, u) \text {, }
$$

with

$$
\begin{aligned}
g_{\text {brems }}\left(s, u, M_{\gamma}\right)= & \frac{\alpha}{\pi}\left(J_{11}\left(s, u, M_{\gamma}\right)+J_{20}\left(s, u, M_{\gamma}\right)\right. \\
& \left.+J_{02}\left(s, u, M_{\gamma}\right)\right),
\end{aligned}
$$

$g_{\mathrm{rest}}(s, u)=\frac{\alpha}{\pi}\left(K_{11}(s, u)+K_{20}(s, u)+K_{02}(s, u)\right)$.

Integrating Eq. (45) over $u$ and using

$\int_{u_{-}(s)}^{u_{+}(s)} D(s, u) d u=\frac{m_{\tau}^{6}}{6}\left(1-\frac{s}{m_{\tau}^{2}}\right)^{2}\left(1-\frac{4 m_{\pi}^{2}}{s}\right)^{3 / 2}\left(1+\frac{2 s}{m_{\tau}^{2}}\right)$,

we have

$$
\begin{aligned}
\left.\frac{d \Gamma}{d s}\right|_{\pi \pi(\gamma)}= & \frac{G_{F}^{2}\left|V_{u d}\right|^{2} m_{\tau}^{3} S_{\mathrm{EW}}}{384 \pi^{3}}\left|f_{+}(s)\right|^{2}\left(1-\frac{s}{m_{\tau}^{2}}\right)^{2} \\
& \times\left(1-\frac{4 m_{\pi}^{2}}{s}\right)^{3 / 2}\left(1+\frac{2 s}{m_{\tau}^{2}}\right) G_{\mathrm{EM}}(s)
\end{aligned}
$$

for this, we follow the same notation as in Ref. [62]:

$$
G_{\mathrm{EM}}(s)=\frac{\int_{\mathcal{R}^{I V}} D(s, u) \Delta(s, u) d u}{\int_{u_{-}(s)}^{u_{+}(s)} D(s, u) d u}
$$

We can split the electromagnetic correction factor $\left[G_{\mathrm{EM}}(s)\right]$ in two parts, $G_{\mathrm{EM}}^{(0)}(s)$ and $G_{\mathrm{EM}}^{\text {rest }}(s)$; the first one corresponds to taking $g_{\text {rest }}(s, u) \rightarrow 0$, and the second one is the remainder of $G_{\mathrm{EM}}(s)$,

$$
\begin{aligned}
& G_{\mathrm{EM}}^{(0)}(s) \\
& =\frac{\int_{\mathcal{R}^{I I I}} D(s, u)\left(1+2 f_{\text {loop }}^{\text {elm }}\left(u, M_{\gamma}\right)+g_{\text {brems }}\left(s, u, M_{\gamma}\right)\right) d u}{\int_{u_{-}(s)}^{u_{+}(s)} D(s, u) d u},
\end{aligned}
$$

$$
G_{\mathrm{EM}}^{\mathrm{rest}}(s)=\frac{\int_{\mathcal{R}^{I V / I I I}} D(s, u) g^{\mathrm{rest}}(s, u) d u}{\int_{u_{-}(s)}^{u_{+}(s)} D(s, u) d u} .
$$

In Eq. (51a), the term $2 f_{\text {loop }}^{\text {elm }}\left(u, M_{\gamma}\right)+g_{\text {brems }}\left(s, u, M_{\gamma}\right)$ is finite when $M_{\gamma} \rightarrow 0$, 

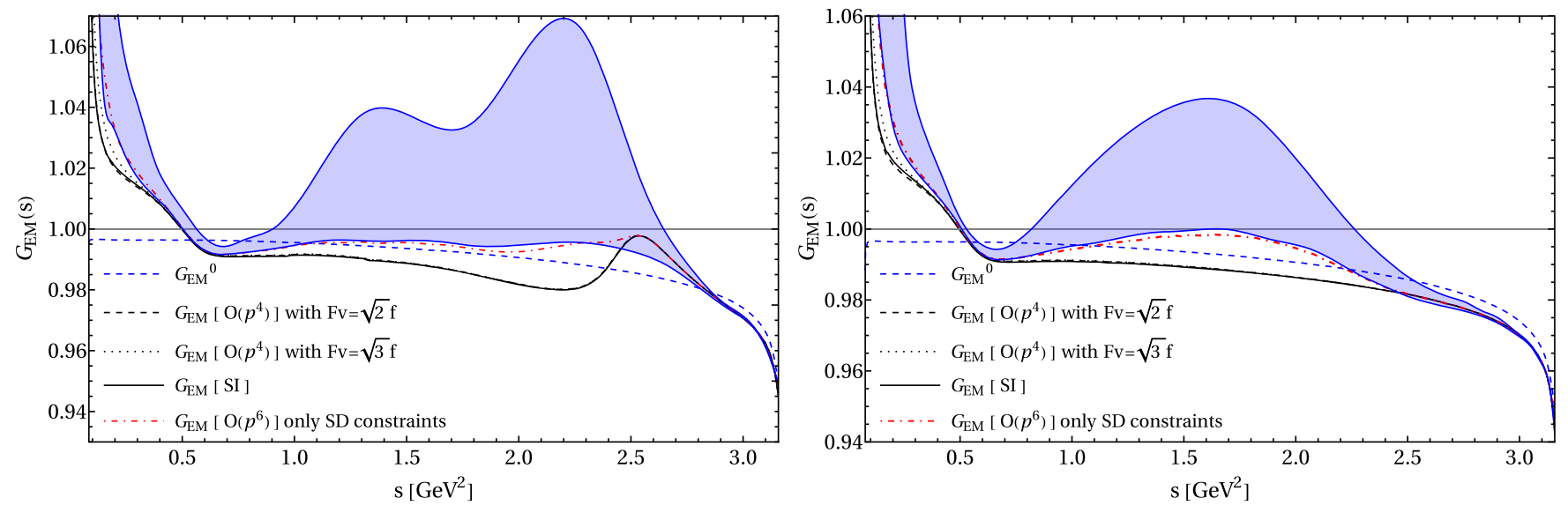

FIG. 8. Correction function $G_{\mathrm{EM}}^{(0)}(s)$ in Eq. (51a) (blue dashed line). The solid line shows the $G_{\mathrm{EM}}(s)$ function neglecting the structuredependent part (SI), i.e., by taking $v_{1}=v_{2}=v_{3}=v_{4}=a_{1}=a_{2}=a_{3}=a_{4}=0$; the dashed and dotted lines are the $\mathcal{O}\left(p^{4}\right) G_{\mathrm{EM}}(s)$ function (with either $F_{V}=\sqrt{2} F$ or $F_{V}=\sqrt{3} F$ constraints). The blue shaded region is the full $\mathcal{O}\left(p^{6}\right)$ contribution, including (overestimated) uncertainties. The left-hand side plot corresponds to the dispersive parametrization [124], while the right-hand side corresponds to the Guerrero-Pich parametrization [143] of the form factor (the latter was used in Ref. [62]).

$$
\begin{aligned}
2 f_{\text {loop }}^{\text {elm }} & \left(u, M_{\gamma}\right)+g_{\text {brems }}\left(s, u, M_{\gamma}\right) \\
= & \frac{\alpha}{4 \pi}\left(\left(u-m_{\pi}^{2}\right) \mathcal{A}(u)+\left(u-m_{\pi}^{2}-m_{\tau}^{2}\right) \mathcal{B}(u)\right. \\
& \left.+2\left(m_{\pi}^{2}+m_{\tau}^{2}-u\right) \mathcal{C}(u)\right) \\
& +\frac{\alpha}{\pi}\left(J_{11}(s, u)+J_{20}(s, u)+J_{02}(s, u)\right) .
\end{aligned}
$$

In this limit, we have

$$
\begin{aligned}
& \mathcal{C}(u)= \frac{1}{m_{\tau} m_{\pi}} \frac{x_{\tau}}{1-x_{\tau}^{2}}\left(-\frac{1}{2} \log ^{2} x_{\tau}+2 \log x_{\tau} \log \left(1-x_{\tau}^{2}\right)\right. \\
&-\frac{\pi^{2}}{6}+\frac{1}{8} \log ^{2} r_{\tau}+L i_{2}\left(x_{\tau}^{2}\right)+L i_{2}\left(1-\frac{x_{\tau}}{\sqrt{r_{\tau}}}\right) \\
&+\left.L i_{2}\left(1-x_{\tau} \sqrt{r_{\tau}}\right)\right), \\
& J_{11}(s, u)= \frac{1}{2} \log \left(\frac{4 x_{+}^{2}(s, u) \bar{\gamma}^{2}}{m_{\pi} m_{\tau}}\right) \frac{1}{\bar{\beta}} \log \left(\frac{1+\bar{\beta}}{1-\bar{\beta}}\right) \\
&+\frac{1}{\bar{\beta}}\left(L i_{2}\left(1 / Y_{2}\right)-L i_{2}\left(Y_{1}\right)\right. \\
&\left.+\log ^{2}\left(-1 / Y_{2}\right) / 4-\log ^{2}\left(-1 / Y_{1}\right) / 4\right), \\
& J_{20}(s, u)=\log \left(\frac{m_{\tau}^{2}-s}{x_{+}(s, u)}\right), \\
& J_{02}(s, u)=\log \left(\frac{m_{\tau}^{2}+m_{\pi}^{2}-s-u}{x_{+}(s, u)}\right)
\end{aligned}
$$

where $x_{+}(s, u)$ is defined in Eq. (B19).

The leading Low approximation for $G_{\mathrm{EM}}^{(0)}(s)$ is plotted in Fig. 8. This function has two poles, one at $s=4 m_{\pi}^{2}$ and the other at $s=m_{\tau}^{2}$.
We will use the same conventions as Ref. [62], so we denote as "complete bremsstrahlung" the amplitude where the structure-dependent part vanishes, i.e., $v_{1}=v_{2}=v_{3}=v_{4}=a_{1}=a_{2}=a_{3}=a_{4}=0$. For convenience, we will refer in the following simply as $\mathcal{O}\left(p^{4}\right)$ and $\mathcal{O}\left(p^{6}\right)$ to the contributions from $R \chi T$ including operators that contribute up to $\mathcal{O}\left(p^{4}\right)$ and up to $\mathcal{O}\left(p^{6}\right)$ chiral LECs, respectively. ${ }^{23}$

In $G_{\mathrm{EM}}(s)$, the difference between using the $F_{V}=\sqrt{2} F$ or $F_{V}=\sqrt{3} F$ constraints at $\mathcal{O}\left(p^{4}\right)$ is only appreciated for $s \lesssim 0.35 \mathrm{GeV}^{2}$, with the latter set producing the largest deviation with respect to the SI result (Fig. 8). It is important to note that - as put forward in Ref. [62]—with $F_{V}=\sqrt{2} F$ constraints (those consistent for two-point Green's functions) the impact of the structure-dependent corrections on $G_{\mathrm{EM}}(s)$ is negligible and the evaluation with SI gives already an excellent approximation. On the contrary, we find that using the $F_{V}=\sqrt{3} F$ set this is no longer true, which will increase the $G_{\mathrm{EM}}(s)$ correction in $a_{\mu}^{\mathrm{HVP},\left.\mathrm{LO}\right|_{\pi \pi}}$ using $\tau$ data [even before adding the $\mathcal{O}\left(p^{6}\right)$ contributions].

In Fig. 8, several contributions to the $G_{\mathrm{EM}}(s)$ function are shown: the $G_{\mathrm{EM}}^{(0)}$ part by a dashed blue line and the complete bremsstrahlung (SI) contribution with a solid black line. The full amplitude including all $R \chi T$ operators which contribute at $\mathcal{O}\left(p^{4}\right)\left[\mathcal{O}\left(p^{6}\right)\right]$ is represented by black dashed/dotted (red dash-dotted) lines in Fig. 8. For the $\mathcal{O}\left(p^{4}\right)$ contribution, we distinguish between using $F_{V}=$ $\sqrt{2} F\left(F_{V}=\sqrt{3} F\right)$, represented by dashed (dotted) lines. Compared to previous results $[61,62,65,66]$, we note the

\footnotetext{
${ }^{23}$ The different SD constraints applying in each case were discussed at length in Sec. IIE.
} 
appearance of a bump near the end of the phase space on $G_{\mathrm{EM}}(s)$ due to the inclusion of the $\rho(1450)$ and the $\rho(1700)$ resonances in the dispersive representation of the vector form factor $[124,125]$. The blue band in Fig. 8 shows the uncertainty of the $\mathcal{O}\left(p^{6}\right)$ contribution, evaluated according to that on the couplings which were determined phenomenologically or estimated from chiral counting in Sec. II E (see also Appendix A). ${ }^{24}$ While the central values of the $\mathcal{O}\left(p^{6}\right)$ corrections change mildly the results obtained at $\mathcal{O}\left(p^{4}\right),{ }^{25}$ their huge uncertainty band suggests that our estimate of the $R \chi T$ couplings which start contributing at $\mathcal{O}\left(p^{6}\right)$ was very conservative (one naively expects and approximate $1 / N_{C}$ uncertainty for a large- $N_{C}$ expansion ${ }^{26}$ ). Lacking a better way for this estimation, we consider this uncertainty band as a conservative upper limit on the corresponding uncertainties. Therefore, our error bands at $\mathcal{O}\left(p^{6}\right)$ should be regarded accordingly in the following. On the contrary, the small modification induced by those $\mathcal{O}\left(p^{6}\right)$ couplings fixed by SD constraints (with all remaining ones vanishing) with respect to the $\mathcal{O}\left(p^{4}\right)$ [62] results suggests that the difference between those is a realistic estimate of the missing subdominant terms in Ref. $[62]^{27}$ and will be given as such in the remainder of the paper.

\section{A. Radiative decay}

The differential decay width [142] is given by

$d \Gamma=\frac{\lambda^{1 / 2}\left(s, m_{\pi^{0}}^{2}, m_{\pi^{-}}^{2}\right)}{2(4 \pi)^{6} m_{\tau}^{2} s} \overline{|\mathcal{M}|^{2}} d E_{\gamma} d x d s d \cos \theta_{-} d \phi_{-}$,

where $\overline{|\mathcal{M}|^{2}}$ is the unpolarized spin-averaged squared amplitude that corresponds to the $\tau^{-} \rightarrow \pi^{-} \pi^{0} \gamma \nu_{\tau}$ decays and $E_{\gamma}$ is the photon energy in the $\tau$ rest frame. It is not worth it to quote here the full analytical expression for $\overline{|\mathcal{M}|^{2}}$.

\footnotetext{
${ }^{24}$ These were varied assuming Gaussian errors, and the band was generated so as to cover all data points obtained in 100 spectrum simulations. Results were stable upon increasing statistics. The corresponding blue bands were obtained similarly in Figs. 12-14.

${ }^{25}$ This is reasonable, since SI is basically unchanged by the $\mathcal{O}\left(p^{4}\right)$ contributions.

${ }^{26}$ This rough estimate of the parametric uncertainty is supported by the computation of $\chi P T$ LECs including such corrections (see, e.g., Refs. $[119,121,123]$ ). We note that in this work resonance widths (dominant next-to-leading-order effect in the large- $N_{C}$ expansion for the considered decays) are included. Also, the uncertainty corresponding to including excited resonances (an infinite number of them appears for $N_{C} \rightarrow \infty$ ) was checked to be negligible.

${ }^{27}$ These were not estimated in Ref. [62] as SI was already an excellent approximation to the result up to $\mathcal{O}\left(p^{4}\right)$ (using the $F_{V}=\sqrt{2} F$ set).
}

For these decays, we have the integration region

$$
\begin{gathered}
\mathcal{D}=\left\{E_{\gamma}^{\min } \leq E_{\gamma} \leq E_{\gamma}^{\max }, x_{\min } \leq x \leq x_{\max }, s_{\min } \leq s \leq s_{\max }\right. \\
\left.-1 \leq \cos \theta_{-} \leq+1,0 \leq \phi_{-} \leq 2 \pi\right\}
\end{gathered}
$$

with boundaries

$$
\begin{aligned}
& \frac{\left(m_{\tau}^{2}-s+x\right)}{4 m_{\tau}^{2}}-\frac{\lambda^{1 / 2}\left(s, x, m_{\tau}^{2}\right)}{4 m_{\tau}} \\
& \quad \leq E_{\gamma}(s, x) \leq \frac{\left(m_{\tau}^{2}-s+x\right)}{4 m_{\tau}}+\frac{\lambda^{1 / 2}\left(s, x, m_{\tau}^{2}\right)}{4 m_{\tau}}, \\
& 4 m_{\pi}^{2} \leq s(x) \leq\left(m_{\tau}-\sqrt{x}\right)^{2}, \\
& 0 \leq x \leq\left(m_{\tau}-2 m_{\pi}\right)^{2}
\end{aligned}
$$

or interchanging the last two limits,

$$
0 \leq x(s) \leq\left(m_{\tau}-\sqrt{s}\right)^{2}, \quad 4 m_{\pi}^{2} \leq s \leq m_{\tau}^{2} .
$$

There are other ways to write these,

$$
\begin{aligned}
4 m_{\pi}^{2} & \leq s\left(x, E_{\gamma}\right) \leq \frac{\left(m_{\tau}-2 E_{\gamma}\right)\left(2 m_{\tau} E_{\gamma}-x\right)}{2 E_{\gamma}} \\
0 & \leq x\left(E_{\gamma}\right) \leq \frac{2 E_{\gamma}\left(m_{\tau}^{2}-4 m_{\pi}^{2}-2 m_{\tau} E_{\gamma}\right)}{m_{\tau}-2 E_{\gamma}}, \\
E_{\gamma}^{\text {cut }} & \leq E_{\gamma} \leq \frac{m_{\tau}^{2}-4 m_{\pi}^{2}}{2 m_{\tau}}
\end{aligned}
$$

or exchanging $x \leftrightarrow E_{\gamma}$,

$$
\begin{aligned}
& \frac{\left(m_{\tau}^{2}+x-4 m_{\pi}^{2}\right)}{4 m_{\tau}}-\frac{\lambda^{1 / 2}\left(x, m_{\tau}^{2}, 4 m_{\pi}^{2}\right)}{4 m_{\tau}} \\
& \leq E_{\gamma}(s) \leq \frac{\left(m_{\tau}^{2}+x-4 m_{\pi}^{2}\right)}{4 m_{\tau}}+\frac{\lambda^{1 / 2}\left(x, m_{\tau}^{2}, 4 m_{\pi}^{2}\right)}{4 m_{\tau}}, \\
& 0 \leq x \leq\left(m_{\tau}-2 m_{\pi}\right)^{2}
\end{aligned}
$$

and

$$
\begin{aligned}
0 & \leq x\left(s, E_{\gamma}\right) \leq \frac{2 E_{\gamma}\left(m_{\tau}^{2}-s-2 E_{\gamma} m_{\tau}\right)}{m_{\tau}-2 E_{\gamma}} \\
4 m_{\pi}^{2} & \leq s\left(E_{\gamma}\right) \leq m_{\tau}\left(m_{\tau}-2 E_{\gamma}\right), \\
E_{\gamma}^{\text {cut }} & \leq E_{\gamma} \leq \frac{m_{\tau}^{2}-4 m_{\pi}^{2}}{2 m_{\tau}} .
\end{aligned}
$$

Further, interchanging $s \leftrightarrow E_{\gamma}$, we get 

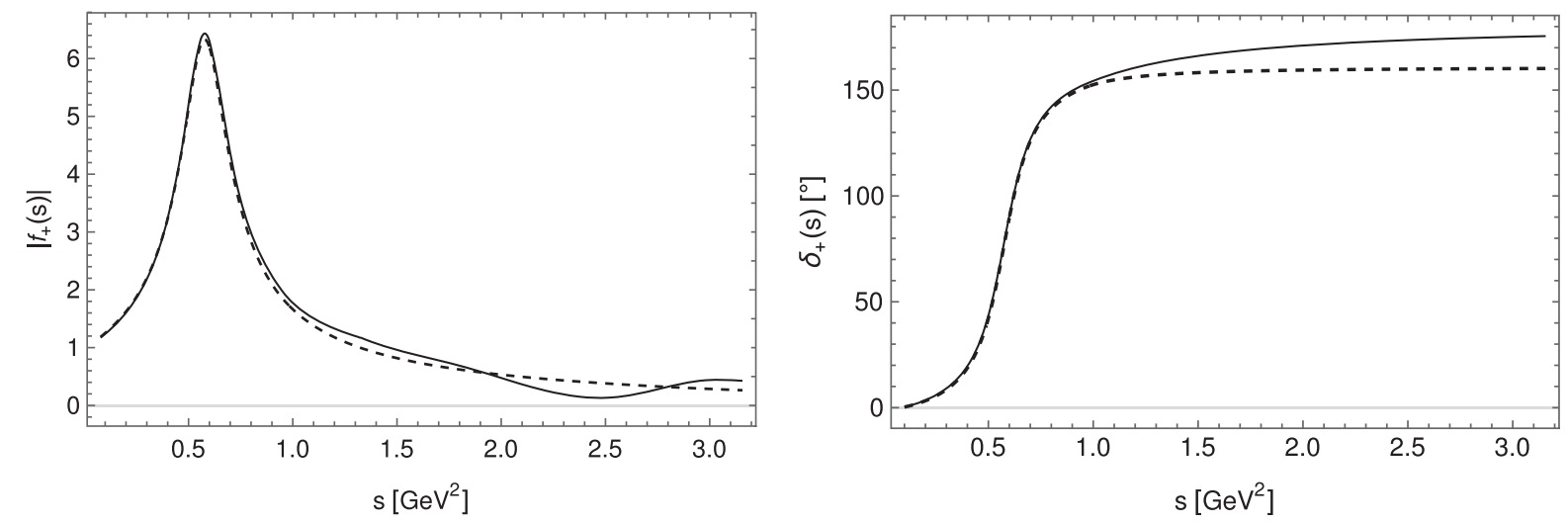

FIG. 9. Modulus and phase of the pion vector form factor, $f_{+}(s)$. The solid line corresponds to the dispersive representation used in Ref. [124], while the dashed line corresponds to the Guerrero-Pich parametrization [143] employed by Ref. [62].

$$
\begin{aligned}
& E_{\gamma}^{\text {cut }} \leq E_{\gamma}(s) \leq \frac{m_{\tau}^{2}-s}{2 m_{\tau}}, \\
& 4 m_{\pi}^{2} \leq s \leq m_{\tau}\left(m_{\tau}-2 E_{\gamma}^{\text {cut }}\right) .
\end{aligned}
$$

We recall that this amplitude has IR divergences due to soft photons, i.e., $E_{\gamma} \rightarrow 0$, which is the same problem with $M_{\gamma} \rightarrow 0$ outlined in the previous section. Correspondingly, the experiment is not able to measure photons with energies smaller than some $E_{\gamma}^{\text {cut }}$ (which is related with the experimental resolution).

Concerning the $\mathcal{O}\left(p^{6}\right)$ contributions, once we employ the relations obtained from the SD behavior of QCD and its OPE, it is seen that observables are basically insensitive (at the percent level of precision) to $\mathcal{O}(1)$ changes of all the couplings but $\kappa_{i}^{V}$ (the $\rho-\omega-\pi$ vertex is described by these couplings), which will saturate the (overestimated) uncertainty of our predictions at this order.

If we integrate Eq. (57) using the limits in Eq. (64) and the dispersive vector form factor $[124,125]$, we get the $\pi^{-} \pi^{0}$ invariant mass distribution, the photon energy distribution, and the branching ratios as a function of $E_{\gamma}^{\text {cut }}$, shown in Figs. 10-14 and summarized in Table II. In these figures, the dot-dashed red line corresponds to taking the limit where all the couplings at $\mathcal{O}\left(p^{6}\right)$ vanish except for those constrained by $\mathrm{SD}$, and the band overestimates the corresponding uncertainties.

As it can be observed from Table II and Fig. 14, the main contribution at $\mathcal{O}\left(p^{4}\right)$ corresponds to the complete bremsstrahlung (SI) amplitude (in agreement with Ref. [62]), and the value for the branching ratio becomes smaller with larger values of $E_{\gamma}^{\text {cut }}$. The values in Table II are slightly different from those reported in Ref. [62]; this effect is mainly due to the parametrization of the pion vector form factor (see Fig. 9). The form factor obtained from the dispersion relation [124] is above the one obtained using the Guerrero-Pich parametrization [143] at $s \simeq M_{\rho}^{2}$, and also the former includes the $\rho(1450)$ and $\rho(1700)$ resonances.

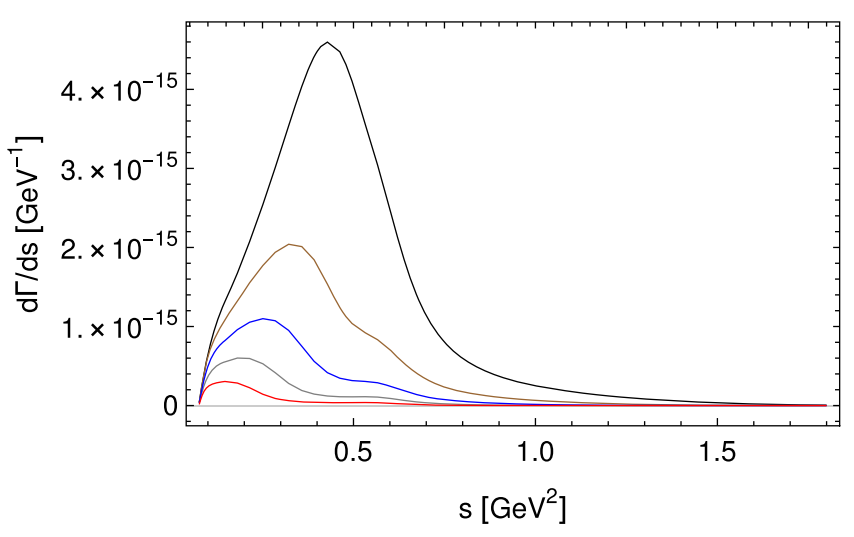

FIG. 10. The $\pi^{-} \pi^{0}$ hadronic invariant mass distribution for the $\tau^{-} \rightarrow \pi^{-} \pi^{0} \gamma \nu_{\tau}$ decays for $E_{\gamma}^{\text {cut }}=100 \mathrm{MeV}$ (black), $E_{\gamma}^{\text {cut }}=$ $200 \mathrm{MeV}$ (brown), $E_{\gamma}^{\text {cut }}=300 \mathrm{MeV}$ (blue), $E_{\gamma}^{\text {cut }}=400 \mathrm{MeV}$ (gray), and $E_{\gamma}^{\text {cut }}=500 \mathrm{MeV}$ (red) using only the bremsstrahlung (SI) contribution.

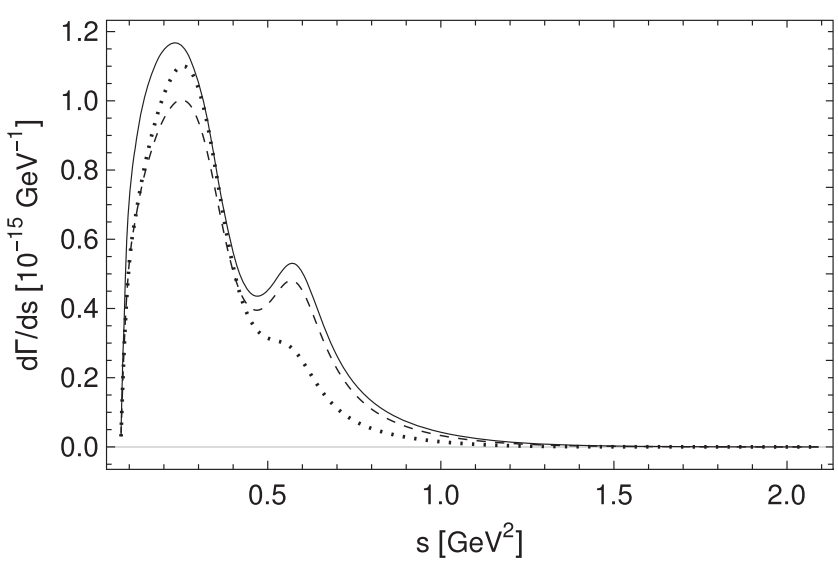

FIG. 11. The $\pi^{-} \pi^{0}$ hadronic invariant mass distributions for $E_{\gamma}^{\text {cut }}=300 \mathrm{MeV}$. The solid and dashed lines represent the $\mathcal{O}\left(p^{4}\right)$ corrections using $F_{V}=\sqrt{3} F$ and $F_{V}=\sqrt{2} F$, respectively. The dotted line stands for the bremsstrahlung contribution (SI). 


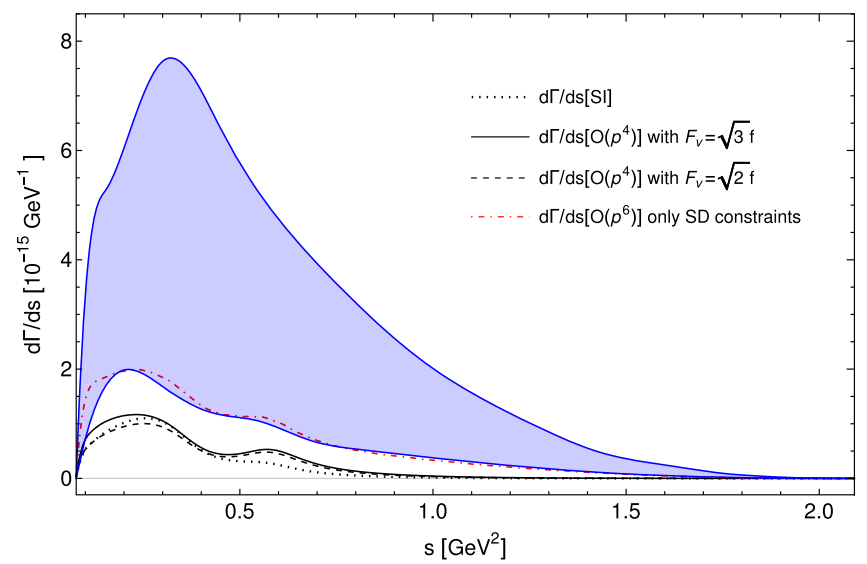

FIG. 12. The $\pi^{-} \pi^{0}$ hadronic invariant mass distributions for $E_{\gamma}^{\text {cut }}=300 \mathrm{MeV}$. The solid and dashed lines represent the $\mathcal{O}\left(p^{4}\right)$ corrections using $F_{V}=\sqrt{3} F$ and $F_{V}=\sqrt{2} F$, respectively. The dotted line represents the bremsstrahlung contribution (SI). The dot-dashed red line corresponds to using only SD constraints at $\mathcal{O}\left(p^{6}\right)$, and the blue shaded region overestimates the corresponding uncertainties.

According to our discussion on error estimation of the $\mathcal{O}\left(p^{4}\right)$ result [including the uncertainty coming from missing higher-order terms from the result at $\mathcal{O}\left(p^{6}\right)$ when only SD constraints are used], we have-for $E_{\gamma}^{\text {cut }}=300 \mathrm{MeV}-\mathrm{BR}\left(\tau^{-} \rightarrow \pi^{-} \pi^{0} \nu_{\tau}\right)=\left(1.9_{-0.0}^{+3.2}\right) \times 10^{-4}$.

The spectrum for these decays with $v_{i}=a_{i}=0$ is plotted in Fig. 10; the dominant peak corresponds to bremsstrahlung off the $\pi^{-}$, and the secondary receives two contributions: one from bremsstrahlung off the $\tau$ lepton and another from a resonance exchange in $V_{\mu \nu}$ (for $E_{\gamma}^{\text {cut }} \leq 100 \mathrm{MeV}$, these two are merged into one single

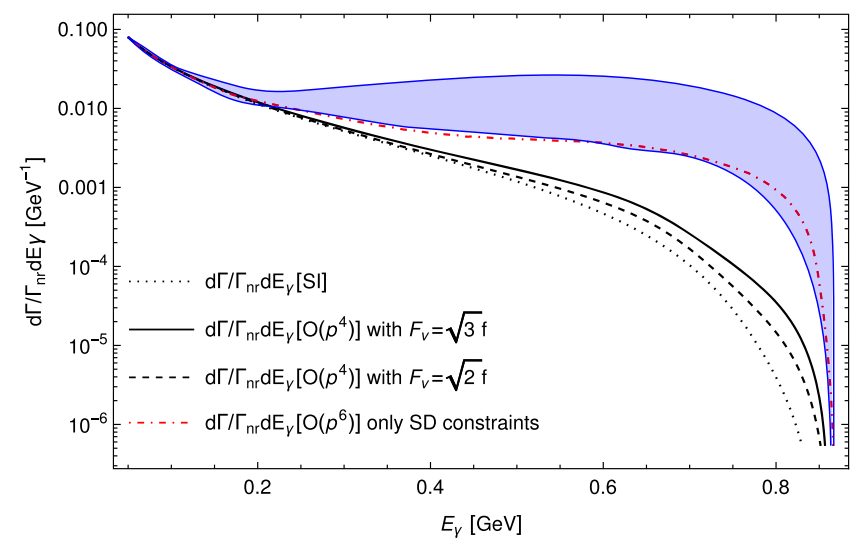

FIG. 13. Photon energy distribution for the $\tau^{-} \rightarrow \pi^{-} \pi^{0} \gamma \nu_{\tau}$ decays normalized with the nonradiative decay width. The dotted line represents the bremsstrahlung contribution. The solid and dashed lines represent the $\mathcal{O}\left(p^{4}\right)$ corrections using $F_{V}=\sqrt{3} F$ and $F_{V}=\sqrt{2} F$, respectively. The dot-dashed red line corresponds to using only SD constraints at $\mathcal{O}\left(p^{6}\right)$ (with overestimated uncertainties in the blue shaded area).

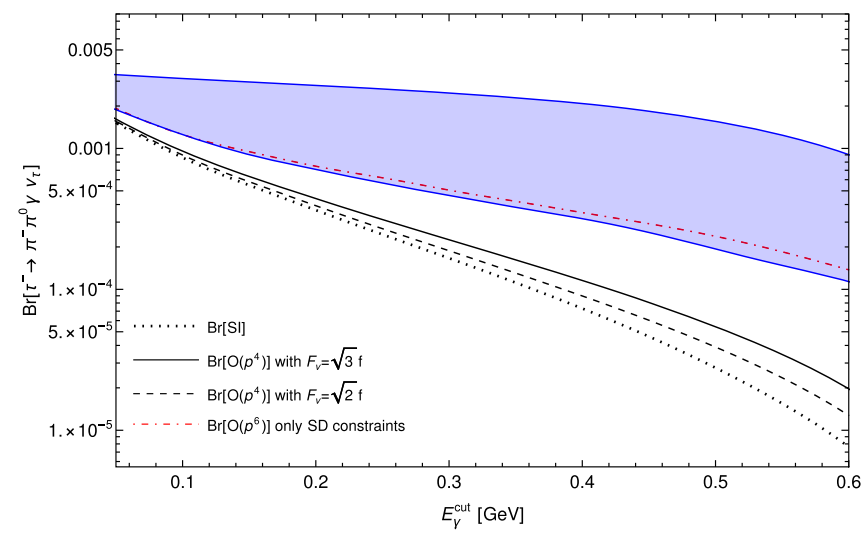

FIG. 14. Branching ratio for the $\tau^{-} \rightarrow \pi^{-} \pi^{0} \gamma \nu_{\tau}$ decays as a function of $E_{\gamma}^{\text {cut }}$. The dotted line represents the bremsstrahlung contribution, and the solid line and dashed lines represent the $\mathcal{O}\left(p^{4}\right)$ corrections using $F_{V}=\sqrt{3} F$ and $F_{V}=\sqrt{2} F$, respectively. The dot-dashed red line is the $\mathcal{O}\left(p^{6}\right)$ contribution using only SD constraints and neglecting all other couplings. The blue shaded region overestimates the $\mathcal{O}\left(p^{6}\right)$ uncertainties.

peak). The rate and spectrum are dominated by the complete bremsstrahlung (SI) contribution.

In Fig. 11, we show the distribution for $E_{\gamma}^{\text {cut }}=300 \mathrm{MeV}$ taking into account the SI contribution (dotted line) and the $\mathcal{O}\left(p^{4}\right)$ amplitude obtained using $F_{V}=\sqrt{2} F$ (dashed line) and $F_{V}=\sqrt{3} F$ (solid line); the most important contribution corresponds to the $\rho$ resonance exchange at $s \sim 0.6 \mathrm{GeV}^{2}$. The main difference between these two approaches is seen in Fig. 11, where up to $s \sim 0.4 \mathrm{GeV}^{2}$ the dashed line is below and the solid line is above the bremsstrahlung (SI) contribution (dotted line). The dashed line is quite similar to the distribution in Fig. 2 of Ref. [62], while the solid line resembles closely the distribution in Fig. 4.6 of Ref. [142] obtained from the VMD model [144] neglecting the $\omega$-resonance contribution.

In Fig. 12, we show a comparison between the dipion distribution at different orders. As we can see, the inclusion of the corrections at $\mathcal{O}\left(p^{6}\right)$ gives a noticeable enhancement at low $s$.

For the photon energy distribution, Fig. 13, we can differentiate between the full amplitude [solid, dashed lines

TABLE II. Branching ratios $\operatorname{Br}\left(\tau^{-} \rightarrow \pi^{-} \pi^{0} \gamma \nu_{\tau}\right)$ for different values of $E_{\gamma}^{\text {cut }}$. The second column corresponds to the complete bremsstrahlung, and the third and fourth columns correspond to the $\mathcal{O}\left(p^{4}\right)$ contributions.

\begin{tabular}{lccc}
\hline \hline & $\begin{array}{c}\mathrm{BR} \\
E_{\gamma}^{\text {cut }}\end{array}$ & $\begin{array}{c}\mathrm{BR}\left(F_{V}=\sqrt{2} F\right) \\
{\left[\mathcal{O}\left(p^{4}\right)\right]}\end{array}$ & $\begin{array}{c}\mathrm{BR}\left(F_{V}=\sqrt{3} F\right) \\
{\left[\mathcal{O}\left(p^{4}\right)\right]}\end{array}$ \\
\hline $100 \mathrm{MeV}$ & $8.6 \times 10^{-4}$ & $9.0 \times 10^{-4}$ & $9.5 \times 10^{-4}$ \\
$300 \mathrm{MeV}$ & $1.7 \times 10^{-4}$ & $1.9 \times 10^{-4}$ & $2.3 \times 10^{-4}$ \\
$500 \mathrm{MeV}$ & $2.8 \times 10^{-5}$ & $3.9 \times 10^{-5}$ & $5.4 \times 10^{-5}$ \\
\hline \hline
\end{tabular}



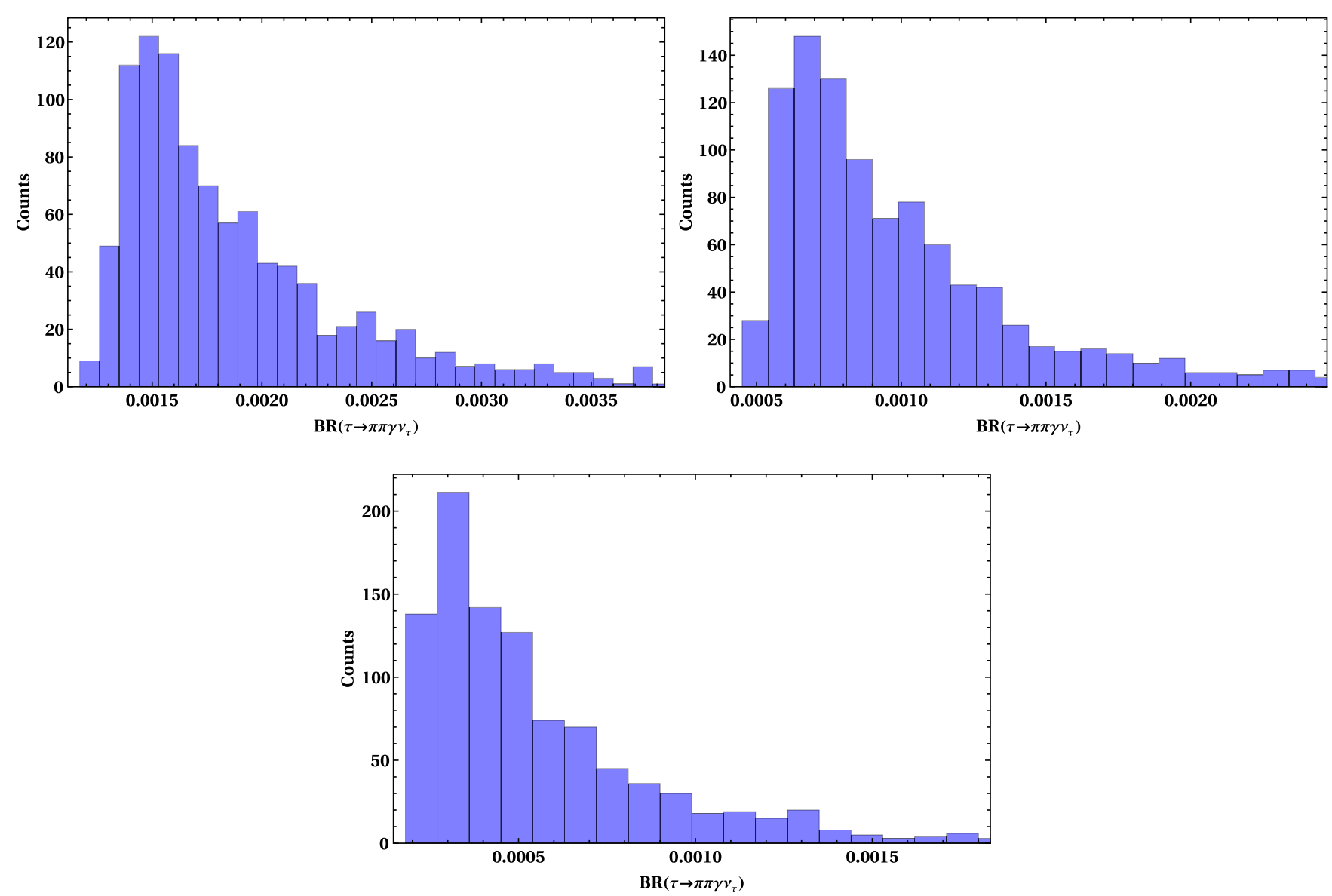

FIG. 15. Predictions for the branching ratio at $\mathcal{O}\left(p^{6}\right)$ for a sample of 1000 points, with $E_{\text {cut }}=100,300$, and $500 \mathrm{MeV}$ from top to bottom.

up to $\mathcal{O}\left(p^{4}\right)$ and dotdashed red line up to $\left.\mathcal{O}\left(p^{6}\right)\right]$ and the bremsstrahlung contribution (dotted line), but as in the case of the branching fraction, the distribution decreases for high energies. In the case of the $\mathcal{O}\left(p^{6}\right)$ distribution, there is an enhancement at middle and high photon energies.

According to Figs. 11-14, measurements of the $\pi \pi$ invariant mass, of the photon spectrum and the partial decay width, for a reasonable cut on $E_{\gamma}$ (at low enough energies the inner bremsstrahlung contribution hides completely any structure-dependent effect), could decrease substantially the uncertainty of the $\mathcal{O}\left(p^{6}\right)$ computation. This was already emphasized in Ref. [62] but remained unmeasured at BABAR and Belle. We hope these data can finally be acquired and analyzed at Belle-II.

TABLE III. Branching ratios $\operatorname{Br}\left(\tau^{-} \rightarrow \pi^{-} \pi^{0} \gamma \nu_{\tau}\right)$ for different $E_{\gamma}^{\text {cut }}$ values at $\mathcal{O}\left(p^{6}\right)$.

\begin{tabular}{lcc}
\hline \hline$E_{\gamma}^{\text {cut }}$ & $\mathrm{BR}(\mathrm{SD})\left[\mathcal{O}\left(p^{6}\right)\right]$ & $\mathrm{BR}\left[\mathcal{O}\left(p^{6}\right)\right]$ \\
\hline $100 \mathrm{MeV}$ & $1.3 \times 10^{-3}$ & $(1.9 \pm 0.3) \times 10^{-3}$ \\
$300 \mathrm{MeV}$ & $5.1 \times 10^{-4}$ & $(1.1 \pm 0.3) \times 10^{-3}$ \\
$500 \mathrm{MeV}$ & $2.4 \times 10^{-4}$ & $(0.6 \pm 0.2) \times 10^{-3}$ \\
\hline \hline
\end{tabular}

In Fig. 15, we show the branching ratio for $E_{\gamma}^{\text {cut }}=$ 100,300 , and $500 \mathrm{MeV}$ from top to bottom. The outcomes are summarized in Table III.

\section{IB CORRECTIONS TO $a_{\mu}^{\mathrm{HVP}, \mathrm{LO} \pi \pi}$}

We can evaluate the leading contributions to the hadronic vacuum polarization (HVP) by means of the dispersion relation [145],

$$
a_{\mu}^{\mathrm{HVP}, \mathrm{LO}}=\frac{1}{4 \pi^{3}} \int_{s_{t h r}}^{\infty} d s K(s) \sigma_{e^{-} e^{+} \rightarrow \text { hadrons }}^{0}(s),
$$

where $K(s)$ is a smooth QED kernel concentrated at low energies, which increases the $E \lesssim M_{\rho}$ contribution,

$$
\begin{aligned}
& \begin{aligned}
K(s)= & \frac{x^{2}}{2}\left(2-x^{2}\right)+\frac{\left(1+x^{2}\right)(1+x)^{2}}{x^{2}}\left(\ln (1+x)-x+\frac{x^{2}}{2}\right) \\
& +\frac{(1+x)}{(1-x)} x^{2} \ln (x),
\end{aligned} \\
& \text { with }
\end{aligned}
$$


TABLE IV. Contributions to $\Delta a_{\mu}^{\mathrm{HVP}, \mathrm{LO}}$ in units of $10^{-11}$ using the dispersive representation of the form factor. From the two evaluations labeled $\mathcal{O}\left(p^{4}\right)$, the left (right) one corresponds to $F_{V}=\sqrt{2} F\left(F_{V}=\sqrt{3} F\right)$.

\begin{tabular}{lcccccc}
\hline \hline$\left[s_{1}, s_{2}\right]$ & $\Delta a_{\mu, \mathrm{G}_{\mathrm{EM}}^{(0)}}^{\mathrm{HVO}}$ & $\Delta a_{\mu, \mathrm{SI}}^{\mathrm{HVP}, \mathrm{LO}}$ & $\Delta a_{\mu,\left[\mathcal{O}\left(p^{4}\right)\right]}^{\mathrm{HVPO}}$ & $\Delta a_{\mu,\left[\mathcal{O}\left(p^{4}\right)\right]}^{\mathrm{HVO}, \mathrm{LO}}$ & $\Delta a_{\mu,[S D]}^{\mathrm{HUP}, \mathrm{LO}}$ & $\Delta a_{\mu,\left[\mathcal{O}\left(p^{6}\right)\right]}^{\mathrm{HVP}, \mathrm{LO}}$ \\
\hline$\left[4 m_{\pi}^{2}, 1 \mathrm{GeV}^{2}\right]$ & +17.8 & -11.0 & -11.3 & -17.0 & -32.4 & $-74.8 \pm 44.0$ \\
{$\left[4 m_{\pi}^{2}, 2 \mathrm{GeV}^{2}\right]$} & +18.3 & -10.1 & -10.3 & -16.0 & -31.9 & $-75.9 \pm 45.5$ \\
{$\left[4 m_{\pi}^{2}, 3 \mathrm{GeV}^{2}\right]$} & +18.4 & -10.0 & -10.2 & -15.9 & -31.9 & $-75.9 \pm 45.6$ \\
{$\left[4 m_{\pi}^{2}, m_{\tau}^{2}\right]$} & +18.4 & -10.0 & -10.2 & -15.9 & -31.9 & $-75.9 \pm 45.6$ \\
\hline \hline
\end{tabular}

TABLE V. Contributions to $\Delta a_{\mu}^{\mathrm{HVP}, \mathrm{LO}}$ in units of $10^{-11}$ using the Guerrero-Pich parametrization of the form factor. From the two evaluations labeled $\mathcal{O}\left(p^{4}\right)$, the left (right) one corresponds to $F_{V}=\sqrt{2} F\left(F_{V}=\sqrt{3} F\right)$.

\begin{tabular}{|c|c|c|c|c|c|c|}
\hline$\left[s_{1}, s_{2}\right]$ & $\Delta a_{\mu, \mathrm{G}_{\mathrm{EM}}^{(0)}}^{\mathrm{HVP}, \mathrm{LO}}$ & $\Delta a_{\mu, \mathrm{SI}}^{\mathrm{HVP}, \mathrm{LO}}$ & $\Delta a_{\mu,\left[\mathcal{O}\left(p^{4}\right)\right]}^{\mathrm{HVP}, \mathrm{LO}}$ & $\Delta a_{\mu,\left[\mathcal{O}\left(p^{4}\right)\right]}^{\mathrm{HVP}, \mathrm{LO}}$ & $\Delta a_{\mu,[S D]}^{\mathrm{HVP}, \mathrm{LO}}$ & $\Delta a_{\mu,\left[\mathcal{O}\left(p^{6}\right)\right]}^{\mathrm{HVP}, \mathrm{LO}}$ \\
\hline$\left[4 m_{\pi}^{2}, 1 \mathrm{GeV}^{2}\right]$ & +17.3 & -10.2 & -10.4 & -15.9 & -28.3 & $-63.2 \pm 16.5$ \\
\hline$\left[4 m_{\pi}^{2}, 2 \mathrm{GeV}^{2}\right]$ & +17.7 & -9.4 & -9.6 & -15.2 & -28.1 & $-58.1 \pm 12.2$ \\
\hline$\left[4 m_{\pi}^{2}, 3 \mathrm{GeV}^{2}\right]$ & +17.8 & -9.3 & -9.5 & -15.1 & -28.0 & $-67.8 \pm 17.5$ \\
\hline$\left[4 m_{\pi}^{2}, m_{\tau}^{2}\right]$ & +17.8 & -9.3 & -9.5 & -15.1 & -28.0 & $-64.9 \pm 13.4$ \\
\hline
\end{tabular}

$$
x=\frac{1-\beta_{\mu}}{1+\beta_{\mu}}, \quad \beta_{\mu}=\sqrt{1-4 m_{\mu}^{2} / s},
$$

and $\sigma_{e^{-} e^{+} \rightarrow \text { hadrons }}^{0}(s)$ is the bare hadronic cross section. ${ }^{28} \mathrm{We}$ can relate the hadronic spectral function from $\tau$ decays to the $e^{+} e^{-}$hadronic cross section by including the radiative corrections and the IB effects. For the $\pi \pi$ final state, we have $[61,62]$

$$
\sigma_{\pi \pi}^{0}=\left[\frac{K_{\sigma}(s)}{K_{\Gamma}(s)} \frac{d \Gamma_{\pi \pi[\gamma]}}{d s}\right] \frac{R_{\mathrm{IB}}(s)}{S_{\mathrm{EW}}},
$$

where

$$
\begin{aligned}
& K_{\Gamma}(s)=\frac{G_{F}^{2}\left|V_{u d}\right|^{2} m_{\tau}^{3}}{384 \pi^{3}}\left(1-\frac{s}{m_{\tau}^{2}}\right)^{2}\left(1+\frac{2 s}{m_{\tau}^{2}}\right), \\
& K_{\sigma}(s)=\frac{\pi \alpha^{2}}{3 s}
\end{aligned}
$$

and the IB corrections

$$
R_{\mathrm{IB}}(s)=\frac{F S R(s)}{G_{\mathrm{EM}}(s)} \frac{\beta_{\pi^{+} \pi^{-}}^{3}}{\beta_{\pi^{+} \pi^{0}}^{3}}\left|\frac{F_{V}(s)}{f_{+}(s)}\right|^{2} .
$$

The $S_{\mathrm{EW}}$ term encodes the SD electroweak corrections [146-153], and $F S R(s)$ accounts for the radiation from the final-state pions $[154,155]$. The $G_{\mathrm{EM}}(s)$ term was already

\footnotetext{
${ }^{28}$ Although final-state radiation would belong to HVP, NLO, it is always included in HVP, LO (and not in HVP, NLO) as eliminating this radiation from the measured data is unfeasible. Thus, a final-state radiation factor is also needed in the radiative corrections discussed below.
}

discussed at length in Sec. III, the $\beta_{\pi^{+} \pi^{-}}^{3} / \beta_{\pi^{+} \pi^{0}}^{3}$ term is a phase space factor, and the last term in $R_{\mathrm{IB}}(s)$ is a ratio between the neutral $\left[F_{V}(s)\right]$ and the charged $\left[f_{+}(s)\right]$ pion form factor.

To study the effect of the radiative correction $G_{\mathrm{EM}}(s)$ on $a_{\mu}^{\mathrm{HVP}, \mathrm{LO}}[\pi \pi]$, we have evaluated the expression [62]

$$
\Delta a_{\mu}^{\mathrm{HVP}, \mathrm{LO}}=\frac{1}{4 \pi^{3}} \int_{s_{1}}^{s_{2}} d s K(s)\left[\frac{K_{\sigma}(s)}{K_{\Gamma}(s)} \frac{d \Gamma_{\pi \pi[\gamma]}}{d s}\right]\left(\frac{R_{\mathrm{IB}}(s)}{S_{\mathrm{EW}}}-1\right),
$$

taking $S_{\mathrm{EW}}=1, \frac{\beta_{\pi^{+} \pi^{-}}^{3}}{\beta_{\pi^{+} \pi^{0}}^{3}}=1$ and $\left|\frac{F_{V}(s)}{f_{+}(s)}\right|^{2}=1$. The results are summarized in Table IV using dispersion relation (DR) form factor. The results obtained for the $G_{\mathrm{EM}}^{(0)}$ and the complete $\mathcal{O}\left(p^{4}\right)$ contribution (with $F_{V}=\sqrt{2} F$ ) agree with those in Ref. [62], which are $+16 \times 10^{-11}$ and $-10 \times 10^{-11}$, respectively (for the whole integral). In Table V, we summarize the results obtained using the Guerrero-Pich [143] parametrization of the form factor (which only accounts for the completely dominant $\rho$ exchange), which are in nice agreement with those found with the dispersive form factor [that also includes the $\rho(1450)$ and $\rho(1700)$ effects]. This checks, a posteriori, that excited resonance contributions make a negligible effect in the $G_{\mathrm{EM}}(s)$ corrections to $a_{\mu}^{\mathrm{HVP}, \mathrm{LO}} .29$

\footnotetext{
${ }^{29}$ By replacing $D_{\rho}^{-1}(x)$ by $\left(1+\beta_{\rho^{\prime}}\right)^{-1}\left(D_{\rho}^{-1}(x)+\beta_{\rho^{\prime}} D_{\rho^{\prime}}^{-1}(x)\right)$, with $\beta_{\rho^{\prime}} \in[0.12,0.15]$ [125] throughout the $v_{i}$ and $a_{i}$ form factors, we have verified that the impact of the $\rho^{\prime}$ on the $G_{\mathrm{EM}}(s)$ correction to $a_{\mu}^{\mathrm{HVP},\left.\mathrm{LO}\right|_{\pi \pi, \tau}}$ is negligible. Similarly, the error induced by other excited resonances shall also be irrelevant.
} 


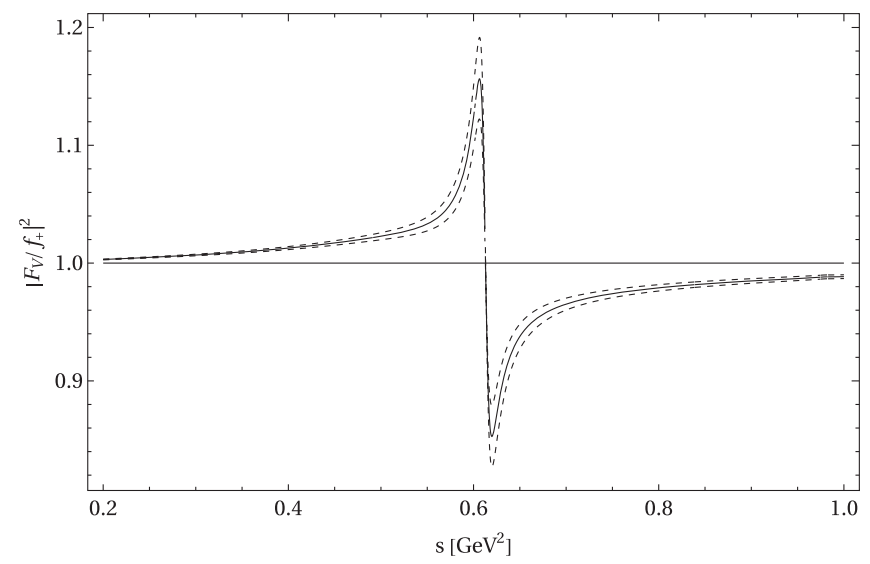

FIG. 16. Ratio of the form factors (FF1) for $\theta_{\rho \omega}=$ $(-3.5 \pm 0.7) \times 10^{-3} \mathrm{GeV}^{2}$. The solid line represents the mean value.

The values in the last columns of Tables IV and V were obtained evaluating Eq. (70) according to the couplings discussed in Sec. IIE for a sample of 200 points for each interval of integration (results were stable under increasing this number).

The other contributions are summarized in Table VI:

(i) The $S_{\mathrm{EW}}$ contribution $S_{\mathrm{EW}}=1.0201$ gives $\Delta a_{\mu}^{\mathrm{HVP}, \mathrm{LO}}=-103.1 \times 10^{-11}$, consistent with earlier determinations (using slightly different values of $\left.S_{\mathrm{EW}}\right)$ and with a negligible error.

(ii) The phase space (PS) correction induces $\Delta a_{\mu}^{\mathrm{HVP}, \mathrm{LO}}=$ $-74.5 \times 10^{-11}$ (trivially in agreement with previous computations), again with tiny uncertainties.

(iii) The final-state radiation (FSR, which is formally NLO) yields $\Delta a_{\mu}^{\mathrm{HVP}, \mathrm{LO}}=+45.5(4.6) \times 10^{-11}$, in accord with Ref. [67] (its value was not quoted in Ref. [62]).

(iv) The correction due to the ratio of the form factors (Fig. 16) is harder to evaluate. We have considered two alternatives, labeled FF1 and FF2, that we explain next. We use the following numerical inputs for the $\rho-\omega$ mixing parameter $\theta_{\rho \omega}=$ $(-3.5 \pm 0.7) \times 10^{-3} \mathrm{GeV}^{2} \quad$ [62] and $\Gamma_{\rho^{0}}-\Gamma_{\rho^{+}}=$ $0.3 \pm 1.3 \mathrm{MeV}, m_{\rho^{ \pm}}-m_{\rho^{0}}=0.7 \pm 0.8 \mathrm{MeV}$, and $m_{\rho^{0}}=775.26 \pm 0.25 \mathrm{MeV}$ from particle data group [156].

In FF1, as in Ref. [62], we include the measurement of the $\pi \pi \gamma$ channel of the $\rho^{0} \Gamma_{\rho^{0} \rightarrow \pi^{+} \pi^{-} \gamma}=$ $1.5 \pm 0.2 \mathrm{MeV}$ and the measurements of $\Gamma_{\rho^{0} \rightarrow \pi^{0} \gamma}$ and $\Gamma_{\rho^{+} \rightarrow \pi^{+} \gamma}$, which are approximately $0.1 \mathrm{MeV}$ [157]. Thus, we estimate $\Gamma_{\rho^{0} \rightarrow \pi^{+} \pi^{-} \gamma}-\Gamma_{\rho^{ \pm} \rightarrow \pi^{ \pm} \pi^{0} \gamma}=$ $1.5 \pm 1.3 \mathrm{MeV}$. In this way, we get a positive correction of $\Delta a_{\mu}^{\mathrm{HVP}, \mathrm{LO}}=+40.9(48.9) \times 10^{-11}$. The uncertainty on the third column of Table VI (FF1) corresponds to sum the errors due to uncertainties of $\rho-\omega$ mixing (8.5), the $\rho^{+}-\rho^{0}$ mass difference (15.9), and the $\rho^{+}-\rho^{0}$ width difference (45.5) in quadrature (in units of $10^{-11}$ ).

On the other hand, in FF2, we use the same numerical inputs for $\Gamma_{\rho^{0} \rightarrow \pi^{+} \pi^{-} \gamma}-\Gamma_{\rho^{ \pm} \rightarrow \pi^{ \pm} \pi^{0} \gamma}=$ $0.45 \pm 0.45 \mathrm{MeV}$ as in Ref. [62] (and all the others as we did before), and we obtain a positive correction of $\Delta a_{\mu}^{\mathrm{HVP}, \mathrm{LO}}=+77.6(24.0) \times 10^{-11}$. The uncertainty in the fourth column of Table VI (FF2) corresponds to sum the errors due to uncertainties of $\rho-\omega$ mixing (8.6), the $\rho^{+}-\rho^{0}$ mass difference (15.9), and the $\rho^{+}-\rho^{0}$ width difference (15.8) in quadrature (in units of $10^{-11}$ ).

This correction is $+(61 \pm 26 \pm 3) \times 10^{-11}$ in Ref. [62] and $+(86 \pm 32 \pm 7) \times 10^{-11}$ in Ref. [67], in agreement (despite the big errors) with our FF2 and FF1 determinations, respectively.

(v) Finally, we get $\left(-15.9_{-16.0}^{+5.7}\right) \times 10^{-11}[(-76 \pm 46) \times$ $10^{-11}$ ] for the $G_{\mathrm{EM}}(s)$ correction at $\mathcal{O}\left(p^{4}\right)\left[\mathcal{O}\left(p^{6}\right)\right]$ versus $-10 \times 10^{-11}$ in Ref. [62] and $-37 \times 10^{-11}$ in Ref. [65] (from the last two results, $(-19.2 \pm 9.0) \times$ $10^{-11}$ was used in Ref. [67]). As explained before, the previous uncertainty on the $\mathcal{O}\left(p^{6}\right)$ can only be taken as an upper bound on it. Also interesting is the $G_{\mathrm{EM}}(s)$ correction when only the couplings restricted by SD are used (with all others at this order set to zero), which allows us to estimate the effect of missing higher-order terms on the $\mathcal{O}\left(p^{4}\right)$ result quoted above. This $\mathcal{O}\left(p^{4}\right)$ result, which is our reference value, is consistent with both the earlier $R \chi T$ [62] and the VMD [67] evaluations, albeit with a larger (asymmetric) error.

In Fig. 17, we show the full IB correction factor $R_{\mathrm{IB}}(s)$ for the different orders of approximation in the $G_{\mathrm{EM}}(s)$ factor using the DR parametrization of the form factor. As we can see, there is a difference between the contributions at $\mathcal{O}\left(p^{4}\right)$ and those at $\mathcal{O}\left(p^{6}\right)$ for energies below approximately $0.5 \mathrm{GeV}^{2}$ and above approximately $0.7 \mathrm{GeV}^{2}$.

An important cross-check is the branching fraction $B_{\pi \pi^{0}}=\Gamma\left(\tau \rightarrow \pi \pi^{0} \nu_{\tau}\right) / \Gamma_{\tau}$, which is a directly measured quantity. It can also be evaluated from the $I=1$ component of the $e^{+} e^{-} \rightarrow \pi^{+} \pi^{-}(\gamma)$ cross section after taking into account the IB corrections. The branching fraction is given by

$$
B_{\pi \pi^{0}}^{\mathrm{CVC}}=B_{e} \int_{4 m_{\pi}^{2}}^{m_{\tau}^{2}} d s \sigma_{\pi^{+} \pi^{-}(\gamma)}(s) \mathcal{N}(s) \frac{S_{\mathrm{EW}}}{R_{\mathrm{IB}}(s)},
$$

where

$$
\mathcal{N}(s)=\frac{3\left|V_{u d}\right|^{2}}{2 \pi \alpha_{0}^{2} m_{\tau}^{2}} s\left(1-\frac{s}{m_{\tau}^{2}}\right)^{2}\left(1+\frac{2 s}{m_{\tau}^{2}}\right) .
$$


TABLE VI. Contributions to $\Delta a_{\mu}^{\mathrm{HVP}, \mathrm{LO}}$ in units of $10^{-11}$ using the DR form factor as the reference one.

\begin{tabular}{|c|c|c|c|c|c|c|}
\hline$\left[s_{1}, s_{2}\right]$ & $\mathrm{S}_{\mathrm{EW}}$ & PS & FSR & FF1 & FF2 & EM \\
\hline$\left[4 m_{\pi}^{2}, 1 \mathrm{GeV}^{2}\right]$ & -101.1 & -74.1 & +44.7 & $+41.8 \pm 49.0$ & $+78.4 \pm 24.5$ & $-17.0_{-15.4}^{+5.7}$ \\
\hline$\left[4 m_{\pi}^{2}, 2 \mathrm{GeV}^{2}\right]$ & -103.1 & -74.4 & +45.5 & $+40.9 \pm 48.9$ & $+77.6 \pm 24.0$ & $\begin{array}{l}-16.0_{-15.9}^{+5.4} \\
-5\end{array}$ \\
\hline$\left[4 m_{\pi}^{2}, 3 \mathrm{GeV}^{2}\right]$ & -103.1 & -74.5 & +45.5 & $+40.9 \pm 48.9$ & $+77.6 \pm 24.0$ & $-15.9_{-16.0}^{+5.9}$ \\
\hline$\left[4 m_{\pi}^{2}, m_{\tau}^{2}\right]$ & -103.1 & -74.5 & +45.5 & $+40.9 \pm 48.9$ & $+77.6 \pm 24.0$ & $-15.9_{-16.0}^{+5.7}$ \\
\hline$\left[s_{1}, s_{2}\right]$ & \multicolumn{5}{|c|}{$\Delta a_{\mu}(\mathrm{FF} 1)$} & $\Delta a_{\mu}(\mathrm{FF} 2)$ \\
\hline$\left[4 m_{\pi}^{2}, 1 \mathrm{GeV}^{2}\right]$ & \multicolumn{5}{|c|}{$-105.7_{-51.6}^{+49.5}$} & $-69.1_{-29.3}^{+25.6}$ \\
\hline$\left[4 m_{\pi}^{2}, 2 \mathrm{GeV}^{2}\right]$ & \multicolumn{5}{|c|}{$-107.1_{-51.6}^{+49.0}$} & $-70.4_{-29.2}^{+25.1}$ \\
\hline$\left[4 m_{\pi}^{2}, 3 \mathrm{GeV}^{2}\right]$ & \multicolumn{5}{|c|}{$-107.1_{-51.7}^{+49.4}$} & $-70.4_{-29.2}^{+25.1}$ \\
\hline$\left[4 m_{\pi}^{2}, m_{\tau}^{2}\right]$ & \multicolumn{5}{|c|}{$-107.1_{-51.7}^{+49.4}$} & $-70.4_{-29.2}^{+25.1}$ \\
\hline
\end{tabular}

Using the most recent data obtained from $B A B A R[54]^{30}$ for the $e^{+} e^{-} \rightarrow \pi^{+} \pi^{-}(\gamma)$ cross section and taking the same numerical inputs as we did for FF1, we get

$$
B_{\pi \pi^{0}}^{\mathrm{CVC}}= \begin{cases}(24.76 \pm 0.11 \pm 0.25 \pm 0.01 \pm 0.01 \pm 0.02) \%, & \text { SI } \\ (24.77 \pm 0.11 \pm 0.25 \pm 0.01 \pm 0.01 \pm 0.02) \%, & F_{V}=\sqrt{2} F \\ (24.77 \pm 0.11 \pm 0.25 \pm 0.01 \pm 0.01 \pm 0.02) \%, & F_{V}=\sqrt{3} F \\ (24.80 \pm 0.11 \pm 0.25 \pm 0.01 \pm 0.01 \pm 0.02) \%, & \text { SD }\end{cases}
$$

where SI, $F_{V}=\sqrt{2} F, F_{V}=\sqrt{2} F$, and SD correspond to the different approximations of the $G_{\mathrm{EM}}(s)$ factor. The result for $F_{V}=\sqrt{2} F$ is our reference one, with a negligible uncertainty from the missing higher-order terms starting at $\mathcal{O}\left(p^{6}\right)$.

On the other hand, when we use the same numerical inputs as in the case of FF2, we get (again, our reference result is the $F_{V}=\sqrt{2} F$ one, with the uncertainties quoted below)

$$
B_{\pi \pi^{0}}^{\mathrm{CVC}}= \begin{cases}(24.57 \pm 0.11 \pm 0.08 \pm 0.01 \pm 0.01 \pm 0.02) \%, & \text { SI } \\ (24.57 \pm 0.11 \pm 0.08 \pm 0.01 \pm 0.01 \pm 0.02) \%, & F_{V}=\sqrt{2} F \\ (24.58 \pm 0.11 \pm 0.08 \pm 0.01 \pm 0.01 \pm 0.02) \%, & F_{V}=\sqrt{3} F \\ (24.61 \pm 0.11 \pm 0.08 \pm 0.01 \pm 0.01 \pm 0.02) \%, & \text { SD }\end{cases}
$$

In both cases, the first error corresponds to the statistical experimental uncertainty on $\sigma_{\pi \pi(\gamma)}$, the second is related to uncertainty on the $\rho^{+}-\rho^{0}$ width difference, the third corresponds to the uncertainty in the $\rho^{+}-\rho^{0}$ mass difference, and the fourth corresponds to the uncertainty of the $\rho-\omega$ mixing. The last error corresponds to the corrections induced by FSR on $B_{\pi \pi^{0}}^{\mathrm{CVC}}$, which reduces approximately $-0.20(2) \%$ the $\pi \pi$ branching fraction.

If we include all the couplings contributing to $G_{\mathrm{EM}}(s)$ at $\mathcal{O}\left(p^{6}\right)$ according to Sec. II E, we have an additional error associated to the electromagnetic (EM) contributions. Thus, we get

$$
B_{\pi \pi^{0}}^{\mathrm{CVC}}=\left(24.80 \pm 0.11 \pm 0.25 \pm 0.01 \pm 0.01 \pm 0.02_{-0.01}^{+0.21}\right) \%
$$

for FF1 and

$$
B_{\pi \pi^{0}}^{\mathrm{CVC}}=\left(24.61 \pm 0.11 \pm 0.08 \pm 0.01 \pm 0.01 \pm 0.02_{-0.01}^{+0.21}\right) \%
$$

for FF2. Both previous results match perfectly our reference determinations obtained with $F_{V}=\sqrt{2} F$.

\footnotetext{
${ }^{30}$ We thank to Alex Keshavarzi and Bogdan Malaescu for providing us tables with the measurement of the $e^{+} e^{-} \rightarrow \pi^{+} \pi^{-}(\gamma)$ cross section.
} 

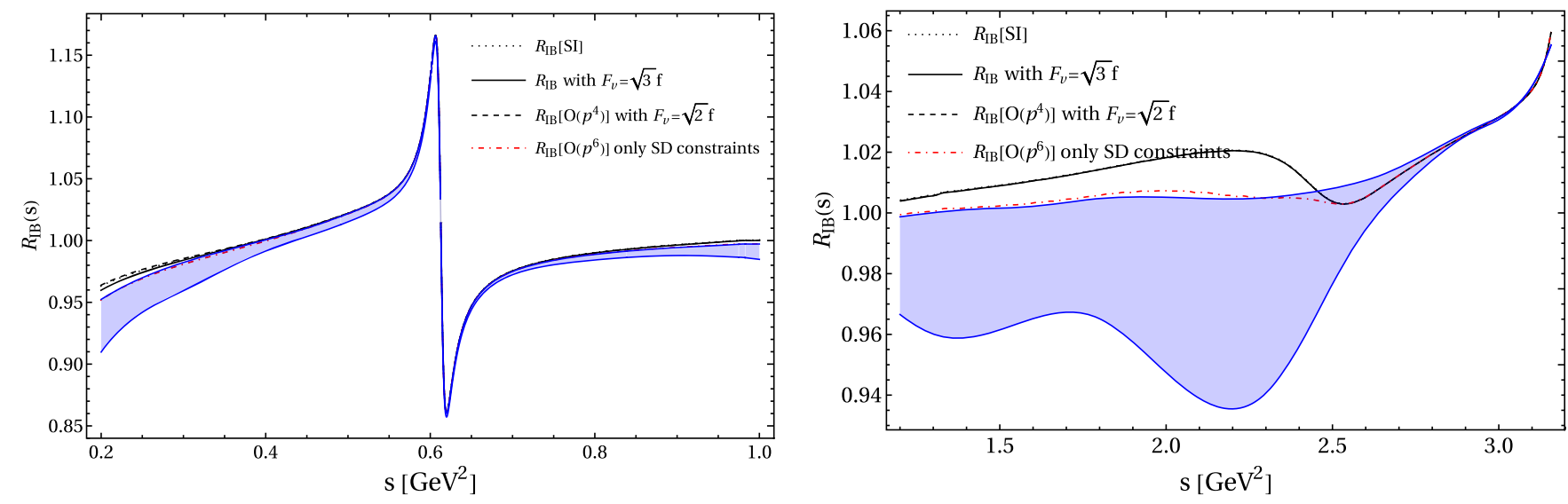

FIG. 17. Full IB correction factor $R_{\mathrm{IB}}(s)$ for the different orders of approximation in $G_{\mathrm{EM}}(s)$ using the central values given in (FF1). The blue region corresponds to the (overestimated) corrections at $\mathcal{O}\left(p^{6}\right)$ in $G_{\mathrm{EM}}(s)$.

These results are in good agreement (though better for FF1) with the value reported by the Belle Collaboration [158],

$$
B_{\pi \pi^{0}}^{\tau}=(25.24 \pm 0.01 \pm 0.39) \%,
$$

where the first uncertainty is statistical and the second is systematic. Nonetheless, they are in some tension with the very precise ALEPH measurement $(25.471 \pm 0.097 \pm$ $0.085) \%$ [159].

We show in Fig. 18 the prediction for the $e^{+} e^{-} \rightarrow \pi^{+} \pi^{-}$ cross section using the data reported by Belle [158] (as it is the most precise measurement of this spectrum) for the normalized spectrum $\left(1 / N_{\pi \pi}\right)\left(d N_{\pi \pi} / d s\right)$ compared to the last measurements from BABAR [54] and KLOE [160]. ${ }^{31}$

We recall that the $e^{+} e^{-} \rightarrow \pi^{+} \pi^{-}$cross section obtained using $\tau$ data is given by [158]

$\sigma_{\pi \pi}^{0}=\frac{1}{\mathcal{N}(s)} \times\left(\frac{B_{\pi \pi}}{B_{e}}\right) \times\left(\frac{1}{N_{\pi \pi}} \frac{d N_{\pi \pi}}{d s}\right)\left(\frac{R_{\mathrm{IB}}(s)}{S_{\mathrm{EW}}}\right)$.

In Fig. 18, the $\tau$-based prediction is obtained using the $\mathcal{O}\left(p^{4}\right)$ result for $G_{\mathrm{EM}}(s)$, with the estimated uncertainty from missing higher-order corrections given by the result at $\mathcal{O}\left(p^{6}\right)$ (employing only the SD constraints). The blue dotdashed line shown overestimates the error at $\mathcal{O}\left(p^{6}\right)$.

From Fig. 18, we observe good agreement between the $B A B A R$ data and the $\tau$ decays prediction (slightly better for FF1). ${ }^{32}$ The previous comparisons make us consider our evaluation with FF1 the reference one (so that its difference

\footnotetext{
${ }^{31} \mathrm{We}$ have chosen to show in the comparison these two $e^{+} e^{-}$ datasets as the results from both collaborations are those deviating the most, and thus mainly responsible from the tension in $\sigma\left(e^{+} e^{-} \rightarrow \pi^{+} \pi^{-}\right)$.

${ }^{32}$ One can also check how important the $\rho^{+}-\rho^{0}$ width difference is around $s \simeq M_{\rho}^{2}$.
}

with FF2 will assess the size of the error induced by IB among the $\rho \rightarrow \pi \pi \gamma$ decay channels). ${ }^{33}$

Using Eq. (78), we evaluate the IB-corrected $a_{\mu}^{\mathrm{HVP}, \mathrm{LO}}[\pi \pi, \tau]$ from the Belle mass spectrum. We use the particle data group values [156] for $m_{\tau}, V_{u d}$, and $B_{e}$.

In Table VII (VIII) we show IB-corrected $a_{\mu}^{\mathrm{HVP}, \mathrm{LO}}[\pi \pi, \tau]$ in units of $10^{-10}$ using the measured mass spectrum by Belle (ALEPH). For each dataset, results for the different approximations to $G_{\mathrm{EM}}(s)$ are shown. We choose showing first the results with both Belle and ALEPH datasets as the first (second) one yields the most accurate spectral function (branching ratio) measurement. As in Ref. [67] (and later works by the Orsay group), the contributions are split in two intervals. In the first one, $\sqrt{s} \in\left[2 m_{\pi^{ \pm}}, 0.36 \mathrm{GeV}\right]$, (the very scarce) data are not used, as they affect the precision of the integral. Instead, we use the results of the dispersive fits in Ref. [125]. We proceed analogously in Tables IX and X with the CLEO [166] and OPAL [167] $]^{34}$ measurements.

Taking into account all dipion tau decay data from the ALEPH [159], Belle [158], CLEO [166], and OPAL [167] collaborations (the latter yielding the largest contribution to $\left.a_{\mu}^{\mathrm{HVP}, \mathrm{LO}}\right|_{\pi \pi}$ exceeding approximately $10.7 \times 10^{-10}$ the mean, although with the largest errors as well) in Tables XI and XII at $\mathcal{O}\left(p^{4}\right)$ and $\mathcal{O}\left(p^{6}\right)$, respectively, we get the combined tau-data contribution

$$
\left.10^{10} \cdot a_{\mu}^{\mathrm{HVP}, \mathrm{LO}}\right|_{\pi \pi, \tau \mathrm{data}}=519.6 \pm 2.8_{\text {spectra }+\mathrm{BRs}_{-2.1 \mathrm{IB}}}^{+1.9}
$$

at $\mathcal{O}\left(p^{4}\right)$ and

\footnotetext{
${ }^{33} \mathrm{We}$, nevertheless, recall that recent BESIII data $[55,161]$ and evaluations within the hidden local symmetry model [162-165] agree better with the KLOE data than with $B A B A R$ 's.

${ }^{34}$ We thank to Jorge Portolés for providing us with the OPAL dataset.
} 

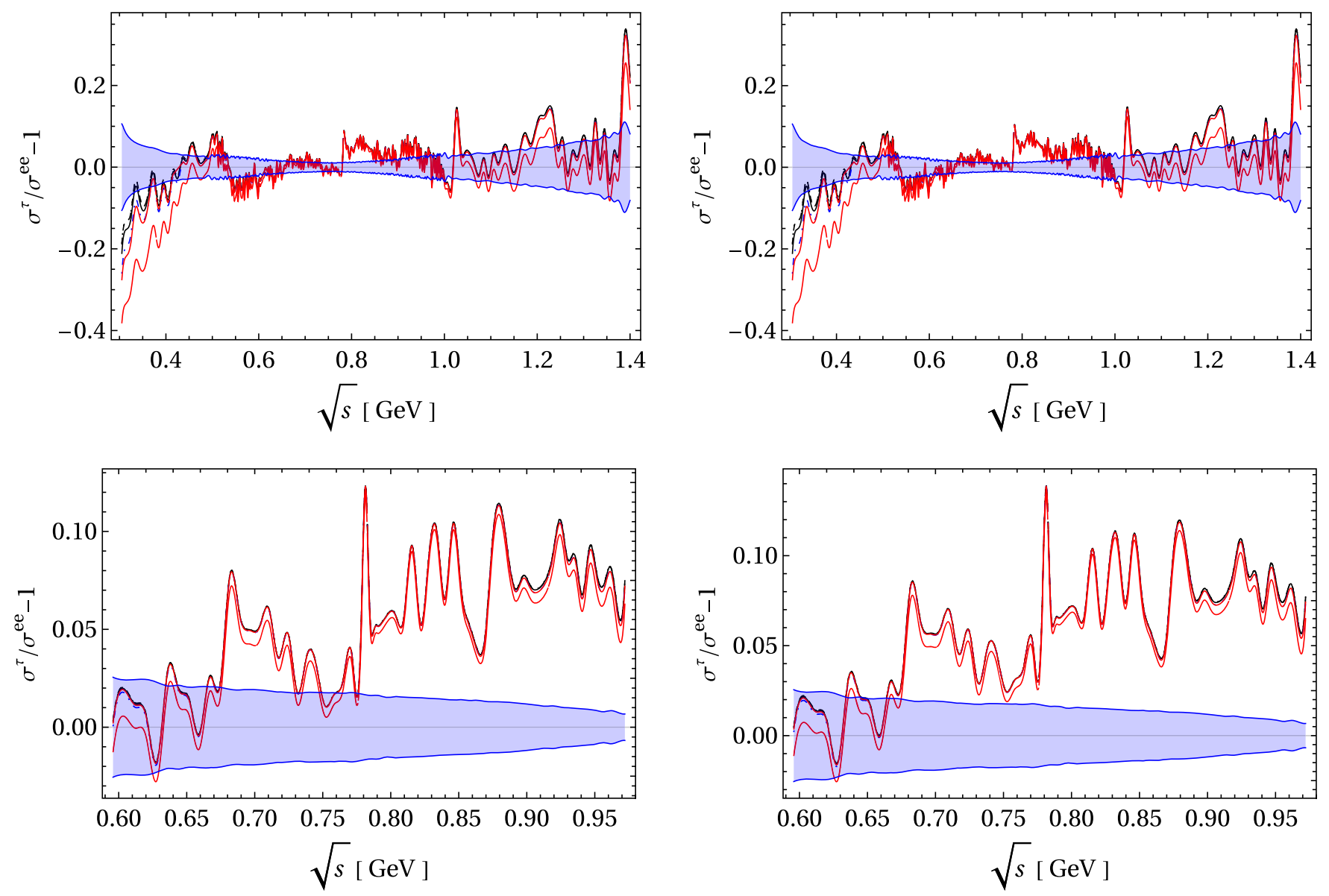

FIG. 18. Comparison between the different data sets from $B A B A R$ (above) and KLOE (below) with $\Delta \Gamma_{\pi \pi \gamma}=1.5 \mathrm{MeV}$ (left) and $\Delta \Gamma_{\pi \pi \gamma}=0.45 \mathrm{MeV}$ (right) for FF1 and FF2, respectively. The blue region corresponds to the experimental error on $\sigma_{\pi \pi(\gamma)}$. The solid and dashed lines represent the contributions with $F_{V}=\sqrt{3} F$ and $F_{V}=\sqrt{2} F$ at $\mathcal{O}\left(p^{4}\right)$, respectively. The dotted line is the SI contribution. The red line depicts the envelope of $G_{\mathrm{EM}}(s)$ at $\mathcal{O}\left(p^{6}\right)$, which overestimates the uncertainty at this order. The blue dot-dashed line is the $\mathcal{O}\left(p^{6}\right)$ contribution using only SD constraints.

TABLE VII. IB-corrected $a_{\mu}^{\mathrm{HVP}, \mathrm{LO}}[\pi \pi, \tau]$ in units of $10^{-10}$ using the measured mass spectrum by Belle with $B_{\pi \pi}=(25.24 \pm 0.01 \pm 0.39) \%$. Different approximations to $G_{\mathrm{EM}}(s)$ are displayed in the various columns. The last three of them show the results at $\mathcal{O}\left(p^{6}\right)$, and their differences overestimate the error at this order. The error of the $\mathcal{O}\left(p^{4}\right)$ prediction (obtained with $\left.F_{V}=\sqrt{2} F\right)$ can be quantified from its difference with the SD value [corresponding to the $\mathcal{O}\left(p^{6}\right)$ contribution using only $\mathrm{SD}$ constraints].

\begin{tabular}{|c|c|c|c|c|c|c|c|}
\hline \multicolumn{8}{|c|}{ FF1 } \\
\hline$\left[s_{1}, s_{2}\right]$ & SI & $F_{V}=\sqrt{2} F$ & $F_{V}=\sqrt{3} F$ & SD & Min & Max & Mean \\
\hline$\left[0.1296 \mathrm{GeV}^{2}, 1 \mathrm{GeV}^{2}\right]$ & 499.43 & 499.42 & 499.05 & 498.16 & 492.18 & 498.41 & $\overline{495.30}$ \\
\hline$\left[0.1296 \mathrm{GeV}^{2}, 2 \mathrm{GeV}^{2}\right]$ & 509.47 & 509.46 & 509.09 & 508.14 & 501.87 & 508.40 & 505.13 \\
\hline$\left[0.1296 \mathrm{GeV}^{2}, 3 \mathrm{GeV}^{2}\right]$ & 509.68 & 509.67 & 509.30 & 508.35 & 502.08 & 508.61 & 505.34 \\
\hline$\left[0.1296 \mathrm{GeV}^{2}, 3.125 \mathrm{GeV}^{2}\right]$ & 509.72 & 509.71 & 509.34 & 508.40 & 502.12 & 508.65 & 505.39 \\
\hline \multicolumn{8}{|c|}{ FF2 } \\
\hline$\left[s_{1}, s_{2}\right]$ & SI & $F_{V}=\sqrt{2} F$ & $F_{V}=\sqrt{3} F$ & SD & Min & $\operatorname{Max}$ & Mean \\
\hline$\left[0.1296 \mathrm{GeV}^{2}, 1 \mathrm{GeV}^{2}\right]$ & 503.03 & 503.02 & 502.65 & 501.75 & 495.76 & 502.01 & $\overline{498.88}$ \\
\hline$\left[0.1296 \mathrm{GeV}^{2}, 2 \mathrm{GeV}^{2}\right]$ & 513.08 & 513.06 & 512.70 & 511.75 & 505.46 & 512.00 & 508.73 \\
\hline$\left[0.1296 \mathrm{GeV}^{2}, 3 \mathrm{GeV}^{2}\right]$ & 513.29 & 513.28 & 512.91 & 511.96 & 505.66 & 512.21 & 508.94 \\
\hline$\left[0.1296 \mathrm{GeV}^{2}, 3.125 \mathrm{GeV}^{2}\right]$ & 513.33 & 513.32 & 512.95 & 512.01 & 505.71 & 512.26 & 508.98 \\
\hline
\end{tabular}


TABLE VIII. IB-corrected $a_{\mu}^{\mathrm{HVP}, \mathrm{LO}}[\pi \pi, \tau]$ in units of $10^{-10}$ using the measured mass spectrum by ALEPH with $B_{\pi \pi}=(25.471 \pm 0.097 \pm 0.085) \%$. The rest is as in Table VII.

\begin{tabular}{|c|c|c|c|c|c|c|c|}
\hline \multicolumn{8}{|c|}{ FF1 } \\
\hline$\left[s_{1}, s_{2}\right]$ & SI & $F_{V}=\sqrt{2} F$ & $F_{V}=\sqrt{3} F$ & SD & Min & Max & Mean \\
\hline$\left[0.1296 \mathrm{GeV}^{2}, 1 \mathrm{GeV}^{2}\right]$ & 495.28 & 495.27 & 494.92 & 494.05 & 488.25 & 494.30 & 491.27 \\
\hline$\left[0.1296 \mathrm{GeV}^{2}, 2 \mathrm{GeV}^{2}\right]$ & 506.57 & 506.56 & 506.21 & 505.29 & 499.15 & 505.53 & 502.34 \\
\hline$\left[0.1296 \mathrm{GeV}^{2}, 3 \mathrm{GeV}^{2}\right]$ & 506.82 & 506.81 & 506.45 & 505.53 & 499.38 & 505.77 & 502.58 \\
\hline$\left[0.1296 \mathrm{GeV}^{2}, 3.125 \mathrm{GeV}^{2}\right]$ & 506.82 & 506.81 & 506.46 & 505.53 & 499.39 & 505.78 & 502.58 \\
\hline \multicolumn{8}{|c|}{$\mathrm{FF} 2$} \\
\hline$\left[s_{1}, s_{2}\right]$ & SI & $F_{V}=\sqrt{2} F$ & $F_{V}=\sqrt{3} F$ & SD & Min & $\operatorname{Max}$ & Mean \\
\hline$\left[0.1296 \mathrm{GeV}^{2}, 1 \mathrm{GeV}^{2}\right]$ & 498.86 & 498.85 & 498.50 & 497.63 & 491.81 & 497.87 & 494.84 \\
\hline$\left[0.1296 \mathrm{GeV}^{2}, 2 \mathrm{GeV}^{2}\right]$ & 510.16 & 510.15 & 509.80 & 508.87 & 502.72 & 509.12 & 505.92 \\
\hline$\left[0.1296 \mathrm{GeV}^{2}, 3 \mathrm{GeV}^{2}\right]$ & 510.41 & 510.40 & 510.04 & 509.12 & 502.95 & 509.36 & 506.16 \\
\hline$\left[0.1296 \mathrm{GeV}^{2}, 3.125 \mathrm{GeV}^{2}\right]$ & 510.41 & 510.40 & 510.05 & 509.12 & 502.96 & 509.36 & 506.16 \\
\hline
\end{tabular}

TABLE IX. IB-corrected $a_{\mu}^{\mathrm{HVP}, \mathrm{LO}}[\pi \pi, \tau]$ in units of $10^{-10}$ using the measured mass spectrum by CLEO with $B_{\pi \pi}=(25.36 \pm 0.44) \%$. The rest is as in Table VII.

\begin{tabular}{|c|c|c|c|c|c|c|c|}
\hline \multicolumn{8}{|c|}{ FF1 } \\
\hline$\left[s_{1}, s_{2}\right]$ & SI & $F_{V}=\sqrt{2} F$ & $F_{V}=\sqrt{3} F$ & SD & Min & Max & Mean \\
\hline$\left[0.1296 \mathrm{GeV}^{2}, 1 \mathrm{GeV}^{2}\right]$ & 498.51 & 498.50 & 498.14 & 497.27 & 491.43 & 497.52 & 494.47 \\
\hline$\left[0.1296 \mathrm{GeV}^{2}, 2 \mathrm{GeV}^{2}\right]$ & 508.98 & 508.97 & 508.61 & 507.69 & 501.54 & 507.93 & 504.74 \\
\hline$\left[0.1296 \mathrm{GeV}^{2}, 3 \mathrm{GeV}^{2}\right]$ & 509.15 & 509.14 & 508.79 & 507.86 & 501.70 & 508.11 & 504.91 \\
\hline$\left[0.1296 \mathrm{GeV}^{2}, 3.125 \mathrm{GeV}^{2}\right]$ & 509.20 & 509.18 & 508.83 & 507.91 & 501.75 & 508.15 & 504.95 \\
\hline \multicolumn{8}{|c|}{ FF2 } \\
\hline$\left[s_{1}, s_{2}\right]$ & SI & $F_{V}=\sqrt{2} F$ & $F_{V}=\sqrt{3} F$ & SD & Min & $\operatorname{Max}$ & Mean \\
\hline$\left[0.1296 \mathrm{GeV}^{2}, 1 \mathrm{GeV}^{2}\right]$ & 502.10 & 502.09 & 501.74 & 500.86 & 495.00 & 501.11 & 498.06 \\
\hline$\left[0.1296 \mathrm{GeV}^{2}, 2 \mathrm{GeV}^{2}\right]$ & 512.58 & 512.57 & 512.22 & 511.29 & 505.12 & 511.58 & 508.33 \\
\hline$\left[0.1296 \mathrm{GeV}^{2}, 3 \mathrm{GeV}^{2}\right]$ & 512.76 & 512.75 & 512.39 & 511.47 & 505.29 & 511.71 & 508.50 \\
\hline$\left[0.1296 \mathrm{GeV}^{2}, 3.125 \mathrm{GeV}^{2}\right]$ & 512.80 & 512.79 & 512.43 & 511.51 & 505.33 & 511.75 & 508.54 \\
\hline
\end{tabular}

TABLE X. IB-corrected $a_{\mu}^{\mathrm{HVP}, \mathrm{LO}}[\pi \pi, \tau]$ in units of $10^{-10}$ using the measured mass spectrum by OPAL with $B_{\pi \pi}=(25.46 \pm 0.17 \pm 0.29) \%$. The rest is as in Table VII.

FF1

\begin{tabular}{|c|c|c|c|c|c|c|c|}
\hline$\left[s_{1}, s_{2}\right]$ & SI & $F_{V}=\sqrt{2} F$ & $F_{V}=\sqrt{3} F$ & SD & Min & Max & $\overline{\text { Mean }}$ \\
\hline$\left[0.1296 \mathrm{GeV}^{2}, 1 \mathrm{GeV}^{2}\right]$ & 509.50 & 509.51 & 509.07 & 508.04 & 501.31 & 508.34 & 504.82 \\
\hline$\left[0.1296 \mathrm{GeV}^{2}, 2 \mathrm{GeV}^{2}\right]$ & 521.29 & 521.29 & 520.86 & 519.77 & 512.69 & 520.06 & 516.34 \\
\hline$\left[0.1296 \mathrm{GeV}^{2}, 3 \mathrm{GeV}^{2}\right]$ & 521.49 & 521.49 & 521.06 & 519.96 & 512.88 & 520.25 & 516.56 \\
\hline$\left[0.1296 \mathrm{GeV}^{2}, 3.125 \mathrm{GeV}^{2}\right]$ & 521.49 & 521.49 & 521.06 & 519.97 & 512.88 & 520.26 & 516.57 \\
\hline
\end{tabular}

FF2

\begin{tabular}{|c|c|c|c|c|c|c|c|}
\hline$\left[s_{1}, s_{2}\right]$ & SI & $F_{V}=\sqrt{2} F$ & $F_{V}=\sqrt{3} F$ & SD & Min & Max & Mean \\
\hline$\left[0.1296 \mathrm{GeV}^{2}, 1 \mathrm{GeV}^{2}\right]$ & 512.99 & 512.99 & 512.56 & 511.53 & 504.78 & 511.82 & 508.30 \\
\hline$\left[0.1296 \mathrm{GeV}^{2}, 2 \mathrm{GeV}^{2}\right]$ & 524.79 & 524.79 & 524.36 & 523.27 & 516.17 & 523.56 & 519.86 \\
\hline$\left[0.1296 \mathrm{GeV}^{2}, 3 \mathrm{GeV}^{2}\right]$ & 524.99 & 524.99 & 524.56 & 523.46 & 516.36 & 523.76 & 520.06 \\
\hline$\left[0.1296 \mathrm{GeV}^{2}, 3.125 \mathrm{GeV}^{2}\right]$ & 524.99 & 524.99 & 524.56 & 523.46 & 516.36 & 523.76 & 520.06 \\
\hline
\end{tabular}


TABLE XI. IB-corrected $a_{\mu}^{\mathrm{HVP}, \mathrm{LO}}[\pi \pi, \tau]$ in units of $10^{-10}$ at $\mathcal{O}\left(p^{4}\right)$. The first error is related to the systematic uncertainties on the mass spectrum and also includes contributions from the $\tau$-mass and $V_{u d}$ uncertainties. The second error arises from $B_{\pi \pi^{0}}$ and $B_{e}$, and the third error arises from the isospin-breaking corrections.

\begin{tabular}{lcrr}
\hline \hline & & $a_{\mu}^{\mathrm{HVP}, \mathrm{LO}}[\pi \pi, \tau]$ & \\
\hline Experiment & $2 m_{\pi^{ \pm}}-0.36 \mathrm{GeV}$ & $0.36-1.8 \mathrm{GeV}$ & $\mathrm{TOTAL}$ \\
\hline Belle & $8.81 \pm 0.00 \pm 0.14_{-0.34}^{+0.16}$ & $511.14 \pm 1.94 \pm 7.99_{-2.09}^{+1.91}$ & $519.95 \pm 1.94 \pm 7.99_{-2.12}^{+1.91}$ \\
ALEPH & $8.89 \pm 0.00 \pm 0.05_{-0.34}^{+0.16}$ & $508.26 \pm 4.48 \pm 2.82_{-2.09}^{+1.91}$ & $517.15 \pm 4.48 \pm 2.82_{-2.12}^{+1.91}$ \\
CLEO & $8.85 \pm 0.00 \pm 0.15_{-0.34}^{+0.16}$ & $510.63 \pm 3.40 \pm 8.93_{-2.08}^{+1.90}$ & $519.48 \pm 3.40 \pm 8.93_{-2.11}^{+1.90}$ \\
OPAL & $8.89 \pm 0.00 \pm 0.12_{-0.34}^{+0.15}$ & $522.81 \pm 10.04 \pm 7.00_{-2.12}^{+1.87}$ & $531.70 \pm 10.04 \pm 7.00_{-2.15}^{+1.87}$ \\
\hline \hline
\end{tabular}

TABLE XII. IB-corrected $a_{\mu}^{\mathrm{HVP}, \mathrm{LO}}[\pi \pi, \tau]$ in units of $10^{-10}$ at $\mathcal{O}\left(p^{6}\right)$. The rest is as in Table XI.

\begin{tabular}{lcrr}
\hline \hline & & $a_{\mu}^{\mathrm{HVP}, \mathrm{LO}}[\pi \pi, \tau]$ & \\
\hline Experiment & $2 m_{\pi^{ \pm}}-0.36 \mathrm{GeV}$ & $0.36-1.8 \mathrm{GeV}$ & $\mathrm{TOTAL}$ \\
\hline Belle & $7.77 \pm 0.00 \pm 0.12_{-0.59}^{+1.20}$ & $507.18 \pm 1.91 \pm 7.88_{-3.76}^{+4.72}$ & $514.95 \pm 1.91 \pm 7.88_{-3.81}^{+4.87}$ \\
ALEPH & $7.84 \pm 0.00 \pm 0.04_{-0.60}^{+1.21}$ & $504.37 \pm 4.35 \pm 2.79_{-3.70}^{+4.63}$ & $512.21 \pm 4.35 \pm 2.79_{-3.75}^{+4.78}$ \\
CLEO & $7.80 \pm 0.00 \pm 0.14_{-0.59}^{+1.21}$ & $506.74 \pm 3.28 \pm 8.84_{-3.71}^{+4.63}$ & $514.54 \pm 3.28 \pm 8.84_{-3.76}^{+4.78}$ \\
OPAL & $7.84 \pm 0.00 \pm 0.10_{-0.60}^{+1.20}$ & $518.32 \pm 9.69 \pm 6.92_{-4.12}^{+5.25}$ & $526.16 \pm 9.69 \pm 6.92_{-4.16}^{+5.39}$ \\
\hline \hline
\end{tabular}

$$
10^{10} \cdot a_{\mu}^{\mathrm{HVP},\left.\mathrm{LO}\right|_{\pi \pi, \text { data }}}=514.6 \pm 2.8_{\text {spectra }+\mathrm{BRs}_{-3.9 \mathrm{IB}}}^{+5.0}
$$

at $\mathcal{O}\left(p^{6}\right)$.

The IB errors come from the uncertainty on $\Gamma(\rho \rightarrow \pi \pi \gamma)$ (FF1 vs FF2) and either from the difference between the $F_{V}=\sqrt{2} F$ and $S D$ results [in Eq. (79)] or from the difference between the mean and minimum/maximum results [in Eq. (80)].

Contrary to previous estimates [62,67-69,77], the errors in $\left.a_{\mu}^{\mathrm{HVP}, \mathrm{LO}}\right|_{\pi \pi, \tau \mathrm{data}}$ happen to be dominated by the uncertainty on the IB contributions [but for the lower error on Eq. (79)].

When Eqs. (79) and (80) are supplemented with the fourpion tau decays measurements (up to $1.5 \mathrm{GeV}$ ) and with $e^{+} e^{-}$data at larger energies in these modes (and with $e^{+} e^{-}$ data in all other channels making up the hadronic cross section), we get $[7,69]$

$$
\begin{aligned}
10^{10} \cdot a_{\mu}^{\mathrm{HVP},\left.\mathrm{LO}\right|_{\tau \mathrm{data}}}= & 705.7 \pm 2.8_{\text {spectra }+\mathrm{BRs}_{-2.1 \mathrm{IB}}+1.9} \pm 2.0_{e^{+} e^{-}} \\
& \pm 0.1_{\text {narrowres }} \pm 0.7_{\mathrm{QCD}}
\end{aligned}
$$

at $\mathcal{O}\left(p^{4}\right)$ and

$$
\begin{aligned}
10^{10} \cdot a_{\mu}^{\mathrm{HVP},\left.\mathrm{LO}\right|_{\tau \mathrm{data}}}= & 700.7 \pm 2.8_{\text {spectra }+\mathrm{BRs}_{-3.9 \mathrm{IB}}+5.0} \pm 2.0_{e^{+} e^{-}} \\
& \pm 0.1_{\text {narrowres }} \pm 0.7_{\mathrm{QCD}}
\end{aligned}
$$

at $\mathcal{O}\left(p^{6}\right)$, and we have also included the uncertainties corresponding to using $e^{+} e^{-}$data for those contributions not covered by tau decay measurements and to the inclusion of narrow resonances and the perturbative QCD part.

Adding errors in quadrature, an uncertainty of ${ }_{-4.1}^{+4.0}\left({ }_{-5.2}^{+6.1}\right)$ is obtained at $\mathcal{O}\left(p^{4}\right)\left[\mathcal{O}\left(p^{6}\right)\right]$. These numbers (all in units of $10^{-10}$ ) have to be compared with the error of 4.0 in Ref. [2].

When all other (QED, EW, and subleading hadronic) contributions are added to Eqs. (81) and (82) according to Ref. [2], the 3.7 $\sigma$ [2] deficit of the SM prediction with respect to the BNL measurement [45] is reduced to

$$
\Delta a_{\mu} \equiv a_{\mu}^{\exp }-a_{\mu}^{\mathrm{SM}}=(15.3 \pm 7.7) \times 10^{-10}
$$

at $\mathcal{O}\left(p^{4}\right)$ and

$$
\Delta a_{\mu} \equiv a_{\mu}^{\exp }-a_{\mu}^{\mathrm{SM}}=\left(20.3_{-8.9}^{+8.3}\right) \times 10^{-10}
$$

at $\mathcal{O}\left(p^{6}\right)$, which are 2.0 and $2.3 \sigma$, respectively.

\section{CONCLUSIONS}

In this work, we have revisited the resonance chiral Lagrangian computation of the isospin-breaking and radiative corrections to the $\tau^{-} \rightarrow \pi^{-} \pi^{0} \nu_{\tau} \gamma$ decays in Ref. [62], by including the terms that start to contribute to the $\mathcal{O}\left(p^{6}\right)$ chiral LECs. Our main motivation for that was to revisit the determination of $a_{\mu}^{\mathrm{HVP}, \mathrm{LO}}$ using tau decay data so that it could-when combined with the $e^{+} e^{-}$measurementsreduce the Standard Model error on $a_{\mu}$, thus enhancing the sensitivity to new physics of the current BNL and future 
FNAL and Japan proton accelerator research complex measurements.

Our isospin-breaking corrections improve the agreement between $\tau$ and $e^{+} e^{-}$dipion data (both in the spectrum and its integral), which endorses our evaluation of $\left.a_{\mu}^{\mathrm{HVP}, \mathrm{LO}}\right|_{\tau \text { data }}$. Our main results are $a_{\mu}^{\mathrm{HVP},\left.\mathrm{LO}\right|_{\text {zdata }}}=\left(705.7_{-4.1}^{+4.0}\right) \times 10^{-10}$ (including the same contributions as in Ref. [62]) and $\left.a_{\mu}^{\mathrm{HVP}, \mathrm{LO}}\right|_{\text {zdata }}=\left(700.7_{-5.2}^{+6.1}\right) \times 10^{-10}$ [when the operators starting to contribute to the $\mathcal{O}\left(p^{6}\right)$, LECs are also considered]. These reduce the anomaly $\Delta a_{\mu} \equiv a_{\mu}^{\exp }-a_{\mu}^{\mathrm{SM}}$ to 2.0 and $2.3 \sigma$, respectively.

We also provide a detailed study of the $\pi \pi$ spectrum, $E_{\gamma}$ distribution, and branching ratio, for different cuts on the photon energy. These $\tau^{-} \rightarrow \pi^{-} \pi^{0} \nu_{\tau} \gamma$ decays observables have the potential to reduce drastically the error of our predictions, so we eagerly await their measurement at Belle-II.

\section{ACKNOWLEDGMENTS}

A. M. acknowledges Conacyt support through his Ph.D. scholarship. P. R. thanks the funding of Fondo SEPCinvestav 2018 (Project No. 142). A. M. and P. R. have benefitted from enriching discussions on this topic with Gabriel López Castro and Genaro Toledo Sánchez. We thank Vincenzo Cirigliano, Antonio Pich, and Jorge Portolés for helpful suggestions regarding the presentation and discussion of our results and Antonio Rojas, Eduard de la Cruz Burelo and Iván Heredia de la Cruz for their valuable help. We are indebted to Alex Keshavarzi, Bogdan Malaescu, Hisaki Hayashii, and Jorge Portolés for providing us with the BABAR, Belle, and OPAL datasets.

\section{APPENDIX A: FIT RESULTS}

Since the $\kappa_{i}^{V}$ couplings are related with the $\omega$ exchange, which is known to give an important contribution to the $\tau \rightarrow \pi \pi \gamma \nu_{\tau}$ decays, we perform a global fit using the relations for the resonance saturation of the anomalous sector LECs at NLO [88], Eqs. (10)-(18) in Sec. II E and the estimation of the LECs in Ref. [168].

Neglecting all the other contributions, we get

$$
\begin{gathered}
\kappa_{1}^{V}=(-2.1 \pm 0.7) \times 10^{-2} \mathrm{GeV}^{-1}, \\
\kappa_{2}^{V}=(-8.8 \pm 9.1) \times 10^{-3} \mathrm{GeV}^{-1}, \\
\kappa_{3}^{V}=(2.2 \pm 5.8) \times 10^{-3} \mathrm{GeV}^{-1}, \\
\kappa_{6}^{V}=(-2.1 \pm 0.3) \times 10^{-2} \mathrm{GeV}^{-1}, \\
\kappa_{7}^{V}=(1.2 \pm 0.5) \times 10^{-2} \mathrm{GeV}^{-1}, \\
\kappa_{8}^{V}=(3.1 \pm 0.9) \times 10^{-2} \mathrm{GeV}^{-1},
\end{gathered}
$$

$$
\begin{gathered}
\kappa_{9}^{V}=(-0.1 \pm 5.9) \times 10^{-3} \mathrm{GeV}^{-1}, \\
\kappa_{10}^{V}=(-5.9 \pm 9.6) \times 10^{-3} \mathrm{GeV}^{-1}, \\
\kappa_{11}^{V}=(-3.0 \pm 0.6) \times 10^{-2} \mathrm{GeV}^{-1}, \\
\kappa_{12}^{V}=(1.0 \pm 0.8) \times 10^{-2} \mathrm{GeV}^{-1}, \\
\kappa_{13}^{V}=(-5.3 \pm 1.1) \times 10^{-3} \mathrm{GeV}^{-1}, \\
\kappa_{18}^{V}=(4.7 \pm 0.8) \times 10^{-3} \mathrm{GeV}^{-1},
\end{gathered}
$$

These values are in good agreement with our earlier estimation in Sec. II E, $\left|\kappa_{i}^{V}\right| \lesssim 0.025 \mathrm{GeV}^{-1}$.

\section{APPENDIX B: KINEMATICS}

\section{1. $\tau^{-}(P) \rightarrow \pi^{-}\left(p_{-}\right) \pi^{0}\left(p_{0}\right) \gamma(k) \nu_{\tau}(q)$ kinematics}

To describe this type of decays, we need five independent variables. We choose $s=\left(p_{-}+p_{0}\right)^{2} ; u=\left(P-p_{-}\right)^{2}$; $x=(k+q)^{2} ; \theta_{\nu}$, which is the angle between the direction of the $\pi^{-} \pi^{0} \mathrm{c} . \mathrm{m}$. frame in the $\tau$ lepton rest frame and the direction of $\vec{q}$ in the $\pi^{-} \pi^{0}$ c.m. frame (see Fig. 19); and $\phi_{-}$, which is angle between the plane of the $\pi^{-} \pi^{0} \mathrm{c}$.m. frame and the plane of the $\gamma \nu_{\tau}$ c.m. frame. We can write the invariants in terms of these variables,

$$
\begin{gathered}
P \cdot p_{0}=\frac{s+u-x-m_{\pi^{0}}^{2}}{2}, \\
q \cdot k=\frac{x-M_{\gamma}^{2}}{2}, \\
p_{-} \cdot p_{0}=\frac{s-m_{\pi^{-}}^{2}-m_{\pi^{0}}^{2}}{2}, \\
p_{-} \cdot(q+k)=\frac{u-x-m_{\pi^{0}}^{2}}{2}, \\
P \cdot(q+k)=\frac{x-s+m_{\tau}^{2}}{2},
\end{gathered}
$$

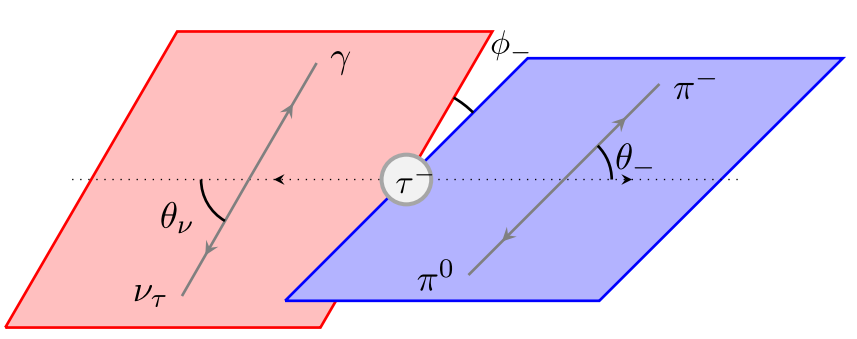

FIG. 19. The $\tau^{-} \rightarrow \pi^{-} \pi^{0} \gamma \nu_{\tau}$ decay in the $\tau$-lepton rest frame. 


$$
\begin{gathered}
P \cdot p_{-}=\frac{\left(m_{\pi^{-}}^{2}-m_{\pi^{0}}^{2}+s\right)\left(m_{\tau}^{2}+s-x\right)}{4 s}+\frac{\lambda^{1 / 2}\left(s, x, m_{\tau}^{2}\right) \lambda^{1 / 2}\left(m_{\tau}^{2}, m_{\pi^{-}}^{2}, m_{\pi^{0}}^{2}\right)}{4 s} \cos \theta_{-} \\
=\frac{m_{\tau}^{2}+m_{\pi^{-}}^{2}-u}{2}, \\
P \cdot k=\frac{\left(m_{\tau}^{2}-s+x\right)\left(x+M_{\gamma}^{2}\right)}{4 x}-\frac{\left(x-M_{\gamma}^{2}\right) \lambda^{1 / 2}\left(s, x, m_{\tau}^{2}\right)}{4 x} \cos \theta_{\nu}, \\
p_{-} \cdot k=\frac{\left(x+M_{\gamma}^{2}\right)\left(m_{\tau}^{2}-s-u+m_{\pi^{0}}^{2}\right)}{4 x}-\frac{\left(x-M_{\gamma}^{2}\right) \cos \theta_{\nu}}{4 x \lambda^{1 / 2}\left(s, x, m_{\tau}^{2}\right)} A(s, u, x) \\
-\frac{\left(x-M_{\gamma}^{2}\right) \lambda^{1 / 2}\left(s, m_{\pi^{-}}^{2}, m_{\pi^{0}}^{2}\right)}{4 \sqrt{x} \sqrt{s}} \sin \theta_{\nu} \sin \theta_{-} \cos \phi_{-}, \\
\epsilon^{\mu \nu \alpha \beta} k_{\mu} P_{\nu} p_{-\alpha} q_{\beta}=\frac{\left(x-M_{\gamma}^{2}\right) \lambda^{1 / 2}\left(s, m_{\pi^{-}}^{2}, m_{\pi^{0}}^{2}\right) \lambda^{1 / 2}\left(s, x, m_{\tau}^{2}\right)}{8 \sqrt{s} \sqrt{x}} \sin \theta_{\nu} \sin \theta_{-} \sin \phi_{-}, \\
\epsilon^{\mu \nu \alpha \beta} k_{\mu} P_{\nu} p_{-\alpha} p_{0 \beta}=\epsilon^{\mu \nu \alpha \beta} k_{\mu} P_{\nu} p_{0 \alpha} q_{\beta}=\epsilon^{\mu \nu \alpha \beta} k_{\mu} p_{-\nu} p_{0 \alpha} q_{\beta}=\epsilon^{\mu \nu \alpha \beta} P_{\mu} p_{-\nu} p_{0 \alpha} q_{\beta}=-\epsilon^{\mu \nu \alpha \beta} k_{\mu} P_{\nu} p_{-\alpha} q_{\beta},
\end{gathered}
$$

where

$$
A(s, u, x)=m_{\tau}^{4}+s(s+u)+x\left(u-s-2 m_{\pi^{-}}^{2}\right)+m_{\pi^{0}}^{2}\left(m_{\tau}^{2}-s+x\right)-m_{\tau}^{2}(2 s+u+x) .
$$

Working in the $\tau$-lepton rest frame, we have

$$
\begin{gathered}
E_{\gamma}=\frac{\left(m_{\tau}^{2}-s+x\right)\left(x+M_{\gamma}^{2}\right)}{4 m_{\tau} x}-\frac{\left(x-M_{\gamma}^{2}\right) \lambda^{1 / 2}\left(s, x, m_{\tau}^{2}\right)}{4 m_{\tau} x} \cos \theta_{\nu}, \\
E_{\nu}=|\vec{q}|=\frac{\left(m_{\tau}^{2}-s+x\right)\left(x-M_{\gamma}^{2}\right)}{4 m_{\tau} x}+\frac{\left(x-M_{\gamma}^{2}\right) \lambda^{1 / 2}\left(s, x, m_{\tau}^{2}\right)}{4 m_{\tau} x} \cos \theta_{\nu}, \\
\vec{k}=\left(-\frac{\left(x+M_{\gamma}^{2}\right) \lambda^{1 / 2}\left(s, x, m_{\tau}^{2}\right)}{4 m_{\tau} x}+\frac{\left(m_{\tau}^{2}-s+x\right)\left(x-M_{\gamma}^{2}\right)}{4 m_{\tau} x} \cos \theta_{\nu}\right) \hat{e}_{z}+\frac{x-M_{\gamma}^{2}}{2 \sqrt{x}} \sin \theta_{\nu} \hat{e}_{x}, \\
\vec{q}=\left(-\frac{\left(x-M_{\gamma}^{2}\right) \lambda^{1 / 2}\left(s, x, m_{\tau}^{2}\right)}{4 m_{\tau} x}-\frac{\left(m_{\tau}^{2}-s+x\right)\left(x-M_{\gamma}^{2}\right)}{4 m_{\tau} x} \cos \theta_{\nu}\right) \hat{e}_{z}-\frac{x-M_{\gamma}^{2}}{2 \sqrt{x}} \sin \theta_{\nu} \hat{e}_{x}, \\
E_{-}=\frac{\left(m_{\tau}^{2}+s-x\right)\left(s+m_{\pi^{-}}^{2}-m_{\pi^{0}}^{2}\right)}{4 m_{\tau} s}+\frac{\lambda^{1 / 2}\left(s, x, m_{\tau}^{2}\right) \lambda^{1 / 2}\left(s, m_{\pi^{-}}^{2}, m_{\pi^{0}}^{2}\right)}{4 m_{\tau} s} \cos \theta_{-} \\
=\frac{m_{\tau}^{2}+m_{\pi^{-}}^{2}-u}{2 m_{\tau}}, \\
E_{0}=\frac{\left(m_{\tau}^{2}+s-x\right)\left(s-m_{\pi^{-}}^{2}+m_{\pi^{0}}^{2}\right)}{4 m_{\tau} s}-\frac{\lambda^{1 / 2}\left(s, x, m_{\tau}^{2}\right) \lambda^{1 / 2}\left(s, m_{\pi^{-}}^{2}, m_{\pi^{0}}^{2}\right)}{4 m_{\tau} s} \cos \theta_{-} \\
=\frac{s+u-x-m_{\pi^{-}}^{2}}{2 m_{\tau}}, \\
\left|\vec{p}_{-}\right|=\frac{\lambda^{1 / 2}\left(u, m_{\tau}^{2}, m_{\pi^{-}}^{2}\right)}{2 m_{\tau}},
\end{gathered}
$$




$$
\begin{gathered}
\vec{p}_{-}=\left(\frac{\left(s+m_{\pi^{-}}^{2}-m_{\pi^{0}}^{2}\right) \lambda^{1 / 2}\left(s, x, m_{\tau}^{2}\right)}{4 m_{\tau} s}+\frac{\left(m_{\tau}^{2}+s-x\right) \lambda^{1 / 2}\left(s, m_{\pi^{-}}^{2}, m_{\pi^{0}}^{2}\right)}{4 m_{\tau} s} \cos \theta_{-}\right) \hat{e}_{z}+\frac{\lambda^{1 / 2}\left(s, m_{\pi^{-}}^{2}, m_{\pi^{0}}^{2}\right)}{2 \sqrt{s}} \sin \theta_{-} \hat{e}_{\rho}, \\
\vec{p}_{0}=\left(\frac{\left(s-m_{\pi^{-}}^{2}+m_{\pi^{0}}^{2}\right) \lambda^{1 / 2}\left(s, x, m_{\tau}^{2}\right)}{4 m_{\tau} s}-\frac{\left(m_{\tau}^{2}+s-x\right) \lambda^{1 / 2}\left(s, m_{\pi^{-}}^{2}, m_{\pi^{0}}^{2}\right)}{4 m_{\tau} s} \cos \theta_{-}\right) \hat{e}_{z}-\frac{\lambda^{1 / 2}\left(s, m_{\pi^{-}}^{2}, m_{\pi^{0}}^{2}\right)}{2 \sqrt{s}} \sin \theta_{-} \hat{e}_{\rho}, \\
\cos \theta_{-}=\frac{2 s\left(m_{\tau}^{2}+m_{\pi^{-}}^{2}-u\right)-\left(m_{\tau}^{2}+s-x\right)\left(s+m_{\pi^{-}}^{2}-m_{\pi^{0}}^{2}\right)}{\lambda^{1 / 2}\left(s, x, m_{\tau}^{2}\right) \lambda^{1 / 2}\left(s, m_{\pi^{-}}^{2}, m_{\pi^{0}}^{2}\right)}, \\
\cos \theta_{\nu}=\frac{\left(m_{\tau}^{2}-s+x\right)\left(x+M_{\gamma}^{2}\right)-4 m_{\tau} E_{\gamma} x}{\left(x-M_{\gamma}^{2}\right) \lambda^{1 / 2}\left(s, x, m_{\tau}^{2}\right)},
\end{gathered}
$$

where $\lambda(x, y, z)=x^{2}+y^{2}+z^{2}-2 x y-2 x z-2 y z$ is the Kallen function and $\hat{e}_{\rho}=\cos \phi_{-} \hat{e}_{x}+\sin \phi_{-} \hat{e}_{y}$. From Eq. (B17), we get

$$
x_{ \pm}(s, u)=\frac{-m_{\pi^{-}}^{4}+\left(m_{\pi^{0}}^{2}-s\right)\left(m_{\tau}^{2}-u\right)+m_{\pi^{-}}^{2}\left(m_{\tau}^{2}+m_{\pi^{0}}^{2}+s+u\right)}{2 m_{\pi^{-}}^{2}} \pm \frac{\lambda^{1 / 2}\left(u, m_{\tau}^{2}, m_{\pi^{-}}^{2}\right) \lambda^{1 / 2}\left(s, m_{\pi^{-}}^{2}, m_{\pi^{0}}^{2}\right)}{2 m_{\pi^{-}}^{2}}
$$

and

$$
u_{ \pm}(s, x)=m_{\tau}^{2}+m_{\pi^{-}}^{2}-\frac{\left(m_{\tau}^{2}+s-x\right)\left(s+m_{\pi^{-}}^{2}-m_{\pi^{0}}^{2}\right)}{2 s} \pm \frac{\lambda^{1 / 2}\left(s, x, m_{\tau}^{2}\right) \lambda^{1 / 2}\left(s, m_{\pi^{-}}^{2}, m_{\pi^{0}}^{2}\right)}{2 s}
$$

these bounds on $u$ and $x$ correspond to the forward and backward directions, i.e., by taking $\theta_{-}=0, \pi$. For the nonradiative decay, we have

$$
\mathcal{D}^{I I I}=\left\{u_{-}(s, 0) \leq u \leq u_{+}(s, 0),\left(m_{\pi^{-}}+m_{\pi^{0}}\right)^{2} \leq s \leq m_{\tau}^{2}\right\}
$$

this region is plotted in Fig. 20, which corresponds to the projection $\mathcal{R}^{I I I}$ onto the $s u$-plane. In the case of the radiative decay, we have

$$
\mathcal{D}^{I V}=\left\{x_{\min }(s, u) \leq x \leq x_{\max }(s, u), u_{\min }(s) \leq u \leq u_{\max }(s),\left(m_{\pi^{-}}+m_{\pi^{-}}\right)^{2} \leq s \leq\left(m_{\tau}-M_{\gamma}\right)^{2}\right\}
$$

with

$$
x_{\min }(s, u)=\left\{\begin{array}{lll}
x_{-}(s, u) & u_{+}\left(s, M_{\gamma}^{2}\right) \leq u \leq\left(m_{\tau}-m_{\pi^{-}}\right)^{2}, & \left(m_{\pi^{-}}+m_{\pi^{0}}\right)^{2} \leq s \leq s^{*} \\
M_{\gamma}^{2} & u_{-}\left(s, M_{\gamma}^{2}\right) \leq u \leq u_{+}\left(s, M_{\gamma}^{2}\right), & s^{*} \leq s \leq\left(m_{\tau}-M_{\gamma}\right)^{2}
\end{array}\right.
$$




$$
\begin{gathered}
x_{\max }(s, u)=x_{+}(s, u), \\
u_{\min }(s)=u_{-}\left(s, M_{\gamma}^{2}\right), \\
u_{\max }(s)= \begin{cases}\left(m_{\tau}-m_{\pi^{-}}\right)^{2} & \left(m_{\pi^{-}}+m_{\pi^{0}}\right)^{2} \leq s \leq s^{*}, \\
u_{+}\left(s, M_{\gamma}^{2}\right) & s^{*} \leq s \leq\left(m_{\tau}-M_{\gamma}\right)^{2},\end{cases}
\end{gathered}
$$

where $s^{*}=\frac{m_{\tau}\left(m_{\tau} m_{\pi^{-}}+m_{\pi^{0}}^{2}-m_{\pi^{-}}^{2}\right)-M_{\gamma}^{2} m_{\pi^{-}}}{m_{\tau}-m_{\pi^{-}}}$is the value that maximizes $u_{+}\left(s, M_{\gamma}^{2}\right)$. We will be working in the isospin limit $\left(m_{u}=m_{d}\right)$, i.e., $m_{\pi^{-}}^{2}=m_{\pi^{0}}^{2}$, and thus many of the last expressions will be simplified. We use a nonvanishing $M_{\gamma}$ in order to deal with the IR divergences; at the end, these divergences are canceled out by those divergences of the nonradiative decay so we can take the limit $M_{\gamma} \rightarrow 0$. The projection $\mathcal{R}^{I V}=\mathcal{R}^{I V / I I I} \cup \mathcal{R}^{I I I}$ of the $\mathcal{D}^{I V}$ is plotted in Fig. 20 for $M_{\gamma} \rightarrow 0$.

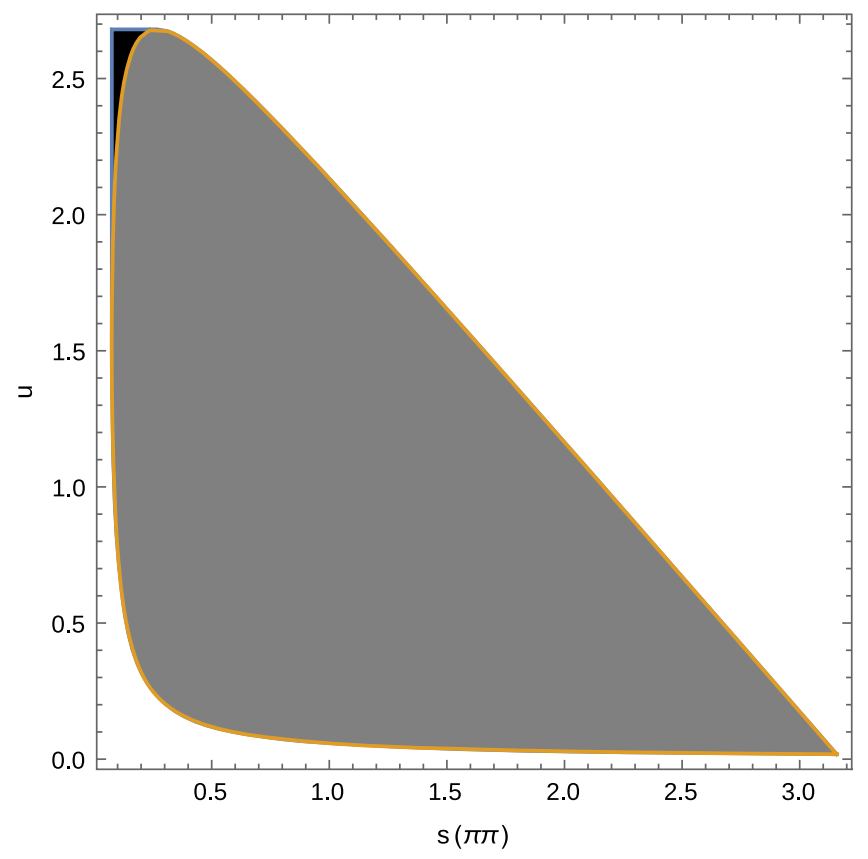

FIG. 20. Projection of the kinematic region for the nonradiative decay $\mathcal{R}^{I I I}$ (gray) and the radiative decay $\mathcal{R}^{I V}=\mathcal{R}^{I V / I I I} \cup \mathcal{R}^{I I I}$ (black and gray) onto the $s u$-plane. $\mathcal{R}^{I V / I I I}$ (black) is the kinematic region, which is only accessible to the radiative decay.

\section{APPENDIX C: VECTOR FORM FACTORS}

$$
\begin{aligned}
v_{1}^{R}= & \frac{1}{F^{2}}\left(\frac{16 k \cdot p_{0}\left(2 \kappa_{12}^{V}+\kappa_{16}^{V}\right)\left(-\left(2 k \cdot p_{-}+s\right)\left(2 \kappa_{12}^{V}+\kappa_{16}^{V}\right)+2(P-q)^{2} \kappa_{17}^{V}\right)}{D_{\omega}\left[\left(k+p_{0}\right)^{2}\right]}\right. \\
& +\frac{\sqrt{2} F_{V}}{M_{\rho}^{2}}\left(2 s \lambda_{12}^{V}-\left(4 k \cdot p_{0}+s\right)\left(\lambda_{13}^{V}+\lambda_{14}^{V}-\lambda_{15}^{V}\right)+\left(2 k \cdot p_{-}+s\right) \lambda_{16}^{V}-4 k \cdot p_{-} \lambda_{17}^{V}\right. \\
& \left.+4 k \cdot p_{-} \lambda_{18}^{V}+s \lambda_{18}^{V}+4 k \cdot p_{-} \lambda_{19}^{V}+2 s \lambda_{19}^{V}+4 k \cdot p_{0} \lambda_{21}^{V}+2 k \cdot p_{0} \lambda_{22}^{V}-2 k \cdot p_{-} \lambda_{22}^{V}\right) \\
& +\frac{1}{M_{a_{1}}^{2} D_{a_{1}}\left[\left(k+p_{-}\right)^{2}\right]}\left(-8\left(-2 k \cdot p_{0} M_{a_{1}}^{2} s+\left(k \cdot p_{-}-M_{a_{1}}^{2}\right) s^{2}+2\left(k \cdot p_{0}\right)\left(k \cdot p_{-}\right)\right.\right. \\
& \left.\times\left(2 M_{a_{1}}^{2}+s\right)\right)\left(\lambda_{12}^{A}\right)^{2}-8 k \cdot p_{-}\left(2 k \cdot p_{0}+s\right)\left(2 M_{a_{1}}^{2}+s\right)\left(\lambda_{13}^{A}\right)^{2}-2 \sqrt{2} F_{A}\left(k \cdot p_{0}\right) s \lambda_{15}^{A} \\
& -2 \sqrt{2} F_{A}\left(k \cdot p_{-}\right) s \lambda_{15}^{A}-\sqrt{2} F_{A} s^{2} \lambda_{15}^{A}-8 \sqrt{2} F_{A} k \cdot p_{-} M_{a_{1}}^{2} \lambda_{17}^{A}-4 \sqrt{2} F_{A}\left(k \cdot p_{-}\right) s \lambda_{17}^{A} \\
& +16\left(k \cdot p_{0}\right)\left(k \cdot p_{-}\right) s \lambda_{15}^{A} \lambda_{17}^{A}+16\left(k \cdot p_{-}\right)^{2} s \lambda_{15}^{A} \lambda_{17}^{A}+8\left(k \cdot p_{-}\right) s^{2} \lambda_{15}^{A} \lambda_{17}^{A} \\
& +32\left(k \cdot p_{-}\right)^{2} M_{a_{1}}^{2}\left(\lambda_{17}^{A}\right)^{2}+16\left(k \cdot p_{-}\right)^{2} s\left(\lambda_{17}^{A}\right)^{2}+\lambda_{13}^{A}\left(8\left(k \cdot p_{-}\right) s(P-q)^{2} \lambda_{15}^{A}\right. \\
& \left.+\left(2 k \cdot p_{0}-2 k \cdot p_{-}+s\right)\left(2 M_{a_{1}}^{2}+s\right)\left(\sqrt{2} F_{A}-8 k \cdot p_{-} \lambda_{17}^{A}\right)\right)+\lambda_{12}^{A}\left(-8\left(s \left(2 k \cdot p_{-}\left(M_{a_{1}}^{2}-s\right)\right.\right.\right. \\
& \left.\left.+M_{a_{1}}^{2} s\right)+2 k \cdot p_{0}\left(4 k \cdot p_{-} M_{a_{1}}^{2}-2\left(k \cdot p_{-}\right) s+M_{a_{1}}^{2} s\right)\right) \lambda_{13}^{A}-8\left(k \cdot p_{-}-M_{a_{1}}^{2}\right) s(P-q)^{2} \lambda_{15}^{A} \\
& \left.\left.\left.+\left(k \cdot p_{0}\left(4 M_{a_{1}}^{2}-2 s\right)-s\left(2 M_{a_{1}}^{2}+s\right)+k \cdot p_{-}\left(-4 M_{a_{1}}^{2}+2 s\right)\right)\left(\sqrt{2} F_{A}-8 k \cdot p_{-} \lambda_{17}^{A}\right)\right)\right)\right)
\end{aligned}
$$




$$
\begin{aligned}
& v_{1}^{R R}=\frac{1}{2 \sqrt{2} F^{2}}\left(\frac{64 k \cdot p_{0} F_{V}\left(-\left(2 k \cdot p_{-}+s\right)\left(2 \kappa_{12}^{V}+\kappa_{16}^{V}\right)+2(P-q)^{2} \kappa_{17}^{V}\right) \kappa_{3}^{V V}}{M_{\rho}^{2} D_{\omega}\left[\left(k+p_{0}\right)^{2}\right]}\right. \\
& -\frac{64 k \cdot p_{0}\left(4 k \cdot p_{0}+2 k \cdot p_{-}+s\right)\left(2 \kappa_{12}^{V}+\kappa_{16}^{V}\right) \kappa_{3}^{V V}\left(-F_{V}+2 \sqrt{2}(P-q)^{2} \lambda_{22}^{V}\right)}{D_{\rho}\left[(P-q)^{2}\right] D_{\omega}\left[\left(k+p_{0}\right)^{2}\right]} \\
& +\frac{2 F_{V}}{M_{a_{1}}^{2} M_{\rho}^{2} D_{a_{1}}\left[\left(k+p_{-}\right)^{2}\right]}\left(2 \left(2 \sqrt{2} F_{A} k \cdot p_{-} M_{a_{1}}^{2}-\sqrt{2} F_{A}\left(k \cdot p_{-}\right) s+\sqrt{2} F_{A} M_{a_{1}}^{2} s\right.\right. \\
& +4\left(-2 k \cdot p_{0} M_{a_{1}}^{2} s+\left(k \cdot p_{-}-M_{a_{1}}^{2}\right) s^{2}+2\left(k \cdot p_{0}\right)\left(k \cdot p_{-}\right)\left(2 M_{a_{1}}^{2}+s\right)\right) \lambda_{12}^{A} \\
& +4\left(2 k \cdot p_{0}+s\right)\left(2 k \cdot p_{-} M_{a_{1}}^{2}-\left(k \cdot p_{-}\right) s+M_{a_{1}}^{2} s\right) \lambda_{13}^{A}+8\left(k \cdot p_{0}\right)\left(k \cdot p_{-}\right) s \lambda_{15}^{A} \\
& +8\left(k \cdot p_{-}\right)^{2} s \lambda_{15}^{A}-8 k \cdot p_{0} M_{a_{1}}^{2} s \lambda_{15}^{A}-8 k \cdot p_{-} M_{a_{1}}^{2} s \lambda_{15}^{A}+4\left(k \cdot p_{-}\right) s^{2} \lambda_{15}^{A} \\
& \left.-4 M_{a_{1}}^{2} s^{2} \lambda_{15}^{A}-16\left(k \cdot p_{-}\right)^{2} M_{a_{1}}^{2} \lambda_{17}^{A}+8\left(k \cdot p_{-}\right)^{2} s \lambda_{17}^{A}-8 k \cdot p_{-} M_{a_{1}}^{2} s \lambda_{17}^{A}\right) \lambda_{2}^{V A} \\
& -k \cdot p_{-}\left(2 \sqrt{2} F_{A} M_{a_{1}}^{2}+\sqrt{2} F_{A} s+4\left(4 k \cdot p_{0} M_{a_{1}}^{2}-2\left(k \cdot p_{0}\right) s-s^{2}\right) \lambda_{12}^{A}\right. \\
& +4\left(2 k \cdot p_{0}+s\right)\left(2 M_{a_{1}}^{2}+s\right) \lambda_{13}^{A}-8\left(k \cdot p_{0}\right) s \lambda_{15}^{A}-8\left(k \cdot p_{-}\right) s \lambda_{15}^{A}-4 s^{2} \lambda_{15}^{A} \\
& \left.\left.-16 k \cdot p_{-} M_{a_{1}}^{2} \lambda_{17}^{A}-8\left(k \cdot p_{-}\right) s \lambda_{17}^{A}\right)\left(\lambda_{4}^{V A}+2 \lambda_{5}^{V A}\right)\right) \\
& +\frac{\left(\sqrt{2} F_{V}-4(P-q)^{2} \lambda_{22}^{V}\right)}{M_{a_{1}}^{2} D_{a_{1}}\left[\left(k+p_{-}\right)^{2}\right] D_{\rho}\left[(P-q)^{2}\right]}\left(-2\left(-4 \sqrt{2}\left(-2 k \cdot p_{0} M_{a_{1}}^{2} s+\left(k \cdot p_{-}-M_{a_{1}}^{2}\right) s^{2}\right.\right.\right. \\
& \left.+2\left(k \cdot p_{0}\right)\left(k \cdot p_{-}\right)\left(2 M_{a_{1}}^{2}+s\right)\right) \lambda_{12}^{A}+\left(4 k \cdot p_{0} M_{a_{1}}^{2}-2\left(k \cdot p_{0}\right) s-s^{2}\right) \\
& \left.\times\left(F_{A}-4 \sqrt{2} k \cdot p_{-} \lambda_{13}^{A}-4 \sqrt{2} k \cdot p_{-} \lambda_{17}^{A}\right)\right) \lambda_{2}^{V A}-2 s(P-q)^{2}\left(F_{A}+4 \sqrt{2}\left(k \cdot p_{-}-M_{a_{1}}^{2}\right) \lambda_{12}^{A}\right. \\
& \left.-4 \sqrt{2} k \cdot p_{-} \lambda_{13}^{A}-4 \sqrt{2} k \cdot p_{-} \lambda_{17}^{A}\right) \lambda_{3}^{V A}+\left(2 k \cdot p_{0}+s\right)\left(-4 \sqrt{2}\left(2 k \cdot p_{-} M_{a_{1}}^{2}-\left(k \cdot p_{-}\right) s\right.\right. \\
& \left.\left.\left.+M_{a_{1}}^{2} s\right) \lambda_{12}^{A}+\left(2 M_{a_{1}}^{2}+s\right)\left(F_{A}-4 \sqrt{2} k \cdot p_{-} \lambda_{13}^{A}-4 \sqrt{2} k \cdot p_{-} \lambda_{17}^{A}\right)\right)\left(\lambda_{4}^{V A}+2 \lambda_{5}^{V A}\right)\right) \\
& +\frac{2 F_{V}}{M_{\rho}^{2} D_{\rho}\left[(P-q)^{2}\right]}\left(-2(P-q)^{2} \lambda_{22}^{V}\left((P-q)^{2}-8 s \lambda_{2}^{V V}\right.\right. \\
& \left.-2\left(4 k \cdot p_{0}+s\right) \lambda_{3}^{V V}+8 k \cdot p_{0} \lambda_{4}^{V V}+2 s \lambda_{4}^{V V}-16 k \cdot p_{0} \lambda_{5}^{V V}-4 s \lambda_{5}^{V V}\right) \\
& \left.+\sqrt{2} F_{V}\left(-4 s \lambda_{2}^{V V}-\left(4 k \cdot p_{0}+s\right)\left(\lambda_{3}^{V V}-\lambda_{4}^{V V}+2 \lambda_{5}^{V V}\right)\right)\right) \\
& \left.-\frac{4 F_{V}\left(\sqrt{2}\left(4 k \cdot p_{0}+s\right) G_{V} \lambda_{7}^{V V}+s \lambda_{21}^{V}\left(s-2\left(4 k \cdot p_{0}+s\right) \lambda_{7}^{V V}\right)\right)}{M_{\rho}^{2} D_{\rho}[s]}\right)
\end{aligned}
$$

$$
\begin{aligned}
v_{1}^{R R R}= & -\frac{F_{V}\left(-\sqrt{2} F_{V}+4(P-q)^{2} \lambda_{22}^{V}\right)}{\sqrt{2} F^{2} M_{a_{1}}^{2} M_{\rho}^{2} D_{a_{1}}\left[\left(k+p_{-}\right)^{2}\right] D_{\rho}\left[(P-q)^{2}\right]} \\
& \times\left(-4\left(-2 k \cdot p_{0} M_{a_{1}}^{2} s+\left(k \cdot p_{-}-M_{a_{1}}^{2}\right) s^{2}+2\left(k \cdot p_{0}\right)\left(k \cdot p_{-}\right)\left(2 M_{a_{1}}^{2}+s\right)\right)\left(\lambda_{2}^{V A}\right)^{2}\right. \\
& -k \cdot p_{-}\left(\lambda_{4}^{V A}+2 \lambda_{5}^{V A}\right)\left(-2 s(P-q)^{2} \lambda_{3}^{V A}+\left(2 k \cdot p_{0}+s\right)\left(2 M_{a_{1}}^{2}+s\right)\left(\lambda_{4}^{V A}+2 \lambda_{5}^{V A}\right)\right) \\
& +2 \lambda_{2}^{V A}\left(2\left(k \cdot p_{-}-M_{a_{1}}^{2}\right) s(P-q)^{2} \lambda_{3}^{V A}+\left(s\left(2 k \cdot p_{-}\left(M_{a_{1}}^{2}-s\right)+M_{a_{1}}^{2} s\right)\right.\right. \\
& \left.\left.\left.+2 k \cdot p_{0}\left(4 k \cdot p_{-} M_{a_{1}}^{2}-2\left(k \cdot p_{-}\right) s+M_{a_{1}}^{2} s\right)\right)\left(\lambda_{4}^{V A}+2 \lambda_{5}^{V A}\right)\right)\right)
\end{aligned}
$$




$$
\begin{aligned}
v_{G I 1}^{R+R R}= & \frac{1}{F^{2} D_{\rho}\left[(P-q)^{2}\right] D_{\rho}[s]}\left(\sqrt { 2 } F _ { V } \left(2\left(M_{\rho}^{2}-s\right) s \lambda_{12}^{V}-\left(M_{\rho}^{2}-s\right)\left(4 k \cdot p_{0}+s\right) \lambda_{13}^{V}\right.\right. \\
& -4 k \cdot p_{0} M_{\rho}^{2} \lambda_{14}^{V}+4\left(k \cdot p_{0}\right) s \lambda_{14}^{V}-M_{\rho}^{2} s \lambda_{14}^{V}+s^{2} \lambda_{14}^{V}+4 k \cdot p_{0} M_{\rho}^{2} \lambda_{15}^{V}-4\left(k \cdot p_{0}\right) s \lambda_{15}^{V} \\
& +M_{\rho}^{2} s \lambda_{15}^{V}-s^{2} \lambda_{15}^{V}-2 k \cdot p_{0} M_{\rho}^{2} \lambda_{16}^{V}+2\left(k \cdot p_{0}\right) s \lambda_{16}^{V}+4 k \cdot p_{0} M_{\rho}^{2} \lambda_{17}^{V}-4\left(k \cdot p_{0}\right) s \lambda_{17}^{V} \\
& +2 M_{\rho}^{2} s \lambda_{17}^{V}-2 s^{2} \lambda_{17}^{V}-4 k \cdot p_{0} M_{\rho}^{2} \lambda_{18}^{V}+4\left(k \cdot p_{0}\right) s \lambda_{18}^{V}-M_{\rho}^{2} s \lambda_{18}^{V}+s^{2} \lambda_{18}^{V} \\
& -2 k \cdot p_{0} M_{\rho}^{2} \lambda_{19}^{V}+2\left(k \cdot p_{0}\right) s \lambda_{19}^{V}-4 k \cdot p_{-} M_{\rho}^{2} \lambda_{21}^{V}+4\left(k \cdot p_{-}\right) s \lambda_{21}^{V}-2 M_{\rho}^{2} s \lambda_{21}^{V} \\
& +s^{2} \lambda_{21}^{V}+6 k \cdot p_{0} M_{\rho}^{2} \lambda_{22}^{V}+2 k \cdot p_{-} M_{\rho}^{2} \lambda_{22}^{V}-6\left(k \cdot p_{0}\right) s \lambda_{22}^{V}-2\left(k \cdot p_{-}\right) s \lambda_{22}^{V} \\
& \left.+2 M_{\rho}^{2} s \lambda_{22}^{V}-2 s^{2} \lambda_{22}^{V}+8\left(k \cdot p_{0}\right) s \lambda_{21}^{V} \lambda_{7}^{V V}+2 s^{2} \lambda_{21}^{V} \lambda_{7}^{V V}\right)+2 G_{V}\left(\sqrt{2}\left(4 k \cdot p_{0}+s\right)\right. \\
& \times\left((P-q)^{2}-M_{\rho}^{2}\right) \lambda_{7}^{V}-\left(4 k \cdot p_{0}+s\right) F_{V} \lambda_{7}^{V V}+\sqrt{2} \lambda_{22}^{V}\left(-16\left(k \cdot p_{0}\right)^{2}-\left(2 M_{\rho}^{2}-s\right)\right. \\
& \left.\left.\times\left(2 k \cdot p_{-}+s\right)-2 k \cdot p_{0}\left(8 k \cdot p_{-}-2 M_{\rho}^{2}+3 s\right)+2\left(4 k \cdot p_{0}+s\right)(P-q)^{2} \lambda_{7}^{V V}\right)\right)
\end{aligned}
$$

$$
\begin{aligned}
& -4\left(s\left(4 k \cdot p_{0}+s\right)\left((P-q)^{2}-M_{\rho}^{2}\right) \lambda_{7}^{V} \lambda_{21}^{V}+\lambda_{22}^{V}\left(2\left(M_{\rho}^{2}-s\right) s(P-q)^{2} \lambda_{12}^{V}\right.\right. \\
& -\left(M_{\rho}^{2}-s\right)\left(4 k \cdot p_{0}+s\right)(P-q)^{2} \lambda_{13}^{V}-8\left(k \cdot p_{0}\right)^{2} M_{\rho}^{2} \lambda_{14}^{V}-8\left(k \cdot p_{0}\right)\left(k \cdot p_{-}\right) M_{\rho}^{2} \lambda_{14}^{V} \\
& +8\left(k \cdot p_{0}\right)^{2} s \lambda_{14}^{V}+8\left(k \cdot p_{0}\right)\left(k \cdot p_{-}\right) s \lambda_{14}^{V}-6 k \cdot p_{0} M_{\rho}^{2} s \lambda_{14}^{V}-2 k \cdot p_{-} M_{\rho}^{2} s \lambda_{14}^{V} \\
& +6\left(k \cdot p_{0}\right) s^{2} \lambda_{14}^{V}+2\left(k \cdot p_{-}\right) s^{2} \lambda_{14}^{V}-M_{\rho}^{2} s^{2} \lambda_{14}^{V}+s^{3} \lambda_{14}^{V}+8\left(k \cdot p_{0}\right)^{2} M_{\rho}^{2} \lambda_{15}^{V} \\
& +8\left(k \cdot p_{0}\right)\left(k \cdot p_{-}\right) M_{\rho}^{2} \lambda_{15}^{V}-8\left(k \cdot p_{0}\right)^{2} s \lambda_{15}^{V}-8\left(k \cdot p_{0}\right)\left(k \cdot p_{-}\right) s \lambda_{15}^{V} \\
& +6 k \cdot p_{0} M_{\rho}^{2} s \lambda_{15}^{V}+2 k \cdot p_{-} M_{\rho}^{2} s \lambda_{15}^{V}-6\left(k \cdot p_{0}\right) s^{2} \lambda_{15}^{V}-2\left(k \cdot p_{-}\right) s^{2} \lambda_{15}^{V}+M_{\rho}^{2} s^{2} \lambda_{15}^{V} \\
& -s^{3} \lambda_{15}^{V}-4\left(k \cdot p_{0}\right)^{2} M_{\rho}^{2} \lambda_{16}^{V}-4\left(k \cdot p_{0}\right)\left(k \cdot p_{-}\right) M_{\rho}^{2} \lambda_{16}^{V}+4\left(k \cdot p_{0}\right)^{2} s \lambda_{16}^{V} \\
& +4\left(k \cdot p_{0}\right)\left(k \cdot p_{-}\right) s \lambda_{16}^{V}-2 k \cdot p_{0} M_{\rho}^{2} s \lambda_{16}^{V}+2\left(k \cdot p_{0}\right) s^{2} \lambda_{16}^{V}+8\left(k \cdot p_{0}\right)^{2} M_{\rho}^{2} \lambda_{17}^{V} \\
& +8\left(k \cdot p_{0}\right)\left(k \cdot p_{-}\right) M_{\rho}^{2} \lambda_{17}^{V}-8\left(k \cdot p_{0}\right)^{2} s \lambda_{17}^{V}-8\left(k \cdot p_{0}\right)\left(k \cdot p_{-}\right) s \lambda_{17}^{V} \\
& +8 k \cdot p_{0} M_{\rho}^{2} s \lambda_{17}^{V}+4 k \cdot p_{-} M_{\rho}^{2} s \lambda_{17}^{V}-8\left(k \cdot p_{0}\right) s^{2} \lambda_{17}^{V}-4\left(k \cdot p_{-}\right) s^{2} \lambda_{17}^{V} \\
& +2 M_{\rho}^{2} s^{2} \lambda_{17}^{V}-2 s^{3} \lambda_{17}^{V}-8\left(k \cdot p_{0}\right)^{2} M_{\rho}^{2} \lambda_{18}^{V}-8\left(k \cdot p_{0}\right)\left(k \cdot p_{-}\right) M_{\rho}^{2} \lambda_{18}^{V} \\
& +8\left(k \cdot p_{0}\right)^{2} s \lambda_{18}^{V}+8\left(k \cdot p_{0}\right)\left(k \cdot p_{-}\right) s \lambda_{18}^{V}-6 k \cdot p_{0} M_{\rho}^{2} s \lambda_{18}^{V}-2 k \cdot p_{-} M_{\rho}^{2} s \lambda_{18}^{V} \\
& +6\left(k \cdot p_{0}\right) s^{2} \lambda_{18}^{V}+2\left(k \cdot p_{-}\right) s^{2} \lambda_{18}^{V}-M_{\rho}^{2} s^{2} \lambda_{18}^{V}+s^{3} \lambda_{18}^{V}-4\left(k \cdot p_{0}\right)^{2} M_{\rho}^{2} \lambda_{19}^{V} \\
& -4\left(k \cdot p_{0}\right)\left(k \cdot p_{-}\right) M_{\rho}^{2} \lambda_{19}^{V}+4\left(k \cdot p_{0}\right)^{2} s \lambda_{19}^{V}+4\left(k \cdot p_{0}\right)\left(k \cdot p_{-}\right) s \lambda_{19}^{V} \\
& -2 k \cdot p_{0} M_{\rho}^{2} s \lambda_{19}^{V}+2\left(k \cdot p_{0}\right) s^{2} \lambda_{19}^{V}-8\left(k \cdot p_{0}\right)\left(k \cdot p_{-}\right) M_{\rho}^{2} \lambda_{21}^{V}-8\left(k \cdot p_{-}\right)^{2} M_{\rho}^{2} \lambda_{21}^{V} \\
& -16\left(k \cdot p_{0}\right)^{2} s \lambda_{21}^{V}-8\left(k \cdot p_{0}\right)\left(k \cdot p_{-}\right) s \lambda_{21}^{V}+8\left(k \cdot p_{-}\right)^{2} s \lambda_{21}^{V}+4 k \cdot p_{0} M_{\rho}^{2} s \lambda_{21}^{V} \\
& -8 k \cdot p_{-} M_{\rho}^{2} s \lambda_{21}^{V}-6\left(k \cdot p_{0}\right) s^{2} \lambda_{21}^{V}+6\left(k \cdot p_{-}\right) s^{2} \lambda_{21}^{V}-2 M_{\rho}^{2} s^{2} \lambda_{21}^{V}+s^{3} \lambda_{21}^{V} \\
& +8\left(k \cdot p_{0}\right)^{2} M_{\rho}^{2} \lambda_{22}^{V}+8\left(k \cdot p_{0}\right)\left(k \cdot p_{-}\right) M_{\rho}^{2} \lambda_{22}^{V}-8\left(k \cdot p_{0}\right)^{2} s \lambda_{22}^{V} \\
& -8\left(k \cdot p_{0}\right)\left(k \cdot p_{-}\right) s \lambda_{22}^{V}+6 k \cdot p_{0} M_{\rho}^{2} s \lambda_{22}^{V}+2 k \cdot p_{-} M_{\rho}^{2} s \lambda_{22}^{V}-6\left(k \cdot p_{0}\right) s^{2} \lambda_{22}^{V} \\
& -2\left(k \cdot p_{-}\right) s^{2} \lambda_{22}^{V}+M_{\rho}^{2} s^{2} \lambda_{22}^{V}-s^{3} \lambda_{22}^{V}+16\left(k \cdot p_{0}\right)^{2} s \lambda_{21}^{V} \lambda_{7}^{V V} \\
& \left.\left.\left.+16\left(k \cdot p_{0}\right)\left(k \cdot p_{-}\right) s \lambda_{21}^{V} \lambda_{7}^{V V}+12\left(k \cdot p_{0}\right) s^{2} \lambda_{21}^{V} \lambda_{7}^{V V}+4\left(k \cdot p_{-}\right) s^{2} \lambda_{21}^{V} \lambda_{7}^{V V}+2 s^{3} \lambda_{21}^{V} \lambda_{7}^{V V}\right)\right)\right)
\end{aligned}
$$




$$
\begin{aligned}
& v_{2}^{R}=\frac{1}{F^{2}}\left(\frac { 8 ( 2 k \cdot p _ { - } + s ) } { M _ { \omega } ^ { 2 } D _ { \omega } [ ( k + p _ { 0 } ) ^ { 2 } ] } \left(-4\left(k \cdot p_{0}-M_{\omega}^{2}\right)\left(2 k \cdot p_{-}+s\right)\left(\kappa_{12}^{V}\right)^{2}+k \cdot p_{0} \kappa_{16}^{V}\right.\right. \\
& \times\left(\left(4 k \cdot p_{0}+2 k \cdot p_{-}-2 M_{\omega}^{2}+s\right) \kappa_{16}^{V}-2(P-q)^{2} \kappa_{17}^{V}\right)+\kappa_{12}^{V}\left(\left(-8\left(k \cdot p_{0}\right)^{2}+4 k \cdot p_{0} M_{\omega}^{2}\right.\right. \\
& \left.\left.\left.+2 M_{\omega}^{2}\left(2 k \cdot p_{-}+s\right)\right) \kappa_{16}^{V}+4\left(k \cdot p_{0}-M_{\omega}^{2}\right)(P-q)^{2} \kappa_{17}^{V}\right)\right)+\frac{\sqrt{2} F_{V}}{M_{\rho}^{2}}\left(2 s \lambda_{12}^{V}-s \lambda_{13}^{V}\right. \\
& +4 k \cdot p_{-} \lambda_{14}^{V}+s \lambda_{14}^{V}-4 k \cdot p_{-} \lambda_{15}^{V}-s \lambda_{15}^{V}-4 k \cdot p_{-} \lambda_{17}^{V}+2 k \cdot p_{-} \lambda_{18}^{V}-4 k \cdot p_{-} \lambda_{21}^{V} \\
& \left.+2 k \cdot p_{0} \lambda_{22}^{V}-2 k \cdot p_{-} \lambda_{22}^{V}\right)+\frac{2 \sqrt{2}}{M_{a_{1}}^{2} D_{a_{1}}\left[\left(k+p_{-}\right)^{2}\right]}\left(4 \sqrt{2}\left(k \cdot p_{-}\right)^{2}\left(2 k \cdot p_{0}+2 M_{a_{1}}^{2}+s\right)\right. \\
& \times\left(\lambda_{12}^{A}\right)^{2}+\lambda_{12}^{A}\left(4 \sqrt { 2 } k \cdot p _ { - } \left(-4\left(k \cdot p_{0}\right)\left(k \cdot p_{-}\right)+2 k \cdot p_{0} M_{a_{1}}^{2}+2 k \cdot p_{-} M_{a_{1}}^{2}-2\left(k \cdot p_{-}\right) s\right.\right. \\
& \left.+M_{a_{1}}^{2} s\right) \lambda_{13}^{A}+4 \sqrt{2}\left(k \cdot p_{-}\right)^{2}(P-q)^{2} \lambda_{15}^{A}+\left(-2\left(k \cdot p_{0}\right)\left(k \cdot p_{-}\right)+2\left(k \cdot p_{-}\right)^{2}\right. \\
& \left.\left.+2 k \cdot p_{0} M_{a_{1}}^{2}+2 k \cdot p_{-} M_{a_{1}}^{2}-\left(k \cdot p_{-}\right) s+M_{a_{1}}^{2} s\right)\left(-F_{A}+4 \sqrt{2} k \cdot p_{-} \lambda_{17}^{A}\right)\right) \\
& -\left(k \cdot p_{-}-M_{a_{1}}^{2}\right)\left(-4 \sqrt{2} k \cdot p_{-}\left(2 k \cdot p_{0}+s\right)\left(\lambda_{13}^{A}\right)^{2}-4 k \cdot p_{-} \lambda_{17}^{A}\left(F_{A}-2 \sqrt{2} k \cdot p_{-} \lambda_{17}^{A}\right)\right. \\
& +(P-q)^{2} \lambda_{15}^{A}\left(-F_{A}+4 \sqrt{2} k \cdot p_{-} \lambda_{17}^{A}\right)+\lambda_{13}^{A}\left(4 \sqrt{2} k \cdot p_{-}(P-q)^{2} \lambda_{15}^{A}\right. \\
& \left.\left.\left.\left.+\left(2 k \cdot p_{0}-2 k \cdot p_{-}+s\right)\left(F_{A}-4 \sqrt{2} k \cdot p_{-} \lambda_{17}^{A}\right)\right)\right)\right)\right) \\
& v_{2}^{R R}=\frac{1}{\sqrt{2} F^{2}}\left(\frac { 8 F _ { V } ( 2 k \cdot p _ { - } + s ) } { M _ { \rho } ^ { 2 } M _ { \omega } ^ { 2 } D _ { \omega } [ ( k + p _ { 0 } ) ^ { 2 } ] } \left(2(P-q)^{2} \kappa_{17}^{V}\left(-M_{\omega}^{2} \kappa_{3}^{V V}+\left(2 k \cdot p_{0}-M_{\omega}^{2}\right) \kappa_{4}^{V V}\right)\right.\right. \\
& +\kappa_{16}^{V}\left(M_{\omega}^{2}\left(2 k \cdot p_{-}+s\right) \kappa_{3}^{V V}-\left(2 k \cdot p_{0}-M_{\omega}^{2}\right)\left(4 k \cdot p_{0}+2 k \cdot p_{-}+s\right) \kappa_{4}^{V V}\right) \\
& \left.+2\left(2 k \cdot p_{-}+s\right) \kappa_{12}^{V}\left(M_{\omega}^{2} \kappa_{3}^{V V}+D_{\omega}\left[\left(k+p_{0}\right)^{2}\right] \kappa_{4}^{V V}\right)\right) \\
& +\frac{16\left(2 k \cdot p_{-}+s\right)\left(-F_{V}+2 \sqrt{2}(P-q)^{2} \lambda_{22}^{V}\right)}{M_{\omega}^{2} D_{\rho}\left[(P-q)^{2}\right] D_{\omega}\left[\left(k+p_{0}\right)^{2}\right]}\left(k \cdot p _ { 0 } \kappa _ { 1 6 } ^ { V } \left(\left((P-q)^{2}+M_{\omega}^{2}\right) \kappa_{3}^{V V}\right.\right. \\
& \left.+\left(2 k \cdot p_{0}-M_{\omega}^{2}\right) \kappa_{4}^{V V}\right)+\kappa_{12}^{V}\left(\left(-4\left(k \cdot p_{0}\right)^{2}+2 M_{\omega}^{2}\left(2 k \cdot p_{-}+s\right)\right.\right. \\
& \left.\left.\left.-2 k \cdot p_{0}\left(2 k \cdot p_{-}-3 M_{\omega}^{2}+s\right)\right) \kappa_{3}^{V V}+2 k \cdot p_{0}\left(-2 k \cdot p_{0}+M_{\omega}^{2}\right) \kappa_{4}^{V V}\right)\right) \\
& -\frac{2 k \cdot p_{-} F_{V}}{M_{a_{1}}^{2} M_{\rho}^{2} D_{a_{1}}\left[\left(k+p_{-}\right)^{2}\right]}\left(2 k \cdot p _ { - } \left(-\sqrt{2} F_{A}+4\left(2 k \cdot p_{0}+2 M_{a_{1}}^{2}+s\right) \lambda_{12}^{A}\right.\right. \\
& \left.-4\left(2 k \cdot p_{0}+s\right) \lambda_{13}^{A}+8 k \cdot p_{0} \lambda_{15}^{A}+8 k \cdot p_{-} \lambda_{15}^{A}+4 s \lambda_{15}^{A}+8 k \cdot p_{-} \lambda_{17}^{A}\right) \lambda_{2}^{V A} \\
& -\left(-4\left(2 k \cdot p_{0}\left(k \cdot p_{-}-M_{a_{1}}^{2}\right)-M_{a_{1}}^{2} s+k \cdot p_{-}\left(-2 M_{a_{1}}^{2}+s\right)\right) \lambda_{12}^{A}+\left(k \cdot p_{-}-M_{a_{1}}^{2}\right)\right. \\
& \left.\left.\times\left(\sqrt{2} F_{A}+4\left(2 k \cdot p_{0}+s\right) \lambda_{13}^{A}-4(P-q)^{2} \lambda_{15}^{A}-8 k \cdot p_{-} \lambda_{17}^{A}\right)\right)\left(\lambda_{4}^{V A}+2 \lambda_{5}^{V A}\right)\right) \\
& -\frac{\left(\sqrt{2} F_{V}-4(P-q)^{2} \lambda_{22}^{V}\right)}{M_{a_{1}}^{2} D_{a_{1}}\left[\left(k+p_{-}\right)^{2}\right] D_{\rho}\left[(P-q)^{2}\right]}\left(2 \left(4 \sqrt{2}\left(k \cdot p_{-}\right)^{2}\left(2 k \cdot p_{0}+2 M_{a_{1}}^{2}+s\right) \lambda_{12}^{A}\right.\right. \\
& +\left(2 k \cdot p_{0}\left(k \cdot p_{-}-M_{a_{1}}^{2}\right)-M_{a_{1}}^{2} s+k \cdot p_{-}\left(-2 M_{a_{1}}^{2}+s\right)\right)\left(F_{A}-4 \sqrt{2} k \cdot p_{-} \lambda_{13}^{A}\right. \\
& \left.\left.-4 \sqrt{2} k \cdot p_{-} \lambda_{17}^{A}\right)\right) \lambda_{2}^{V A}-\left(4 \sqrt{2}\left(k \cdot p_{-}\right)^{2} \lambda_{12}^{A}+\left(k \cdot p_{-}-M_{a_{1}}^{2}\right)\left(F_{A}-4 \sqrt{2} k \cdot p_{-} \lambda_{13}^{A}\right.\right. \\
& \left.\left.\left.-4 \sqrt{2} k \cdot p_{-} \lambda_{17}^{A}\right)\right)\left(2(P-q)^{2} \lambda_{3}^{V A}-\left(2 k \cdot p_{0}+s\right)\left(\lambda_{4}^{V A}+2 \lambda_{5}^{V A}\right)\right)\right) \\
& +\frac{F_{V}}{M_{\rho}^{2} D_{\rho}\left[(P-q)^{2}\right]}\left(\sqrt{2} F_{V}\left(-4 s \lambda_{2}^{V V}+\left(4 k \cdot p_{-}+s\right)\left(\lambda_{3}^{V V}-\lambda_{4}^{V V}\right)-2 s \lambda_{5}^{V V}\right)\right. \\
& \left.+2(P-q)^{2} \lambda_{22}^{V}\left((P-q)^{2}+8 s \lambda_{2}^{V V}-2\left(4 k \cdot p_{-}+s\right)\left(\lambda_{3}^{V V}-\lambda_{4}^{V V}\right)+4 s \lambda_{5}^{V V}\right)\right) \\
& \left.+\frac{2 F_{V}\left(\sqrt{2}\left(4 k \cdot p_{-}+s\right) G_{V} \lambda_{7}^{V V}+s \lambda_{21}^{V}\left(s-2\left(4 k \cdot p_{-}+s\right) \lambda_{7}^{V V}\right)\right)}{M_{\rho}^{2} D_{\rho}[s]}\right)
\end{aligned}
$$




$$
\begin{aligned}
& v_{2}^{R R R}=-\frac{\sqrt{2} k \cdot p_{-} F_{V}\left(-\sqrt{2} F_{V}+4(P-q)^{2} \lambda_{22}\right)}{F^{2} M_{a_{1}}^{2} M_{\rho}^{2} D_{a_{1}}\left[\left(k+p_{-}\right)^{2}\right] D_{\rho}\left[(P-q)^{2}\right]}\left(4 k \cdot p_{-}\left(2 k \cdot p_{0}+2 M_{a_{1}}^{2}+s\right)\left(\lambda_{2}^{V A}\right)^{2}\right. \\
& -\left(k \cdot p_{-}-M_{a_{1}}^{2}\right)\left(\lambda_{4}^{V A}+2 \lambda_{5}^{V A}\right)\left(2(P-q)^{2} \lambda_{3}^{V A}-\left(2 k \cdot p_{0}+s\right)\left(\lambda_{4}^{V A}+2 \lambda_{5}^{V A}\right)\right) \\
& -2 \lambda_{2}^{V A}\left(2 k \cdot p_{-}(P-q)^{2} \lambda_{3}^{V A}+\left(-4\left(k \cdot p_{0}\right)\left(k \cdot p_{-}\right)+2 k \cdot p_{0} M_{a_{1}}^{2}\right.\right. \\
& \left.\left.\left.+2 k \cdot p_{-} M_{a_{1}}^{2}-2\left(k \cdot p_{-}\right) s+M_{a_{1}}^{2} s\right)\left(\lambda_{4}^{V A}+2 \lambda_{5}^{V A}\right)\right)\right) \\
& v_{G I 2}^{R+R R}=\frac{\sqrt{2} F_{V}}{F^{2} D_{\rho}\left[(P-q)^{2}\right] D_{\rho}[s]}\left(2\left(M_{\rho}^{2}-s\right) s \lambda_{12}^{V}+s\left(-M_{\rho}^{2}+s\right) \lambda_{13}^{V}+4 k \cdot p_{-} M_{\rho}^{2} \lambda_{14}^{V}\right. \\
& -4\left(k \cdot p_{-}\right) s \lambda_{14}^{V}+M_{\rho}^{2} s \lambda_{14}^{V}-s^{2} \lambda_{14}^{V}-4 k \cdot p_{-} M_{\rho}^{2} \lambda_{15}^{V}+4\left(k \cdot p_{-}\right) s \lambda_{15}^{V}-M_{\rho}^{2} s \lambda_{15}^{V} \\
& +s^{2} \lambda_{15}^{V}-2 k \cdot p_{0} M_{\rho}^{2} \lambda_{16}^{V}-2 k \cdot p_{-} M_{\rho}^{2} \lambda_{16}^{V}+2\left(k \cdot p_{0}\right) s \lambda_{16}^{V}+2\left(k \cdot p_{-}\right) s \lambda_{16}^{V} \\
& -M_{\rho}^{2} s \lambda_{16}^{V}+s^{2} \lambda_{16}^{V}+4 k \cdot p_{0} M_{\rho}^{2} \lambda_{17}^{V}-4\left(k \cdot p_{0}\right) s \lambda_{17}^{V}+2 M_{\rho}^{2} s \lambda_{17}^{V}-2 s^{2} \lambda_{17}^{V} \\
& +2 k \cdot p_{-} M_{\rho}^{2} \lambda_{18}^{V}-2\left(k \cdot p_{-}\right) s \lambda_{18}^{V}+2 k \cdot p_{0} M_{\rho}^{2} \lambda_{19}^{V}+2 k \cdot p_{-} M_{\rho}^{2} \lambda_{19}^{V}-2\left(k \cdot p_{0}\right) s \lambda_{19}^{V} \\
& -2\left(k \cdot p_{-}\right) s \lambda_{19}^{V}+M_{\rho}^{2} s \lambda_{19}^{V}-s^{2} \lambda_{19}^{V}-4 k \cdot p_{-} M_{\rho}^{2} \lambda_{21}^{V}+4\left(k \cdot p_{-}\right) s \lambda_{21}^{V}+s^{2} \lambda_{21}^{V} \\
& +6 k \cdot p_{0} M_{\rho}^{2} \lambda_{22}^{V}+2 k \cdot p_{-} M_{\rho}^{2} \lambda_{22}^{V}-6\left(k \cdot p_{0}\right) s \lambda_{22}^{V}-2\left(k \cdot p_{-}\right) s \lambda_{22}^{V}+2 M_{\rho}^{2} s \lambda_{22}^{V} \\
& \left.-2 s^{2} \lambda_{22}^{V}-8\left(k \cdot p_{-}\right) s \lambda_{21}^{V} \lambda_{7}^{V V}-2 s^{2} \lambda_{21}^{V} \lambda_{7}^{V V}\right)-2 G_{V}\left(\sqrt{2}\left(4 k \cdot p_{-}+s\right)\right. \\
& \times\left((P-q)^{2}-M_{\rho}^{2}\right) \lambda_{7}^{V}-\left(4 k \cdot p_{-}+s\right) F_{V} \lambda_{7}^{V V}+\sqrt{2} \lambda_{22}^{V}\left(-16\left(k \cdot p_{-}\right)^{2}+8 k \cdot p_{-} M_{\rho}^{2}\right. \\
& \left.\left.-10\left(k \cdot p_{-}\right) s-s^{2}-2 k \cdot p_{0}\left(8 k \cdot p_{-}+s\right)+2(P-q)^{2}\left(4 k \cdot p_{-}+s\right) \lambda_{7}^{V V}\right)\right) \\
& +4\left(s\left(4 k \cdot p_{-}+s\right)\left((P-q)^{2}-M_{\rho}^{2}\right) \lambda_{7}^{V} \lambda_{21}^{V}+\lambda_{22}^{V}\left(-2\left(M_{\rho}^{2}-s\right) s(P-q)^{2} \lambda_{12}^{V}\right.\right. \\
& +\left(M_{\rho}^{2}-s\right) s(P-q)^{2} \lambda_{13}^{V}-8\left(k \cdot p_{0}\right)\left(k \cdot p_{-}\right) M_{\rho}^{2} \lambda_{14}^{V}-8\left(k \cdot p_{-}\right)^{2} M_{\rho}^{2} \lambda_{14}^{V} \\
& +8\left(k \cdot p_{0}\right)\left(k \cdot p_{-}\right) s \lambda_{14}^{V}+8\left(k \cdot p_{-}\right)^{2} s \lambda_{14}^{V}-2 k \cdot p_{0} M_{\rho}^{2} s \lambda_{14}^{V}-6 k \cdot p_{-} M_{\rho}^{2} s \lambda_{14}^{V} \\
& +2\left(k \cdot p_{0}\right) s^{2} \lambda_{14}^{V}+6\left(k \cdot p_{-}\right) s^{2} \lambda_{14}^{V}-M_{\rho}^{2} s^{2} \lambda_{14}^{V}+s^{3} \lambda_{14}^{V}+8\left(k \cdot p_{0}\right)\left(k \cdot p_{-}\right) M_{\rho}^{2} \lambda_{15}^{V} \\
& +8\left(k \cdot p_{-}\right)^{2} M_{\rho}^{2} \lambda_{15}^{V}-8\left(k \cdot p_{0}\right)\left(k \cdot p_{-}\right) s \lambda_{15}^{V}-8\left(k \cdot p_{-}\right)^{2} s \lambda_{15}^{V}+2 k \cdot p_{0} M_{\rho}^{2} s \lambda_{15}^{V} \\
& +6 k \cdot p_{-} M_{\rho}^{2} s \lambda_{15}^{V}-2\left(k \cdot p_{0}\right) s^{2} \lambda_{15}^{V}-6\left(k \cdot p_{-}\right) s^{2} \lambda_{15}^{V}+M_{\rho}^{2} s^{2} \lambda_{15}^{V}-s^{3} \lambda_{15}^{V} \\
& +4\left(k \cdot p_{0}\right)^{2} M_{\rho}^{2} \lambda_{16}^{V}+8\left(k \cdot p_{0}\right)\left(k \cdot p_{-}\right) M_{\rho}^{2} \lambda_{16}^{V}+4\left(k \cdot p_{-}\right)^{2} M_{\rho}^{2} \lambda_{16}^{V}-4\left(k \cdot p_{0}\right)^{2} s \lambda_{16}^{V} \\
& -8\left(k \cdot p_{0}\right)\left(k \cdot p_{-}\right) s \lambda_{16}^{V}-4\left(k \cdot p_{-}\right)^{2} s \lambda_{16}^{V}+4 k \cdot p_{0} M_{\rho}^{2} s \lambda_{16}^{V}+4 k \cdot p_{-} M_{\rho}^{2} s \lambda_{16}^{V} \\
& -4\left(k \cdot p_{0}\right) s^{2} \lambda_{16}^{V}-4\left(k \cdot p_{-}\right) s^{2} \lambda_{16}^{V}+M_{\rho}^{2} s^{2} \lambda_{16}^{V}-s^{3} \lambda_{16}^{V}-8\left(k \cdot p_{0}\right)^{2} M_{\rho}^{2} \lambda_{17}^{V} \\
& -8\left(k \cdot p_{0}\right)\left(k \cdot p_{-}\right) M_{\rho}^{2} \lambda_{17}^{V}+8\left(k \cdot p_{0}\right)^{2} s \lambda_{17}^{V}+8\left(k \cdot p_{0}\right)\left(k \cdot p_{-}\right) s \lambda_{17}^{V} \\
& -8 k \cdot p_{0} M_{\rho}^{2} s \lambda_{17}^{V}-4 k \cdot p_{-} M_{\rho}^{2} s \lambda_{17}^{V}+8\left(k \cdot p_{0}\right) s^{2} \lambda_{17}^{V}+4\left(k \cdot p_{-}\right) s^{2} \lambda_{17}^{V} \\
& -2 M_{\rho}^{2} s^{2} \lambda_{17}^{V}+2 s^{3} \lambda_{17}^{V}-4\left(k \cdot p_{0}\right)\left(k \cdot p_{-}\right) M_{\rho}^{2} \lambda_{18}^{V}-4\left(k \cdot p_{-}\right)^{2} M_{\rho}^{2} \lambda_{18}^{V} \\
& +4\left(k \cdot p_{0}\right)\left(k \cdot p_{-}\right) s \lambda_{18}^{V}+4\left(k \cdot p_{-}\right)^{2} s \lambda_{18}^{V}-2 k \cdot p_{-} M_{\rho}^{2} s \lambda_{18}^{V}+2\left(k \cdot p_{-}\right) s^{2} \lambda_{18}^{V} \\
& -4\left(k \cdot p_{0}\right)^{2} M_{\rho}^{2} \lambda_{19}^{V}-8\left(k \cdot p_{0}\right)\left(k \cdot p_{-}\right) M_{\rho}^{2} \lambda_{19}^{V}-4\left(k \cdot p_{-}\right)^{2} M_{\rho}^{2} \lambda_{19}^{V}+4\left(k \cdot p_{0}\right)^{2} s \lambda_{19}^{V} \\
& +8\left(k \cdot p_{0}\right)\left(k \cdot p_{-}\right) s \lambda_{19}^{V}+4\left(k \cdot p_{-}\right)^{2} s \lambda_{19}^{V}-4 k \cdot p_{0} M_{\rho}^{2} s \lambda_{19}^{V}-4 k \cdot p_{-} M_{\rho}^{2} s \lambda_{19}^{V} \\
& +4\left(k \cdot p_{0}\right) s^{2} \lambda_{19}^{V}+4\left(k \cdot p_{-}\right) s^{2} \lambda_{19}^{V}-M_{\rho}^{2} s^{2} \lambda_{19}^{V}+s^{3} \lambda_{19}^{V}+8\left(k \cdot p_{0}\right)\left(k \cdot p_{-}\right) M_{\rho}^{2} \lambda_{21}^{V} \\
& +8\left(k \cdot p_{-}\right)^{2} M_{\rho}^{2} \lambda_{21}^{V}-24\left(k \cdot p_{0}\right)\left(k \cdot p_{-}\right) s \lambda_{21}^{V}-24\left(k \cdot p_{-}\right)^{2} s \lambda_{21}^{V}+12 k \cdot p_{-} M_{\rho}^{2} s \lambda_{21}^{V} \\
& -2\left(k \cdot p_{0}\right) s^{2} \lambda_{21}^{V}-14\left(k \cdot p_{-}\right) s^{2} \lambda_{21}^{V}-s^{3} \lambda_{21}^{V}-8\left(k \cdot p_{0}\right)^{2} M_{\rho}^{2} \lambda_{22}^{V} \\
& -8\left(k \cdot p_{0}\right)\left(k \cdot p_{-}\right) M_{\rho}^{2} \lambda_{22}^{V}+8\left(k \cdot p_{0}\right)^{2} s \lambda_{22}^{V}+8\left(k \cdot p_{0}\right)\left(k \cdot p_{-}\right) s \lambda_{22}^{V} \\
& -6 k \cdot p_{0} M_{\rho}^{2} s \lambda_{22}^{V}-2 k \cdot p_{-} M_{\rho}^{2} s \lambda_{22}^{V}+6\left(k \cdot p_{0}\right) s^{2} \lambda_{22}^{V}+2\left(k \cdot p_{-}\right) s^{2} \lambda_{22}^{V}-M_{\rho}^{2} s^{2} \lambda_{22}^{V} \\
& +s^{3} \lambda_{22}^{V}+16\left(k \cdot p_{0}\right)\left(k \cdot p_{-}\right) s \lambda_{21}^{V} \lambda_{7}^{V V}+16\left(k \cdot p_{-}\right)^{2} s \lambda_{21}^{V} \lambda_{7}^{V V}+4\left(k \cdot p_{0}\right) s^{2} \lambda_{21}^{V} \lambda_{7}^{V V} \\
& \left.\left.+12\left(k \cdot p_{-}\right) s^{2} \lambda_{21}^{V} \lambda_{7}^{V V}+2 s^{3} \lambda_{21}^{V} \lambda_{7}^{V V}\right)\right)
\end{aligned}
$$




$$
\begin{aligned}
v_{3}^{R}= & \frac{2}{F^{2}}\left(\frac { 8 } { M _ { \omega } ^ { 2 } D _ { \omega } [ ( k + p _ { 0 } ) ^ { 2 } ] } \left(-4\left(4\left(k \cdot p_{0}\right)^{2}-M_{\omega}^{2}\left(2 k \cdot p_{-}+s\right)+k \cdot p_{0}\right.\right.\right. \\
& \left.\times\left(2 k \cdot p_{-}-2 M_{\omega}^{2}+s\right)\right)\left(\kappa_{12}^{V}\right)^{2}+k \cdot p_{0} \kappa_{16}^{V}\left(\left(2 k \cdot p_{-}+s\right) \kappa_{16}^{V}-2(P-q)^{2} \kappa_{17}^{V}\right) \\
& \left.+2 \kappa_{12}^{V}\left(\left(4\left(k \cdot p_{0}\right)^{2}-2 k \cdot p_{0} M_{\omega}^{2}+M_{\omega}^{2}\left(2 k \cdot p_{-}+s\right)\right) \kappa_{16}^{V}+2\left(k \cdot p_{0}-M_{\omega}^{2}\right)(P-q)^{2} \kappa_{17}^{V}\right)\right) \\
& +\frac{\sqrt{2} F_{V}\left(2 \lambda_{13}^{V}+\lambda_{16}^{V}+\lambda_{18}^{V}+2 \lambda_{19}^{V}\right)}{M_{\rho}^{2}} \\
& +\frac{8}{M_{a_{1}}^{2} D_{a_{1}}\left[\left(k+p_{-}\right)^{2}\right]}\left(\left(-2\left(k \cdot p_{0}\right)\left(k \cdot p_{-}\right)+2 k \cdot p_{0} M_{a_{1}}^{2}+2 k \cdot p_{-} M_{a_{1}}^{2}-\left(k \cdot p_{-}\right) s\right.\right. \\
& \left.+M_{a_{1}}^{2} s\right)\left(\lambda_{12}^{A}\right)^{2}-8 k \cdot p_{-}\left(2 k \cdot p_{0}+s\right)\left(\lambda_{13}^{A}\right)^{2}-2 \sqrt{2} F_{A} k \cdot p_{0} \lambda_{15}^{A}-2 \sqrt{2} F_{A} k \cdot p_{-} \lambda_{15}^{A} \\
& -\sqrt{2} F_{A} s \lambda_{15}^{A}-4 \sqrt{2} F_{A} k \cdot p_{-} \lambda_{17}^{A}+16\left(k \cdot p_{0}\right)\left(k \cdot p_{-}\right) \lambda_{15}^{A} \lambda_{17}^{A}+16\left(k \cdot p_{-}\right)^{2} \lambda_{15}^{A} \lambda_{17}^{A} \\
& +8\left(k \cdot p_{-}\right) s \lambda_{15}^{A} \lambda_{17}^{A}+16\left(k \cdot p_{-}\right)^{2}\left(\lambda_{17}^{A}\right)^{2}+\lambda_{13}^{A}\left(8 k \cdot p_{-}(P-q)^{2} \lambda_{15}^{A}+\left(2 k \cdot p_{0}\right.\right. \\
& \left.\left.-2 k \cdot p_{-}+s\right)\left(\sqrt{2} F_{A}-8 k \cdot p_{-} \lambda_{17}^{A}\right)\right)+\lambda_{12}^{A}\left(8 \left(k \cdot p_{0}\left(4 k \cdot p_{-}-2 M_{a_{1}}^{2}\right)-M_{a_{1}}^{2} s\right.\right. \\
& \left.+2 k \cdot p_{-}\left(M_{a_{1}}^{2}+s\right)\right) \lambda_{13}^{A}-8\left(k \cdot p_{-}-M_{a_{1}}^{2}\right)(P-q)^{2} \lambda_{15}^{A}+\left(2 k \cdot p_{0}-2 k \cdot p_{-}+4 M_{a_{1}}^{2}\right. \\
& \left.\left.\left.+s)\left(-\sqrt{2} F_{A}+8 k \cdot p_{-} \lambda_{17}^{A}\right)\right)\right)\right)
\end{aligned}
$$

$$
\begin{aligned}
v_{3}^{R R}= & \frac{1}{\sqrt{2} F^{2}}\left(-\frac{16 F_{V}}{M_{\rho}^{2} M_{\omega}^{2} D_{\omega}\left[\left(k+p_{0}\right)^{2}\right]}\left(2 \kappa _ { 1 2 } ^ { V } \left(-M_{\omega}^{2}\left(2 k \cdot p_{-}+s\right) \kappa_{3}^{V V}+\left(2 k \cdot p_{0}-M_{\omega}^{2}\right)\right.\right.\right. \\
& \left.\times\left(4 k \cdot p_{0}+2 k \cdot p_{-}+s\right) \kappa_{4}^{V V}\right)-\left(\left(2 k \cdot p_{-}+s\right) \kappa_{16}^{V}-2(P-q)^{2} \kappa_{17}^{V}\right)\left(M_{\omega}^{2} \kappa_{3}^{V V}\right. \\
& \left.\left.+D_{\omega}\left[\left(k+p_{0}\right)^{2}\right] \kappa_{4}^{V V}\right)\right)+\frac{32\left(-F_{V}+2 \sqrt{2}(P-q)^{2} \lambda_{22}^{V}\right)}{M_{\omega}^{2} D_{\rho}\left[(P-q)^{2}\right] D_{\omega}\left[\left(k+p_{0}\right)^{2}\right]}\left(-2 \kappa_{12}^{V}\left(\left(2\left(k \cdot p_{0}\right)^{2}\right.\right.\right. \\
& \left.\left.-M_{\omega}^{2}\left(2 k \cdot p_{-}+s\right)+k \cdot p_{0}\left(2 k \cdot p_{-}-3 M_{\omega}^{2}+s\right)\right) \kappa_{3}^{V V}+k \cdot p_{0}\left(-2 k \cdot p_{0}+M_{\omega}^{2}\right) \kappa_{4}^{V V}\right) \\
& \left.+k \cdot p_{0} \kappa_{16}^{V}\left(\left((P-q)^{2}+M_{\omega}^{2}\right) \kappa_{3}^{V V}+D_{\omega}\left[\left(k+p_{0}\right)^{2}\right] \kappa_{4}^{V V}\right)\right) \\
& +\frac{2 F_{V}}{M_{a_{1}}^{2} M_{\rho}^{2} D_{a_{1}}\left[\left(k+p_{-}\right)^{2}\right]}\left(-2\left(-4\left(2 k \cdot p_{0}\left(k \cdot p_{-}-M_{a_{1}}^{2}\right)-M_{a_{1}}^{2} s+k \cdot p_{-}\right.\right.\right. \\
& \left.\times\left(-2 M_{a_{1}}^{2}+s\right)\right) \lambda_{12}^{A}+\left(k \cdot p_{-}-M_{a_{1}}^{2}\right)\left(\sqrt{2} F_{A}+4\left(2 k \cdot p_{0}+s\right) \lambda_{13}^{A}-4(P-q)^{2} \lambda_{15}^{A}\right. \\
& \left.\left.-8 k \cdot p_{-} \lambda_{17}^{A}\right)\right) \lambda_{2}^{V A}+k \cdot p_{-}\left(-\sqrt{2} F_{A}+4\left(2 k \cdot p_{0}+2 M_{a_{1}}^{2}+s\right) \lambda_{12}^{A}-4\left(2 k \cdot p_{0}+s\right) \lambda_{13}^{A}\right. \\
& \left.\left.+8 k \cdot p_{0} \lambda_{15}^{A}+8 k \cdot p_{-} \lambda_{15}^{A}+4 s \lambda_{15}^{A}+8 k \cdot p_{-} \lambda_{17}^{A}\right)\left(\lambda_{4}^{V A}+2 \lambda_{5}^{V A}\right)\right) \\
& +\frac{\left(-\sqrt{2} F_{V}+4(P-q)^{2} \lambda_{22}^{V}\right)}{M_{a_{1}}^{2} D_{a_{1}}\left[\left(k+p_{-}\right)^{2}\right] D_{\rho}\left[(P-q)^{2}\right]}\left(-2\left(4 \sqrt { 2 } \left(2 k \cdot p_{0}\left(k \cdot p_{-}-M_{a_{1}}^{2}\right)-M_{a_{1}}^{2} s\right.\right.\right. \\
& \left.+k \cdot p_{-}\left(-2 M_{a_{1}}^{2}+s\right)\right) \lambda_{12}^{A}+\left(2 k \cdot p_{0}+2 M_{a_{1}}^{2}+s\right)\left(F_{A}-4 \sqrt{2} k \cdot p_{-} \lambda_{13}^{A}\right. \\
& \left.\left.-4 \sqrt{2} k \cdot p_{-} \lambda_{17}^{A}\right)\right) \lambda_{2}^{V A}+\left(F_{A}+4 \sqrt{2}\left(k \cdot p_{-}-M_{a_{1}}^{2}\right) \lambda_{12}^{A}-4 \sqrt{2} k \cdot p_{-} \lambda_{13}^{A}\right. \\
& \left.\left.-4 \sqrt{2} k \cdot p_{-} \lambda_{17}^{A}\right)\left(2(P-q)^{2} \lambda_{3}^{V A}-\left(2 k \cdot p_{0}+s\right)\left(\lambda_{4}^{V A}+2 \lambda_{5}^{V A}\right)\right)\right) \\
& +\frac{8 F_{V}\left(\sqrt{2} F_{V}-4(P-q)^{2} \lambda_{22}^{V}\right) \lambda_{5}^{V V}}{M_{\rho}^{2} D_{\rho}\left[(P-q)^{2}\right]}
\end{aligned}
$$




$$
\begin{aligned}
& v_{3}^{R R R}=-\frac{\sqrt{2} F_{V}\left(-\sqrt{2} F_{V}+4(P-q)^{2} \lambda_{22}^{V}\right)}{F^{2} M_{a_{1}}^{2} M_{\rho}^{2} D_{a_{1}}\left[\left(k+p_{-}\right)^{2}\right] D_{\rho}\left[(P-q)^{2}\right]}\left(-4\left(2 k \cdot p_{0}\left(k \cdot p_{-}-M_{a_{1}}^{2}\right)\right.\right. \\
& \left.-M_{a_{1}}^{2} s+k \cdot p_{-}\left(-2 M_{a_{1}}^{2}+s\right)\right)\left(\lambda_{2}^{V A}\right)^{2}+k \cdot p_{-}\left(\lambda_{4}^{V A}+2 \lambda_{5}^{V A}\right)\left(2(P-q)^{2} \lambda_{3}^{V A}\right. \\
& \left.-\left(2 k \cdot p_{0}+s\right)\left(\lambda_{4}^{V A}+2 \lambda_{5}^{V A}\right)\right)+2 \lambda_{2}^{V A}\left(2\left(k \cdot p_{-}-M_{a_{1}}^{2}\right)(P-q)^{2} \lambda_{3}^{V A}\right. \\
& \left.\left.-\left(k \cdot p_{0}\left(4 k \cdot p_{-}-2 M_{a_{1}}^{2}\right)-M_{a_{1}}^{2} s+2 k \cdot p_{-}\left(M_{a_{1}}^{2}+s\right)\right)\left(\lambda_{4}^{V A}+2 \lambda_{5}^{V A}\right)\right)\right) \\
& v_{G I 3}^{R+R R}=\frac{2 \sqrt{2}}{F^{2} D_{\rho}\left[(P-q)^{2}\right]}\left(F_{V}-2 \sqrt{2}(P-q)^{2} \lambda_{22}^{V}\right)\left(2 \lambda_{13}^{V}+\lambda_{16}^{V}+\lambda_{18}^{V}+\lambda_{19}^{V}\right) \\
& v_{4}^{R}=\frac{2}{F^{2}}\left(\frac { 8 } { M _ { \omega } ^ { 2 } D _ { \omega } [ ( k + p _ { 0 } ) ^ { 2 } ] } \left(4\left(k \cdot p_{0}-M_{\omega}^{2}\right)\left(2 k \cdot p_{-}+s\right)\left(\kappa_{12}^{V}\right)^{2}\right.\right. \\
& +k \cdot p_{0} \kappa_{16}^{V}\left(-\left(2 k \cdot p_{-}+s\right) \kappa_{16}^{V}+2\left(2 k \cdot p_{-}+M_{\omega}^{2}+s\right) \kappa_{17}^{V}\right)-2 \kappa_{12}^{V}\left(M_{\omega}^{2}\left(2 k \cdot p_{-}+s\right) \kappa_{16}^{V}\right. \\
& \left.\left.+2\left(-M_{\omega}^{2}\left(2 k \cdot p_{-}+s\right)+k \cdot p_{0}\left(2 k \cdot p_{-}-M_{\omega}^{2}+s\right)\right) \kappa_{17}^{V}\right)\right) \\
& \left.-\frac{\sqrt{2} F_{V}\left(\lambda_{13}^{V}+\lambda_{14}^{V}-\lambda_{15}^{V}-\lambda_{21}^{V}\right)}{M_{\rho}^{2}}-\frac{\sqrt{2}\left(2 \lambda_{12}^{A}+\lambda_{15}^{A}\right)\left(-F_{A}+4 \sqrt{2} k \cdot p_{-}\left(\lambda_{12}^{A}+\lambda_{13}^{A}+\lambda_{17}^{A}\right)\right)}{D_{a_{1}}\left[\left(k+p_{-}\right)^{2}\right]}\right) \\
& v_{4}^{R R}=\frac{\sqrt{2}}{F^{2}}\left(-\frac{8 F_{V}}{M_{\rho}^{2} M_{\omega}^{2} D_{\omega}\left[\left(k+p_{0}\right)^{2}\right]}\left(\left(2 k \cdot p_{-}+s\right) \kappa_{16}^{V}\left(M_{\omega}^{2} \kappa_{3}^{V V}+\left(-2 k \cdot p_{0}+M_{\omega}^{2}\right) \kappa_{4}^{V V}\right)\right.\right. \\
& -2 \kappa_{17}^{V}\left(M_{\omega}^{2}(P-q)^{2} \kappa_{3}^{V V}-\left(2 k \cdot p_{0}-M_{\omega}^{2}\right)\left(2 k \cdot p_{-}+s\right) \kappa_{4}^{V V}\right) \\
& \left.+2\left(2 k \cdot p_{-}+s\right) \kappa_{12}^{V}\left(M_{\omega}^{2} \kappa_{3}^{V V}+D_{\omega}\left[\left(k+p_{0}\right)^{2}\right] \kappa_{4}^{V V}\right)\right)-\frac{16 \kappa_{3}^{V V}\left(-F_{V}+2 \sqrt{2}(P-q)^{2} \lambda_{22}^{V}\right)}{M_{\omega}^{2} D_{\rho}\left[(P-q)^{2}\right] D_{\omega}\left[\left(k+p_{0}\right)^{2}\right]} \\
& \times\left(\left(2 M_{\omega}^{2}\left(2 k \cdot p_{-}+s\right)-2 k \cdot p_{0}\left(2 k \cdot p_{-}-2 M_{\omega}^{2}+s\right)\right) \kappa_{12}^{V}+k \cdot p_{0}\left(2 k \cdot p_{-}+2 M_{\omega}^{2}+s\right) \kappa_{16}^{V}\right) \\
& +\frac{\left(\sqrt{2} F_{V}-4(P-q)^{2} \lambda_{22}^{V}\right)\left(-F_{A}+4 \sqrt{2} k \cdot p_{-}\left(\lambda_{12}^{A}+\lambda_{13}^{A}+\lambda_{17}^{A}\right)\right)\left(2 \lambda_{2}^{V A}-\lambda_{3}^{V A}\right)}{D_{a_{1}}\left[\left(k+p_{-}\right)^{2}\right] D_{\rho}\left[(P-q)^{2}\right]} \\
& +\frac{4 k \cdot p_{-} F_{V}\left(2 \lambda_{12}^{A}+\lambda_{15}^{A}\right)\left(2 \lambda_{2}^{V A}-\lambda_{4}^{V A}-2 \lambda_{5}^{V A}\right)}{M_{\rho}^{2} D_{a_{1}}\left[\left(k+p_{-}\right)^{2}\right]}-\frac{2 F_{V}\left(\sqrt{2} G_{V}-2 s \lambda_{21}^{V}\right) \lambda_{7}^{V V}}{M_{\rho}^{2} D_{\rho}[s]} \\
& \left.-\frac{F_{V}\left(\sqrt{2} F_{V}\left(\lambda_{3}^{V V}-\lambda_{4}^{V V}+2 \lambda_{5}^{V V}\right)-2(P-q)^{2} \lambda_{22}^{V}\left(-1+2 \lambda_{3}^{V V}-2 \lambda_{4}^{V V}+4 \lambda_{5}^{V V}\right)\right)}{M_{\rho}^{2} D_{\rho}\left[(P-q)^{2}\right]}\right) \\
& v_{4}^{R R R}=-\frac{2 \sqrt{2} F_{V} k \cdot p_{-}\left(\sqrt{2} F_{V}-4(P-q)^{2} \lambda_{22}^{V}\right)\left(2 \lambda_{2}^{V A}-\lambda_{3}^{V A}\right)\left(2 \lambda_{2}^{V A}-\lambda_{4}^{V A}-2 \lambda_{5}^{V A}\right)}{F^{2} M_{\rho}^{2} D_{a_{1}}\left[\left(k+p_{-}\right)^{2}\right] D_{\rho}\left[(P-q)^{2}\right]}
\end{aligned}
$$

$$
\begin{aligned}
v_{G I 4}^{R+R R}= & \frac{2 \sqrt{2} F_{V}}{F^{2} D_{\rho}\left[(P-q)^{2}\right] D_{\rho}[s]}\left(\left(-M_{\rho}^{2}+s\right)\left(\lambda_{13}^{V}+\lambda_{14}^{V}-\lambda_{15}^{V}+\lambda_{18}^{V}+\lambda_{19}^{V}\right)+2 M_{\rho}^{2} \lambda_{21}^{V}-s \lambda_{21}^{V}+2 s \lambda_{21}^{V} \lambda_{7}^{V V}\right) \\
& +4 G_{V}\left(\sqrt{2}\left((P-q)^{2}-M_{\rho}^{2}\right) \lambda_{7}^{V}-F_{V} \lambda_{7}^{V V}+\sqrt{2} \lambda_{22}^{V}\left(-4 k \cdot p_{0}-4 k \cdot p_{-}+3 M_{\rho}^{2}-2 s+2(P-q)^{2} \lambda_{7}^{V V}\right)\right) \\
& -8\left(s\left((P-q)^{2}-M_{\rho}^{2}\right) \lambda_{7}^{V} \lambda_{21}^{V}+\lambda_{22}^{V}\left(-\left(M_{\rho}^{2}-s\right)(P-q)^{2} \lambda_{13}^{V}-\left(M_{\rho}^{2}-s\right)(P-q)^{2} \lambda_{14}^{V}+2 k \cdot p_{0} M_{\rho}^{2} \lambda_{15}^{V}\right.\right. \\
& +2 k \cdot p_{-} M_{\rho}^{2} \lambda_{15}^{V}-2\left(k \cdot p_{0}\right) s \lambda_{15}^{V}-2\left(k \cdot p_{-}\right) s \lambda_{15}^{V}+M_{\rho}^{2} s \lambda_{15}^{V}-s^{2} \lambda_{15}^{V}-2 k \cdot p_{0} M_{\rho}^{2} \lambda_{18}^{V}-2 k \cdot p_{-} M_{\rho}^{2} \lambda_{18}^{V} \\
& +2\left(k \cdot p_{0}\right) s \lambda_{18}^{V}+2\left(k \cdot p_{-}\right) s \lambda_{18}^{V}-M_{\rho}^{2} s \lambda_{18}^{V}+s^{2} \lambda_{18}^{V}-2 k \cdot p_{0} M_{\rho}^{2} \lambda_{19}^{V}-2 k \cdot p_{-} M_{\rho}^{2} \lambda_{19}^{V}+2\left(k \cdot p_{0}\right) s \lambda_{19}^{V} \\
& +2\left(k \cdot p_{-}\right) s \lambda_{19}^{V}-M_{\rho}^{2} s \lambda_{19}^{V}+s^{2} \lambda_{19}^{V}+4 k \cdot p_{0} M_{\rho}^{2} \lambda_{21}^{V}+4 k \cdot p_{-} M_{\rho}^{2} \lambda_{21}^{V}-8\left(k \cdot p_{0}\right) s \lambda_{21}^{V} \\
& \left.\left.-8\left(k \cdot p_{-}\right) s \lambda_{21}^{V}+5 M_{\rho}^{2} s \lambda_{21}^{V}-4 s^{2} \lambda_{21}^{V}+4\left(k \cdot p_{0}\right) s \lambda_{21}^{V} \lambda_{7}^{V V}+4\left(k \cdot p_{-}\right) s \lambda_{21}^{V} \lambda_{7}^{V V}+2 s^{2} \lambda_{21}^{V} \lambda_{7}^{V V}\right)\right)
\end{aligned}
$$




\section{APPENDIX D: AXIAL FORM FACTORS}

$$
\begin{aligned}
& a_{1}^{R}=\frac{\sqrt{2}}{3 F^{2}}\left(-\frac{2(P-q)^{2} F_{V}\left(\kappa_{1}^{V}-\kappa_{2}^{V}+\kappa_{3}^{V}+\kappa_{6}^{V}+\kappa_{7}^{V}-\kappa_{8}^{V}-2 \kappa_{12}^{V}-\kappa_{16}^{V}+\kappa_{17}^{V}\right)}{M_{\omega}^{2}}\right. \\
& +\frac{4\left((P-q)^{2} \kappa_{11}^{V}+s \kappa_{12}^{V}-\left(k \cdot p_{0}+k \cdot p_{-}\right) \kappa_{16}^{V}\right)\left(-G_{V}+\sqrt{2} s \lambda_{21}^{V}\right)}{D_{\rho}[s]} \\
& +\frac{1}{M_{\rho}^{2} D_{\rho}\left[\left(k+p_{-}\right)^{2}\right]}\left(F_{V}\left(2 k \cdot p_{-}+s\right)\left(2\left(k \cdot p_{-}-M_{\rho}^{2}\right) \kappa_{12}^{V}-k \cdot p_{-} \kappa_{16}^{V}\right)\right. \\
& +G_{V}\left(-4\left(2\left(k \cdot p_{-}\right)^{2}+\left(k \cdot p_{-}\right) s-M_{\rho}^{2} s\right) \kappa_{12}^{V}+2 k \cdot p_{-}\left(2 k \cdot p_{-}-2 M_{\rho}^{2}+s\right) \kappa_{16}^{V}\right) \\
& +\sqrt{2}\left(-2 \kappa_{12}^{V}\left(\left(k \cdot p_{-}-M_{\rho}^{2}\right)\left(2 k \cdot p_{0}+s\right)\left(2 k \cdot p_{-}+s\right) \lambda_{16}^{V}-2\left(k \cdot p_{-}-M_{\rho}^{2}\right)\right.\right. \\
& \times\left(2 k \cdot p_{0}+s\right)\left(2 k \cdot p_{-}+s\right) \lambda_{17}^{V}+8\left(k \cdot p_{0}\right)\left(k \cdot p_{-}\right)^{2} \lambda_{18}^{V}+8\left(k \cdot p_{-}\right)^{3} \lambda_{18}^{V} \\
& +4\left(k \cdot p_{0}\right)\left(k \cdot p_{-}\right) s \lambda_{18}^{V}+8\left(k \cdot p_{-}\right)^{2} s \lambda_{18}^{V}-4 k \cdot p_{0} M_{\rho}^{2} s \lambda_{18}^{V}-4 k \cdot p_{-} M_{\rho}^{2} s \lambda_{18}^{V} \\
& +2\left(k \cdot p_{-}\right) s^{2} \lambda_{18}^{V}-2 M_{\rho}^{2} s^{2} \lambda_{18}^{V}+8\left(k \cdot p_{0}\right)\left(k \cdot p_{-}\right)^{2} \lambda_{19}^{V}+8\left(k \cdot p_{0}\right)\left(k \cdot p_{-}\right) M_{\rho}^{2} \lambda_{19}^{V} \\
& +8\left(k \cdot p_{-}\right)^{2} M_{\rho}^{2} \lambda_{19}+4\left(k \cdot p_{0}\right)\left(k \cdot p_{-}\right) s \lambda_{19}^{V}+4\left(k \cdot p_{-}\right)^{2} s \lambda_{19}^{V}-4 k \cdot p_{0} M_{\rho}^{2} s \lambda_{19}^{V} \\
& +2\left(k \cdot p_{-}\right) s^{2} \lambda_{19}^{V}-2 M_{\rho}^{2} s^{2} \lambda_{19}^{V}-8\left(k \cdot p_{-}\right)^{3} \lambda_{21}^{V}-4\left(k \cdot p_{-}\right)^{2} s \lambda_{21}^{V}+4 k \cdot p_{-} M_{\rho}^{2} s \lambda_{21}^{V} \\
& \left.+8\left(k \cdot p_{-}\right)^{3} \lambda_{22}^{V}-8\left(k \cdot p_{-}\right)^{2} M_{\rho}^{2} \lambda_{22}^{V}+4\left(k \cdot p_{-}\right)^{2} s \lambda_{22}^{V}-4 k \cdot p_{-} M_{\rho}^{2} s \lambda_{22}^{V}\right) \\
& +k \cdot p_{-} \kappa_{16}^{V}\left(\left(2 k \cdot p_{0}+s\right)\left(2 k \cdot p_{-}+s\right) \lambda_{16}^{V}+2\left(-\left(2 k \cdot p_{0}+s\right)\left(2 k \cdot p_{-}+s\right) \lambda_{17}^{V}\right.\right. \\
& +(P-q)^{2}\left(2 k \cdot p_{-}-2 M_{\rho}^{2}+s\right) \lambda_{18}^{V}+4\left(k \cdot p_{0}\right)\left(k \cdot p_{-}\right) \lambda_{19}^{V}-8 k \cdot p_{0} M_{\rho}^{2} \lambda_{19}^{V} \\
& -4 k \cdot p_{-} M_{\rho}^{2} \lambda_{19}^{V}+2\left(k \cdot p_{0}\right) s \lambda_{19}^{V}+2\left(k \cdot p_{-}\right) s \lambda_{19}^{V}-2 M_{\rho}^{2} s \lambda_{19}^{V}+s^{2} \lambda_{19}^{V}-4\left(k \cdot p_{-}\right)^{2} \lambda_{21}^{V} \\
& \left.\left.\left.\left.+4 k \cdot p_{-} M_{\rho}^{2} \lambda_{21}^{V}-2\left(k \cdot p_{-}\right) s \lambda_{21}^{V}+4\left(k \cdot p_{-}\right)^{2} \lambda_{22}^{V}+2\left(k \cdot p_{-}\right) s \lambda_{22}^{V}\right)\right)\right)\right) \\
& +\frac{1}{M_{\rho}^{2} D_{\rho}\left[\left(k+p_{0}\right)^{2}\right]}\left(F_{V}\left(4 k \cdot p_{0}+2 k \cdot p_{-}+s\right)\left(2\left(k \cdot p_{0}-M_{\rho}^{2}\right) \kappa_{12}^{V}-k \cdot p_{0} \kappa_{16}^{V}\right)\right. \\
& +G_{V}\left(-4\left(4\left(k \cdot p_{0}\right)^{2}-M_{\rho}^{2}\left(2 k \cdot p_{-}+s\right)+k \cdot p_{0}\left(2 k \cdot p_{-}-2 M_{\rho}^{2}+s\right)\right) \kappa_{12}^{V}+2 k \cdot p_{0}\right. \\
& \left.\times\left(4 k \cdot p_{0}+2 k \cdot p_{-}-2 M_{\rho}^{2}+s\right) \kappa_{16}^{V}\right)+\sqrt{2}\left(k \cdot p _ { 0 } \kappa _ { 1 6 } ^ { V } \left(\left(2 k \cdot p_{-}+s\right)\left(4 k \cdot p_{0}+2 k \cdot p_{-}+s\right) \lambda_{16}^{V}\right.\right. \\
& +2\left(-\left(2 k \cdot p_{-}+s\right)\left(4 k \cdot p_{0}+2 k \cdot p_{-}+s\right) \lambda_{17}^{V}+\left(8\left(k \cdot p_{0}\right)^{2}+12\left(k \cdot p_{0}\right)\left(k \cdot p_{-}\right)\right.\right. \\
& \left.+4\left(k \cdot p_{-}\right)^{2}-4 k \cdot p_{0} M_{\rho}^{2}-4 k \cdot p_{-} M_{\rho}^{2}+6\left(k \cdot p_{0}\right) s+4\left(k \cdot p_{-}\right) s-2 M_{\rho}^{2} s+s^{2}\right) \lambda_{18}^{V} \\
& +8\left(k \cdot p_{0}\right)\left(k \cdot p_{-}\right) \lambda_{19}^{V}+4\left(k \cdot p_{-}\right)^{2} \lambda_{19}^{V}-4 k \cdot p_{-} M_{\rho}^{2} \lambda_{19}^{V}+4\left(k \cdot p_{0}\right) s \lambda_{19}^{V} \\
& +4\left(k \cdot p_{-}\right) s \lambda_{19}^{V}-2 M_{\rho}^{2} s \lambda_{19}^{V}+s^{2} \lambda_{19}^{V}-8\left(k \cdot p_{0}\right)^{2} \lambda_{21}^{V}-4\left(k \cdot p_{0}\right)\left(k \cdot p_{-}\right) \lambda_{21}^{V} \\
& \left.\left.+4 k \cdot p_{0} M_{\rho}^{2} \lambda_{21}^{V}-2\left(k \cdot p_{0}\right) s \lambda_{21}^{V}+8\left(k \cdot p_{0}\right)^{2} \lambda_{22}^{V}+4\left(k \cdot p_{0}\right)\left(k \cdot p_{-}\right) \lambda_{22}^{V}+2\left(k \cdot p_{0}\right) s \lambda_{22}^{V}\right)\right) \\
& -2 \kappa_{12}^{V}\left(\left(k \cdot p_{0}-M_{\rho}^{2}\right)\left(2 k \cdot p_{-}+s\right)\left(4 k \cdot p_{0}+2 k \cdot p_{-}+s\right) \lambda_{16}^{V}+2\left(-\left(k \cdot p_{0}-M_{\rho}^{2}\right)\right.\right. \\
& \times\left(2 k \cdot p_{-}+s\right)\left(4 k \cdot p_{0}+2 k \cdot p_{-}+s\right) \lambda_{17}^{V}+(P-q)^{2}\left(4\left(k \cdot p_{0}\right)^{2}-M_{\rho}^{2}\left(2 k \cdot p_{-}+s\right)\right. \\
& \left.+k \cdot p_{0}\left(2 k \cdot p_{-}-2 M_{\rho}^{2}+s\right)\right) \lambda_{18}^{V}+8\left(k \cdot p_{0}\right)^{2}\left(k \cdot p_{-}\right) \lambda_{19}^{V}+4\left(k \cdot p_{0}\right)\left(k \cdot p_{-}\right)^{2} \lambda_{19}^{V} \\
& -4\left(k \cdot p_{0}\right)\left(k \cdot p_{-}\right) M_{\rho}^{2} \lambda_{19}^{V}-4\left(k \cdot p_{-}\right)^{2} M_{\rho}^{2} \lambda_{19}^{V}+4\left(k \cdot p_{0}\right)^{2} s \lambda_{19}^{V}+4\left(k \cdot p_{0}\right)\left(k \cdot p_{-}\right) s \lambda_{19}^{V} \\
& -2 k \cdot p_{0} M_{\rho}^{2} s \lambda_{19}^{V}-4 k \cdot p_{-} M_{\rho}^{2} s \lambda_{19}^{V}+\left(k \cdot p_{0}\right) s^{2} \lambda_{19}^{V}-M_{\rho}^{2} s^{2} \lambda_{19}^{V}-8\left(k \cdot p_{0}\right)^{3} \lambda_{21}^{V} \\
& -4\left(k \cdot p_{0}\right)^{2}\left(k \cdot p_{-}\right) \lambda_{21}^{V}+4\left(k \cdot p_{0}\right)^{2} M_{\rho}^{2} \lambda_{21}^{V}+4\left(k \cdot p_{0}\right)\left(k \cdot p_{-}\right) M_{\rho}^{2} \lambda_{21}^{V}-2\left(k \cdot p_{0}\right)^{2} s \lambda_{21}^{V} \\
& +2 k \cdot p_{0} M_{\rho}^{2} s \lambda_{21}^{V}+8\left(k \cdot p_{0}\right)^{3} \lambda_{22}^{V}+4\left(k \cdot p_{0}\right)^{2}\left(k \cdot p_{-}\right) \lambda_{22}^{V}-8\left(k \cdot p_{0}\right)^{2} M_{\rho}^{2} \lambda_{22}^{V} \\
& \left.\left.\left.\left.-4\left(k \cdot p_{0}\right)\left(k \cdot p_{-}\right) M_{\rho}^{2} \lambda_{22}^{V}+2\left(k \cdot p_{0}\right)^{2} s \lambda_{22}^{V}-2 k \cdot p_{0} M_{\rho}^{2} s \lambda_{22}^{V}\right)\right)\right)\right) \\
& \left.+\frac{2(P-q)^{2}\left(\kappa_{5}^{A}-\kappa_{6}^{A}+\kappa_{7}^{A}\right)\left(-F_{A}+2 \sqrt{2}(P-q)^{2} \lambda_{17}^{A}\right)}{D_{a_{1}}\left[(P-q)^{2}\right]}\right)
\end{aligned}
$$




$$
\begin{aligned}
& a_{1}^{R R}=\frac{1}{3 \sqrt{2} F^{2}}\left(\frac{4 s F_{V} \kappa_{3}^{V V}\left(-\sqrt{2} G_{V}+2 s \lambda_{21}^{V}\right)}{M_{\omega}^{2} D_{\rho}[s]}\right. \\
& -\frac{1}{M_{\rho}^{2} M_{\omega}^{2} D_{\rho}\left[\left(k+p_{-}\right)^{2}\right]}\left(F _ { V } \left(\sqrt{2}\left(2 k \cdot p_{-}+s\right) F_{V}\left(M_{\rho}^{2} \kappa_{3}^{V V}+D_{\rho}\left[\left(k+p_{-}\right)^{2}\right] \kappa_{4}^{V V}\right)\right.\right. \\
& +2 \sqrt{2} G_{V}\left(M_{\rho}^{2}\left(2 k \cdot p_{-}-s\right) \kappa_{3}^{V V}-\left(2 k \cdot p_{-}+s\right) D_{\rho}\left[\left(k+p_{-}\right)^{2}\right] \kappa_{4}^{V V}\right) \\
& +2\left(2 k \cdot p_{-}-M_{\rho}^{2}\right)\left(2 k \cdot p_{-}+s\right) \kappa_{4}^{V V}\left(\left(2 k \cdot p_{0}+s\right) \lambda_{16}^{V}-2\left(2 k \cdot p_{0}+s\right) \lambda_{17}^{V}+4 k \cdot p_{0} \lambda_{18}^{V}\right. \\
& \left.+4 k \cdot p_{-} \lambda_{18}^{V}+2 s \lambda_{18}^{V}+4 k \cdot p_{0} \lambda_{19}^{V}+2 s \lambda_{19}^{V}-4 k \cdot p_{-} \lambda_{21}^{V}+4 k \cdot p_{-} \lambda_{22}^{V}\right)-2 M_{\rho}^{2} \kappa_{3}^{V V} \\
& \times\left(\left(2 k \cdot p_{0}+s\right)\left(2 k \cdot p_{-}+s\right) \lambda_{16}^{V}-2\left(2 k \cdot p_{0}+s\right)\left(2 k \cdot p_{-}+s\right) \lambda_{17}^{V}-2\left(2 k \cdot p_{-}-s\right)\right. \\
& \times(P-q)^{2} \lambda_{18}^{V}-24\left(k \cdot p_{0}\right)\left(k \cdot p_{-}\right) \lambda_{19}^{V}-16\left(k \cdot p_{-}\right)^{2} \lambda_{19}^{V}+4\left(k \cdot p_{0}\right) s \lambda_{19}^{V}-4\left(k \cdot p_{-}\right) s \lambda_{19}^{V} \\
& \left.\left.\left.+2 s^{2} \lambda_{19}^{V}+8\left(k \cdot p_{-}\right)^{2} \lambda_{21}^{V}-4\left(k \cdot p_{-}\right) s \lambda_{21}^{V}+8\left(k \cdot p_{-}\right)^{2} \lambda_{22}^{V}+4\left(k \cdot p_{-}\right) s \lambda_{22}^{V}\right)\right)\right) \\
& +\frac{1}{M_{\rho}^{2} M_{\omega}^{2} D_{\rho}\left[\left(k+p_{0}\right)^{2}\right]}\left(F _ { V } \left(-\sqrt{2} F_{V}\left(4 k \cdot p_{0}+2 k \cdot p_{-}+s\right)\left(M_{\rho}^{2} \kappa_{3}^{V V}+D_{\rho}\left[\left(k+p_{0}\right)^{2}\right] \kappa_{4}^{V V}\right)\right.\right. \\
& +2 \sqrt{2} G_{V}\left(M_{\rho}^{2}\left(2 k \cdot p_{-}+s\right) \kappa_{3}^{V V}+\left(4 k \cdot p_{0}+2 k \cdot p_{-}+s\right) D_{\rho}\left[\left(k+p_{0}\right)^{2}\right] \kappa_{4}^{V V}\right) \\
& -2\left(2 k \cdot p_{0}-M_{\rho}^{2}\right)\left(4 k \cdot p_{0}+2 k \cdot p_{-}+s\right) \kappa_{4}^{V V}\left(\left(2 k \cdot p_{-}+s\right) \lambda_{16}^{V}-2\left(2 k \cdot p_{-}+s\right) \lambda_{17}^{V}\right. \\
& \left.+4 k \cdot p_{0} \lambda_{18}^{V}+4 k \cdot p_{-} \lambda_{18}^{V}+2 s \lambda_{18}^{V}+4 k \cdot p_{-} \lambda_{19}^{V}+2 s \lambda_{19}^{V}-4 k \cdot p_{0} \lambda_{21}^{V}+4 k \cdot p_{0} \lambda_{22}^{V}\right) \\
& +2 M_{\rho}^{2} \kappa_{3}^{V V}\left(\left(2 k \cdot p_{-}+s\right)\left(4 k \cdot p_{0}+2 k \cdot p_{-}+s\right) \lambda_{16}^{V}+2\left(-\left(2 k \cdot p_{-}+s\right)\right.\right. \\
& \times\left(4 k \cdot p_{0}+2 k \cdot p_{-}+s\right) \lambda_{17}^{V}+\left(2 k \cdot p_{-}+s\right)(P-q)^{2} \lambda_{18}^{V}+4\left(k \cdot p_{-}\right)^{2} \lambda_{19}^{V} \\
& +4\left(k \cdot p_{-}\right) s \lambda_{19}^{V}+s^{2} \lambda_{19}^{V}-4\left(k \cdot p_{0}\right)\left(k \cdot p_{-}\right) \lambda_{21}^{V}-2\left(k \cdot p_{0}\right) s \lambda_{21}^{V}+8\left(k \cdot p_{0}\right)^{2} \lambda_{22}^{V} \\
& \left.\left.\left.\left.+4\left(k \cdot p_{0}\right)\left(k \cdot p_{-}\right) \lambda_{22}^{V}+2\left(k \cdot p_{0}\right) s \lambda_{22}^{V}\right)\right)\right)\right) \\
& +\frac{2(P-q)^{2} F_{V}\left(\kappa_{2}^{V A}-\kappa_{3}^{V A}-\kappa_{4}^{V A}\right)\left(-\sqrt{2} F_{A}+4(P-q)^{2} \lambda_{17}^{A}\right)}{M_{\omega}^{2} D_{a_{1}}\left[(P-q)^{2}\right]} \\
& -\frac{4(P-q)^{2} \kappa_{5}^{V A}\left(-\sqrt{2} G_{V}+2 s \lambda_{21}^{V}\right)\left(-F_{A}+2 \sqrt{2}(P-q)^{2} \lambda_{17}^{A}\right)}{D_{a_{1}}\left[(P-q)^{2}\right] D_{\rho}[s]} \\
& -\frac{2\left(F_{A}-2 \sqrt{2}(P-q)^{2} \lambda_{17}^{A}\right)}{M_{\rho}^{2} D_{a_{1}}\left[(P-q)^{2}\right] D_{\rho}\left[\left(k+p_{-}\right)^{2}\right]}\left(k \cdot p _ { - } \kappa _ { 1 6 } ^ { V } \left(-2\left(-\left(2 M_{\rho}^{2}-s\right)\left(2 k \cdot p_{-}+s\right)\right.\right.\right. \\
& \left.+2 k \cdot p_{0}\left(2 k \cdot p_{-}-4 M_{\rho}^{2}+s\right)\right) \lambda_{2}^{V A}-2 M_{\rho}^{2}\left(4 k \cdot p_{0}+2 k \cdot p_{-}+s\right) \lambda_{3}^{V A} \\
& \left.+\left(2 k \cdot p_{-}+s\right)\left(\left(2 k \cdot p_{0}+4 k \cdot p_{-}-2 M_{\rho}^{2}+s\right) \lambda_{4}^{V A}+2\left(2 k \cdot p_{0}+s\right) \lambda_{5}^{V A}\right)\right) \\
& +2 \kappa_{12}^{V}\left(2 \left(\left(k \cdot p_{-}\right) s^{2}-M_{\rho}^{2} s^{2}+2\left(k \cdot p_{-}\right)^{2}\left(2 M_{\rho}^{2}+s\right)+2 k \cdot p_{0}\left(2\left(k \cdot p_{-}\right)^{2}\right.\right.\right. \\
& \left.\left.-M_{\rho}^{2} s+k \cdot p_{-}\left(2 M_{\rho}^{2}+s\right)\right)\right) \lambda_{2}^{V A}-2 k \cdot p_{-} M_{\rho}^{2}\left(4 k \cdot p_{0}+2 k \cdot p_{-}+s\right) \lambda_{3}^{V A} \\
& -\left(2 k \cdot p_{-}+s\right)\left(\left(4\left(k \cdot p_{-}\right)^{2}-2 k \cdot p_{-} M_{\rho}^{2}+2 k \cdot p_{0}\left(k \cdot p_{-}-M_{\rho}^{2}\right)+\left(k \cdot p_{-}\right) s\right.\right. \\
& \left.\left.\left.\left.-M_{\rho}^{2} s\right) \lambda_{4}^{V A}+2\left(k \cdot p_{-}-M_{\rho}^{2}\right)\left(2 k \cdot p_{0}+s\right) \lambda_{5}^{V A}\right)\right)\right) \\
& +\frac{2\left(F_{A}-2 \sqrt{2}(P-q)^{2} \lambda_{17}^{A}\right)}{M_{\rho}^{2} D_{a_{1}}\left[(P-q)^{2}\right] D_{\rho}\left[\left(k+p_{0}\right)^{2}\right]}\left(-k \cdot p_{0} \kappa_{16}^{V}\left(-2\left(2 k \cdot p_{-}+s\right)\left(4 k \cdot p_{0}+2 k \cdot p_{-}\right.\right.\right. \\
& \left.-2 M_{\rho}^{2}+s\right) \lambda_{2}^{V A}-2 M_{\rho}^{2}\left(2 k \cdot p_{-}+s\right) \lambda_{3}^{V A}+\left(4 k \cdot p_{0}+2 k \cdot p_{-}+s\right)\left(\left(4 k \cdot p_{0}+2 k \cdot p_{-}\right.\right. \\
& \left.\left.\left.-2 M_{\rho}^{2}+s\right) \lambda_{4}^{V A}+2\left(2 k \cdot p_{-}+s\right) \lambda_{5}^{V A}\right)\right)+2 \kappa_{12}^{V}\left(-2\left(2 k \cdot p_{-}+s\right)\left(4\left(k \cdot p_{0}\right)^{2}\right.\right. \\
& \left.-M_{\rho}^{2}\left(2 k \cdot p_{-}+s\right)+k \cdot p_{0}\left(2 k \cdot p_{-}-2 M_{\rho}^{2}+s\right)\right) \lambda_{2}^{V A}+2 k \cdot p_{0} M_{\rho}^{2}\left(2 k \cdot p_{-}+s\right) \lambda_{3}^{V A} \\
& +\left(4 k \cdot p_{0}+2 k \cdot p_{-}+s\right)\left(\left(4\left(k \cdot p_{0}\right)^{2}-M_{\rho}^{2}\left(2 k \cdot p_{-}+s\right)+k \cdot p_{0}\left(2 k \cdot p_{-}-2 M_{\rho}^{2}+s\right)\right) \lambda_{4}^{V A}\right. \\
& \left.\left.\left.\left.+2\left(k \cdot p_{0}-M_{\rho}^{2}\right)\left(2 k \cdot p_{-}+s\right) \lambda_{5}^{V A}\right)\right)\right)\right)
\end{aligned}
$$




$$
\begin{aligned}
a_{1}^{R R R}= & -\frac{F_{V}\left(\sqrt{2} F_{A}-4(P-q)^{2} \lambda_{17}^{A}\right)}{3 \sqrt{2} F^{2} M_{\rho}^{2} M_{\omega}^{2} D_{a_{1}}\left[(P-q)^{2}\right] D_{\rho}\left[\left(k+p_{0}\right)^{2}\right] D_{\rho}\left[\left(k+p_{-}\right)^{2}\right]}\left(-M_{\rho}^{2}\left(2 k \cdot p_{-}+s\right)\right. \\
& \times D_{\rho}\left[\left(k+p_{-}\right)^{2}\right] \kappa_{3}^{V V}\left(2\left(2 k \cdot p_{-}+s\right) \lambda_{2}^{V A}+4 k \cdot p_{0} \lambda_{3}^{V A}-\left(4 k \cdot p_{0}+2 k \cdot p_{-}+s\right)\right. \\
& \left.\times\left(\lambda_{4}^{V A}+2 \lambda_{5}^{V A}\right)\right)+D_{\rho}\left[\left(k+p_{0}\right)^{2}\right]\left(2 D _ { \rho } [ ( k + p _ { - } ) ^ { 2 } ] \kappa _ { 4 } ^ { V V } \left(-2\left(3 k \cdot p_{0}+k \cdot p_{-}+s\right)\right.\right. \\
& \times\left(2 k \cdot p_{-}+s\right) \lambda_{2}^{V A}+\left(8\left(k \cdot p_{0}\right)^{2}+6\left(k \cdot p_{-}\right)^{2}+5\left(k \cdot p_{-}\right) s+s^{2}+5 k \cdot p_{0}\right. \\
& \left.\left.\times\left(2 k \cdot p_{-}+s\right)\right) \lambda_{4}^{V A}+2\left(3 k \cdot p_{0}+k \cdot p_{-}+s\right)\left(2 k \cdot p_{-}+s\right) \lambda_{5}^{V A}\right)+M_{\rho}^{2} \kappa_{3}^{V V} \\
& \times\left(2\left(12\left(k \cdot p_{0}\right)\left(k \cdot p_{-}\right)+8\left(k \cdot p_{-}\right)^{2}-2\left(k \cdot p_{0}\right) s+2\left(k \cdot p_{-}\right) s-s^{2}\right) \lambda_{2}^{V A}\right. \\
& \left.\left.\left.-4 k \cdot p_{-}\left(4 k \cdot p_{0}+2 k \cdot p_{-}+s\right) \lambda_{3}^{V A}+\left(2 k \cdot p_{0}+s\right)\left(2 k \cdot p_{-}+s\right)\left(\lambda_{4}^{V A}+2 \lambda_{5}^{V A}\right)\right)\right)\right)
\end{aligned}
$$

$$
\begin{aligned}
& a_{2}^{R}=\frac{4 \sqrt{2}}{3 F^{2}}\left(-\frac{2(P-q)^{2} F_{V}\left(\kappa_{1}^{V}-\kappa_{2}^{V}+\kappa_{3}^{V}\right)}{M_{\omega}^{2} D_{\pi}\left[(P-q)^{2}\right]}\right. \\
& +\frac{F_{V}\left(3 \kappa_{1}^{V}-3 \kappa_{2}^{V}+3 \kappa_{3}^{V}+\kappa_{6}^{V}+\kappa_{7}^{V}-\kappa_{8}^{V}-2 \kappa_{12}^{V}-\kappa_{16}^{V}+\kappa_{17}^{V}\right)}{M_{\omega}^{2}} \\
& +\frac{2 \sqrt{2} k \cdot p_{0}\left(2 \kappa_{12}^{V}+\kappa_{16}^{V}\right)\left(\lambda_{18}^{V}+2 \lambda_{19}^{V}\right)}{D_{\rho}\left[\left(k+p_{0}\right)^{2}\right]}-\frac{2 k \cdot p_{0}\left(2 \kappa_{12}^{V}+\kappa_{16}^{V}\right)\left(-G_{V}+2 \sqrt{2} k \cdot p_{0} \lambda_{21}^{V}\right)}{D_{\pi}\left[(P-q)^{2}\right] D_{\rho}\left[\left(k+p_{0}\right)^{2}\right]} \\
& -\frac{2 k \cdot p_{-}\left(2 \kappa_{12}^{V}+\kappa_{16}^{V}\right)\left(-G_{V}+2 \sqrt{2} k \cdot p_{-} \lambda_{21}^{V}\right)}{D_{\pi}\left[(P-q)^{2}\right] D_{\rho}\left[\left(k+p_{-}\right)^{2}\right]}+\frac{2\left(\kappa_{11}^{V}+\kappa_{12}^{V}\right)\left(G_{V}-\sqrt{2} s \lambda_{21}^{V}\right)}{D_{\rho}[s]} \\
& -\frac{2\left(k \cdot p_{0}+k \cdot p_{-}\right)\left(2 \kappa_{12}^{V}+\kappa_{16}^{V}\right)\left(G_{V}-\sqrt{2} s \lambda_{21}^{V}\right)}{D_{\pi}\left[(P-q)^{2}\right] D_{\rho}[s]}+\frac{1}{M_{\rho}^{2} D_{\rho}\left[\left(k+p_{-}\right)^{2}\right]} \\
& \times\left(G_{V}\left(4\left(k \cdot p_{-}-M_{\rho}^{2}\right) \kappa_{12}^{V}-2 k \cdot p_{-} \kappa_{16}^{V}\right)+F_{V}\left(-2\left(k \cdot p_{-}-M_{\rho}^{2}\right) \kappa_{12}^{V}+k \cdot p_{-} \kappa_{16}^{V}\right)\right. \\
& +\sqrt{2}\left(-k \cdot p_{-} \kappa_{16}^{V}\left(\left(2 k \cdot p_{0}+s\right) \lambda_{16}^{V}-2\left(2 k \cdot p_{0}+s\right) \lambda_{17}^{V}+4 k \cdot p_{0} \lambda_{18}^{V}+4 k \cdot p_{-} \lambda_{18}^{V}\right.\right. \\
& \left.-2 M_{\rho}^{2} \lambda_{18}^{V}+2 s \lambda_{18}^{V}+4 k \cdot p_{0} \lambda_{19}^{V}+2 s \lambda_{19}^{V}-4 k \cdot p_{-} \lambda_{21}^{V}+4 k \cdot p_{-} \lambda_{22}^{V}\right)+2 \kappa_{12}^{V} \\
& \times\left(\left(k \cdot p_{-}-M_{\rho}^{2}\right)\left(2 k \cdot p_{0}+s\right) \lambda_{16}^{V}-2\left(k \cdot p_{-}-M_{\rho}^{2}\right)\left(2 k \cdot p_{0}+s\right) \lambda_{17}^{V}+2\left(2\left(k \cdot p_{-}\right)^{2}\right.\right. \\
& \left.+2 k \cdot p_{0}\left(k \cdot p_{-}-M_{\rho}^{2}\right)-M_{\rho}^{2} s+k \cdot p_{-}\left(-M_{\rho}^{2}+s\right)\right) \lambda_{18}^{V}+2\left(k \cdot p_{-}-M_{\rho}^{2}\right) \\
& \left.\left.\left.\times\left(\left(2 k \cdot p_{0}+s\right) \lambda_{19}^{V}+2 k \cdot p_{-}\left(-\lambda_{21}^{V}+\lambda_{22}^{V}\right)\right)\right)\right)\right) \\
& \left.+\frac{\left(\kappa_{5}^{A}-\kappa_{6}^{A}+\kappa_{7}^{A}\right)\left(F_{A}-2 \sqrt{2}(P-q)^{2} \lambda_{17}^{A}\right)}{D_{a_{1}}\left[(P-q)^{2}\right]}\right)
\end{aligned}
$$




$$
\begin{aligned}
a_{2}^{R R}= & \frac{2 \sqrt{2}}{3 F^{2}}\left(\frac{8 k \cdot p_{0} F_{V} \kappa_{3}^{V V}\left(\lambda_{18}^{V}+2 \lambda_{19}^{V}\right)}{M_{\omega}^{2} D_{\rho}\left[\left(k+p_{0}\right)^{2}\right]}+\frac{4 k \cdot p_{0} F_{V} \kappa_{3}^{V V}\left(\sqrt{2} G_{V}-4 k \cdot p_{0} \lambda_{21}^{V}\right)}{M_{\omega}^{2} D_{\pi}\left[(P-q)^{2}\right] D_{\rho}\left[\left(k+p_{0}\right)^{2}\right]}\right. \\
& +\frac{4 k \cdot p_{-} F_{V} \kappa_{3}^{V V}\left(\sqrt{2} G_{V}-4 k \cdot p_{-} \lambda_{21}^{V}\right)}{M_{\omega}^{2} D_{\pi}\left[(P-q)^{2}\right] D_{\rho}\left[\left(k+p_{-}\right)^{2}\right]}+\frac{2 F_{V} \kappa_{3}^{V V}\left(\sqrt{2} G_{V}-2 s \lambda_{21}^{V}\right)}{M_{\omega}^{2} D_{\rho}[s]} \\
& +\frac{F_{V}}{M_{\rho}^{2} M_{\omega}^{2} D_{\rho}\left[\left(k+p_{-}\right)^{2}\right]}\left(\sqrt{2} F_{V}\left(M_{\rho}^{2} \kappa_{3}^{V V}+D_{\rho}\left[\left(k+p_{-}\right)^{2}\right] \kappa_{4}^{V V}\right)\right. \\
& -2 \sqrt{2} G_{V}\left(M_{\rho}^{2} \kappa_{3}^{V V}+D_{\rho}\left[\left(k+p_{-}\right)^{2}\right] \kappa_{4}^{V V}\right)-2 M_{\rho}^{2} \kappa_{3}^{V V}\left(\left(2 k \cdot p_{0}+s\right) \lambda_{16}^{V}\right. \\
& -2\left(2 k \cdot p_{0}+s\right) \lambda_{17}^{V}+4 k \cdot p_{0} \lambda_{18}^{V}+2 s \lambda_{18}^{V}+4 k \cdot p_{0} \lambda_{19}^{V}+2 s \lambda_{19}^{V}-4 k \cdot p_{-} \lambda_{21}^{V} \\
& \left.+4 k \cdot p_{-} \lambda_{22}^{V}\right)+2\left(2 k \cdot p_{-}-M_{\rho}^{2}\right) \kappa_{4}^{V V}\left(\left(2 k \cdot p_{0}+s\right) \lambda_{16}^{V}-2\left(2 k \cdot p_{0}+s\right) \lambda_{17}^{V}\right. \\
& +4 k \cdot p_{0} \lambda_{18}^{V}+4 k \cdot p_{-} \lambda_{18}^{V}+2 s \lambda_{18}^{V}+4 k \cdot p_{0} \lambda_{19}^{V}+2 s \lambda_{19}^{V}-4 k \cdot p_{-} \lambda_{21}^{V} \\
& \left.\left.+4 k \cdot p_{-} \lambda_{22}^{V}\right)\right)+\frac{F_{V}\left(\kappa_{2}^{V A}-\kappa_{3}^{V A}-\kappa_{4}^{V A}\right)\left(\sqrt{2} F_{A}-4(P-q)^{2} \lambda_{17}^{A}\right)}{M_{\omega}^{2} D_{a_{1}}\left[(P-q)^{2}\right]} \\
& +\frac{2 \kappa_{5}^{V A}\left(\sqrt{2} G_{V}-2 s \lambda_{21}^{V}\right)\left(F_{A}-2 \sqrt{2}(P-q)^{2} \lambda_{17}^{A}\right)}{D_{a_{1}}\left[(P-q)^{2}\right] D_{\rho}[s]} \\
& +\frac{4 k \cdot p_{0}\left(2 \kappa_{12}^{V}+\kappa_{16}^{V}\right)\left(F_{A}-2 \sqrt{2}(P-q)^{2} \lambda_{17}^{A}\right)\left(2 \lambda_{2}^{V A}-\lambda_{3}^{V A}\right)}{D_{a_{1}}\left[(P-q)^{2}\right] D_{\rho}\left[\left(k+p_{0}\right)^{2}\right]} \\
& +\frac{2\left(F_{A}-2 \sqrt{2}(P-q)^{2} \lambda_{17}^{A}\right)}{M_{\rho}^{2} D_{a_{1}}\left[(P-q)^{2}\right] D_{\rho}\left[\left(k+p_{-}\right)^{2}\right]}\left(k \cdot p _ { - } \kappa _ { 1 6 } ^ { V } \left(-2\left(2 k \cdot p_{0}+s\right) \lambda_{2}^{V A}\right.\right. \\
& \left.+\left(2 k \cdot p_{0}+4 k \cdot p_{-}-2 M_{\rho}^{2}+s\right) \lambda_{4}^{V A}+2\left(2 k \cdot p_{0}+s\right) \lambda_{5}^{V A}\right)+2 \kappa_{12}^{V} \\
& \times\left(2\left(k \cdot p_{-}-M_{\rho}^{2}\right)\left(2 k \cdot p_{0}+s\right) \lambda_{2}^{V A}+\left(-2\left(k \cdot p_{0}\right)\left(k \cdot p_{-}\right)-4\left(k \cdot p_{-}\right)^{2}\right.\right. \\
& \left.\left.\left.\left.+2 k \cdot p_{0} M_{\rho}^{2}+2 k \cdot p_{-} M_{\rho}^{2}-\left(k \cdot p_{-}\right) s+M_{\rho}^{2} s\right) \lambda_{4}^{V A}-2\left(k \cdot p_{-}-M_{\rho}^{2}\right)\left(2 k \cdot p_{0}+s\right) \lambda_{5}^{V A}\right)\right)\right)
\end{aligned}
$$

$$
\begin{aligned}
a_{2}^{R R R}= & -\frac{2 \sqrt{2} F_{V}\left(\sqrt{2} F_{A}-4(P-q)^{2} \lambda_{17}^{A}\right)}{3 F^{2} M_{\rho}^{2} M_{\omega}^{2} D_{a_{1}}\left[(P-q)^{2}\right] D_{\rho}\left[\left(k+p_{0}\right)^{2}\right] D_{\rho}\left[\left(k+p_{-}\right)^{2}\right]} \\
& \times\left(4 k \cdot p_{0} M_{\rho}^{2} D_{\rho}\left[\left(k+p_{-}\right)^{2}\right] \kappa_{3}^{V V}\left(-2 \lambda_{2}^{V A}+\lambda_{3}^{V A}\right)+D_{\rho}\left[\left(k+p_{0}\right)^{2}\right]\left(M_{\rho}^{2}\left(2 k \cdot p_{0}+s\right) \kappa_{3}^{V V}\right.\right. \\
& \times\left(2 \lambda_{2}^{V A}-\lambda_{4}^{V A}-2 \lambda_{5}^{V A}\right)+D_{\rho}\left[\left(k+p_{-}\right)^{2}\right] \kappa_{4}^{V V}\left(2\left(2 k \cdot p_{0}+s\right) \lambda_{2}^{V A}\right. \\
& \left.\left.\left.-\left(2 k \cdot p_{0}+4 k \cdot p_{-}+s\right) \lambda_{4}^{V A}-2\left(2 k \cdot p_{0}+s\right) \lambda_{5}^{V A}\right)\right)\right)
\end{aligned}
$$




$$
\begin{aligned}
& a_{3}^{R}=\frac{\sqrt{2}}{3 F^{2}}\left(-\frac{4 F_{V}\left(k \cdot p_{0}-k \cdot p_{-}\right)\left(\kappa_{1}^{V}-\kappa_{2}^{V}+\kappa_{3}^{V}+\kappa_{6}^{V}+\kappa_{7}^{V}-\kappa_{8}^{V}-\kappa_{17}^{V}\right)}{M_{\omega}^{2}}\right. \\
& -\frac{4\left(k \cdot p_{0}-k \cdot p_{-}\right)\left(2 \kappa_{11}^{V}-\kappa_{16}^{V}\right)\left(G_{V}-\sqrt{2} s \lambda_{21}^{V}\right)}{D_{\rho}[s]} \\
& +\frac{1}{M_{\rho}^{2} D_{\rho}\left[\left(k+p_{0}\right)^{2}\right]}\left(-2 G_{V}\left(2 \left(4\left(k \cdot p_{0}\right)^{2}+M_{\rho}^{2}\left(2 k \cdot p_{-}+s\right)-k \cdot p_{0}\right.\right.\right. \\
& \left.\left.\times\left(2 k \cdot p_{-}+2 M_{\rho}^{2}+s\right)\right) \kappa_{12}^{V}+k \cdot p_{0}\left(-4 k \cdot p_{0}+2 k \cdot p_{-}+2 M_{\rho}^{2}+s\right) \kappa_{16}^{V}\right) \\
& +F_{V}\left(2\left(4\left(k \cdot p_{0}\right)^{2}-k \cdot p_{0}\left(2 k \cdot p_{-}+s\right)+M_{\rho}^{2}\left(2 k \cdot p_{-}+s\right)\right) \kappa_{12}^{V}+k \cdot p_{0}\right. \\
& \left.\times\left(-4 k \cdot p_{0}+2 k \cdot p_{-}+4 M_{\rho}^{2}+s\right) \kappa_{16}^{V}\right)-\sqrt{2}\left(k \cdot p _ { 0 } \kappa _ { 1 6 } ^ { V } \left(-\left(4 k \cdot p_{0}-2 k \cdot p_{-}\right.\right.\right. \\
& \left.-4 M_{\rho}^{2}-s\right)\left(2 k \cdot p_{-}+s\right) \lambda_{16}^{V}+2\left(\left(4 k \cdot p_{0}-2 k \cdot p_{-}-4 M_{\rho}^{2}-s\right)\left(2 k \cdot p_{-}+s\right) \lambda_{17}^{V}\right. \\
& +\left(-8\left(k \cdot p_{0}\right)^{2}+4\left(k \cdot p_{-}\right)^{2}-2 k \cdot p_{0}\left(2 k \cdot p_{-}-2 M_{\rho}^{2}+s\right)+4 k \cdot p_{-}\left(M_{\rho}^{2}+s\right)\right. \\
& \left.+s\left(2 M_{\rho}^{2}+s\right)\right) \lambda_{18}^{V}-8\left(k \cdot p_{0}\right)\left(k \cdot p_{-}\right) \lambda_{19}^{V}+4\left(k \cdot p_{-}\right)^{2} \lambda_{19}^{V}+4 k \cdot p_{-} M_{\rho}^{2} \lambda_{19}^{V} \\
& -4\left(k \cdot p_{0}\right) s \lambda_{19}^{V}+4\left(k \cdot p_{-}\right) s \lambda_{19}^{V}+2 M_{\rho}^{2} s \lambda_{19}^{V}+s^{2} \lambda_{19}^{V}+8\left(k \cdot p_{0}\right)^{2} \lambda_{21}^{V} \\
& -4\left(k \cdot p_{0}\right)\left(k \cdot p_{-}\right) \lambda_{21}^{V}-4 k \cdot p_{0} M_{\rho}^{2} \lambda_{21}^{V}-2\left(k \cdot p_{0}\right) s \lambda_{21}^{V}-8\left(k \cdot p_{0}\right)^{2} \lambda_{22}^{V} \\
& \left.\left.+4\left(k \cdot p_{0}\right)\left(k \cdot p_{-}\right) \lambda_{22}^{V}+8 k \cdot p_{0} M_{\rho}^{2} \lambda_{22}^{V}+2\left(k \cdot p_{0}\right) s \lambda_{22}^{V}\right)\right)+2 \kappa_{12}^{V}\left(\left(2 k \cdot p_{-}+s\right)\right. \\
& \times\left(4\left(k \cdot p_{0}\right)^{2}-k \cdot p_{0}\left(2 k \cdot p_{-}+s\right)+M_{\rho}^{2}\left(2 k \cdot p_{-}+s\right)\right) \lambda_{16}^{V}+2\left(-\left(2 k \cdot p_{-}+s\right)\right. \\
& \times\left(4\left(k \cdot p_{0}\right)^{2}-k \cdot p_{0}\left(2 k \cdot p_{-}+s\right)+M_{\rho}^{2}\left(2 k \cdot p_{-}+s\right)\right) \lambda_{17}^{V}+\left(8\left(k \cdot p_{0}\right)^{3}-k \cdot p_{0}\right. \\
& \left.\times\left(2 k \cdot p_{-}+s\right)^{2}+M_{\rho}^{2}\left(2 k \cdot p_{-}+s\right)^{2}+2\left(k \cdot p_{0}\right)^{2}\left(2 k \cdot p_{-}-2 M_{\rho}^{2}+s\right)\right) \lambda_{18}^{V} \\
& +8\left(k \cdot p_{0}\right)^{2}\left(k \cdot p_{-}\right) \lambda_{19}^{V}-4\left(k \cdot p_{0}\right)\left(k \cdot p_{-}\right)^{2} \lambda_{19}^{V}-4\left(k \cdot p_{0}\right)\left(k \cdot p_{-}\right) M_{\rho}^{2} \lambda_{19}^{V} \\
& +4\left(k \cdot p_{-}\right)^{2} M_{\rho}^{2} \lambda_{19}^{V}+4\left(k \cdot p_{0}\right)^{2} s \lambda_{19}^{V}-4\left(k \cdot p_{0}\right)\left(k \cdot p_{-}\right) s \lambda_{19}^{V}-2 k \cdot p_{0} M_{\rho}^{2} s \lambda_{19}^{V} \\
& +4 k \cdot p_{-} M_{\rho}^{2} s \lambda_{19}^{V}-\left(k \cdot p_{0}\right) s^{2} \lambda_{19}^{V}+M_{\rho}^{2} s^{2} \lambda_{19}^{V}-8\left(k \cdot p_{0}\right)^{3} \lambda_{21}^{V}+4\left(k \cdot p_{0}\right)^{2}\left(k \cdot p_{-}\right) \lambda_{21}^{V} \\
& +4\left(k \cdot p_{0}\right)^{2} M_{\rho}^{2} \lambda_{21}^{V}-4\left(k \cdot p_{0}\right)\left(k \cdot p_{-}\right) M_{\rho}^{2} \lambda_{21}^{V}+2\left(k \cdot p_{0}\right)^{2} s \lambda_{21}^{V}-2 k \cdot p_{0} M_{\rho}^{2} s \lambda_{21}^{V} \\
& +8\left(k \cdot p_{0}\right)^{3} \lambda_{22}^{V}-4\left(k \cdot p_{0}\right)^{2}\left(k \cdot p_{-}\right) \lambda_{22}^{V}+4\left(k \cdot p_{0}\right)\left(k \cdot p_{-}\right) M_{\rho}^{2} \lambda_{22}^{V}-2\left(k \cdot p_{0}\right)^{2} s \lambda_{22}^{V} \\
& \left.\left.\left.\left.+2 k \cdot p_{0} M_{\rho}^{2} s \lambda_{22}^{V}\right)\right)\right)\right)+\frac{1}{M_{\rho}^{2} D_{\rho}\left[\left(k+p_{-}\right)^{2}\right]}\left(2 G _ { V } \left(2\left(2\left(k \cdot p_{-}\right)^{2}-\left(k \cdot p_{-}\right) s+M_{\rho}^{2} s\right) \kappa_{12}^{V}\right.\right. \\
& \left.+k \cdot p_{-}\left(-2 k \cdot p_{-}+2 M_{\rho}^{2}+s\right) \kappa_{16}^{V}\right)-F_{V}\left(2 \left(2\left(k \cdot p_{-}\right)^{2}+2 k \cdot p_{-} M_{\rho}^{2}-\left(k \cdot p_{-}\right) s\right.\right. \\
& \left.\left.+M_{\rho}^{2} s\right) \kappa_{12}^{V}+k \cdot p_{-}\left(-2 k \cdot p_{-}+4 M_{\rho}^{2}+s\right) \kappa_{16}^{V}\right)+\sqrt{2}\left(k \cdot p _ { - } \kappa _ { 1 6 } ^ { V } \left(-\left(2 k \cdot p_{-}-4 M_{\rho}^{2}-s\right)\right.\right. \\
& \times\left(2 k \cdot p_{0}+s\right) \lambda_{16}^{V}+2\left(\left(2 k \cdot p_{-}-4 M_{\rho}^{2}-s\right)\left(2 k \cdot p_{0}+s\right) \lambda_{17}^{V}-\left(2 k \cdot p_{-}-2 M_{\rho}^{2}-s\right)\right. \\
& \times(P-q)^{2} \lambda_{18}^{V}-4\left(k \cdot p_{0}\right)\left(k \cdot p_{-}\right) \lambda_{19}^{V}+4 k \cdot p_{-} M_{\rho}^{2} \lambda_{19}^{V}+2\left(k \cdot p_{0}\right) s \lambda_{19}^{V}-2\left(k \cdot p_{-}\right) s \lambda_{19}^{V} \\
& +2 M_{\rho}^{2} s \lambda_{19}^{V}+s^{2} \lambda_{19}^{V}+4\left(k \cdot p_{-}\right)^{2} \lambda_{21}^{V}-4 k \cdot p_{-} M_{\rho}^{2} \lambda_{21}^{V}-2\left(k \cdot p_{-}\right) s \lambda_{21}^{V}-4\left(k \cdot p_{-}\right)^{2} \lambda_{22}^{V} \\
& \left.\left.+8 k \cdot p_{-} M_{\rho}^{2} \lambda_{22}^{V}+2\left(k \cdot p_{-}\right) s \lambda_{22}^{V}\right)\right)+2 \kappa_{12}^{V}\left(( 2 k \cdot p _ { 0 } + s ) \left(2\left(k \cdot p_{-}\right)^{2}+2 k \cdot p_{-} M_{\rho}^{2}\right.\right. \\
& \left.-\left(k \cdot p_{-}\right) s+M_{\rho}^{2} s\right) \lambda_{16}^{V}+2\left(\left(2 k \cdot p_{0}+s\right)\left(-2\left(k \cdot p_{-}\right)^{2}-M_{\rho}^{2} s+k \cdot p_{-}\left(-2 M_{\rho}^{2}+s\right)\right) \lambda_{17}^{V}\right. \\
& +(P-q)^{2}\left(2\left(k \cdot p_{-}\right)^{2}-\left(k \cdot p_{-}\right) s+M_{\rho}^{2} s\right) \lambda_{18}^{V}+4\left(k \cdot p_{0}\right)\left(k \cdot p_{-}\right)^{2} \lambda_{19}^{V}-4\left(k \cdot p_{0}\right) \\
& \times\left(k \cdot p_{-}\right) M_{\rho}^{2} \lambda_{19}^{V}+4\left(k \cdot p_{-}\right)^{2} M_{\rho}^{2} \lambda_{19}^{V}-2\left(k \cdot p_{0}\right)\left(k \cdot p_{-}\right) s \lambda_{19}^{V}+2\left(k \cdot p_{-}\right)^{2} s \lambda_{19}^{V} \\
& +2 k \cdot p_{0} M_{\rho}^{2} s \lambda_{19}^{V}-\left(k \cdot p_{-}\right) s^{2} \lambda_{19}^{V}+M_{\rho}^{2} s^{2} \lambda_{19}^{V}-4\left(k \cdot p_{-}\right)^{3} \lambda_{21}^{V}+2\left(k \cdot p_{-}\right)^{2} s \lambda_{21}^{V} \\
& -2 k \cdot p_{-} M_{\rho}^{2} s \lambda_{21}^{V}+4\left(k \cdot p_{-}\right)^{3} \lambda_{22}^{V}+4\left(k \cdot p_{-}\right)^{2} M_{\rho}^{2} \lambda_{22}^{V}-2\left(k \cdot p_{-}\right)^{2} s \lambda_{22}^{V} \\
& \left.\left.\left.\left.\left.+2 k \cdot p_{-} M_{\rho}^{2} s \lambda_{22}^{V}\right)\right)\right)\right)+\frac{4\left(-k \cdot p_{0}+k \cdot p_{-}\right)\left(\kappa_{5}^{A}-\kappa_{6}^{A}+\kappa_{7}^{A}\right)\left(F_{A}-2 \sqrt{2}(P-q)^{2} \lambda_{17}^{A}\right)}{D_{a_{1}}\left[(P-q)^{2}\right]}\right)
\end{aligned}
$$




$$
\begin{aligned}
& a_{3}^{R R}=\frac{1}{3 \sqrt{2} F^{2}}\left(\frac { F _ { V } } { M _ { \rho } ^ { 2 } M _ { \omega } ^ { 2 } D _ { \rho } [ ( k + p _ { 0 } ) ^ { 2 } ] } \left(\sqrt { 2 } F _ { V } \left(M_{\rho}^{2}\left(4 k \cdot p_{0}+2 k \cdot p_{-}+s\right) \kappa_{3}^{V V}\right.\right.\right. \\
& \left.+\left(-4 k \cdot p_{0}+2 k \cdot p_{-}+s\right) D_{\rho}\left[\left(k+p_{0}\right)^{2}\right] \kappa_{4}^{V V}\right)-2\left(\sqrt { 2 } G _ { V } \left(M_{\rho}^{2}\left(2 k \cdot p_{-}+s\right) \kappa_{3}^{V V}\right.\right. \\
& \left.+\left(-4 k \cdot p_{0}+2 k \cdot p_{-}+s\right) D_{\rho}\left[\left(k+p_{0}\right)^{2}\right] \kappa_{4}^{V V}\right)+\left(2 k \cdot p_{0}-M_{\rho}^{2}\right)\left(4 k \cdot p_{0}\right. \\
& \left.-2 k \cdot p_{-}-s\right) \kappa_{4}^{V V}\left(\left(2 k \cdot p_{-}+s\right) \lambda_{16}^{V}-2\left(2 k \cdot p_{-}+s\right) \lambda_{17}^{V}+4 k \cdot p_{0} \lambda_{18}^{V}\right. \\
& \left.+4 k \cdot p_{-} \lambda_{18}^{V}+2 s \lambda_{18}^{V}+4 k \cdot p_{-} \lambda_{19}^{V}+2 s \lambda_{19}^{V}-4 k \cdot p_{0} \lambda_{21}^{V}+4 k \cdot p_{0} \lambda_{22}^{V}\right) \\
& +M_{\rho}^{2} \kappa_{3}^{V V}\left(\left(2 k \cdot p_{-}+s\right)\left(4 k \cdot p_{0}+2 k \cdot p_{-}+s\right) \lambda_{16}^{V}+2\left(-\left(2 k \cdot p_{-}+s\right)\right.\right. \\
& \times\left(4 k \cdot p_{0}+2 k \cdot p_{-}+s\right) \lambda_{17}^{V}+\left(2 k \cdot p_{-}+s\right)(P-q)^{2} \lambda_{18}^{V}+4\left(k \cdot p_{-}\right)^{2} \lambda_{19}^{V} \\
& +4\left(k \cdot p_{-}\right) s \lambda_{19}^{V}+s^{2} \lambda_{19}^{V}-4\left(k \cdot p_{0}\right)\left(k \cdot p_{-}\right) \lambda_{21}^{V}-2\left(k \cdot p_{0}\right) s \lambda_{21}^{V}+8\left(k \cdot p_{0}\right)^{2} \lambda_{22}^{V} \\
& \left.\left.\left.\left.+4\left(k \cdot p_{0}\right)\left(k \cdot p_{-}\right) \lambda_{22}^{V}+2\left(k \cdot p_{0}\right) s \lambda_{22}^{V}\right)\right)\right)\right)+\frac{F_{V}}{M_{\rho}^{2} M_{\omega}^{2} D_{\rho}\left[\left(k+p_{-}\right)^{2}\right]} \\
& \times\left(-\sqrt{2} F_{V}\left(M_{\rho}^{2}\left(6 k \cdot p_{-}+s\right) \kappa_{3}^{V V}+\left(-2 k \cdot p_{-}+s\right) D_{\rho}\left[\left(k+p_{-}\right)^{2}\right] \kappa_{4}^{V V}\right)\right. \\
& +2\left(\sqrt{2} G_{V}\left(M_{\rho}^{2}\left(2 k \cdot p_{-}+s\right) \kappa_{3}^{V V}+\left(-2 k \cdot p_{-}+s\right) D_{\rho}\left[\left(k+p_{-}\right)^{2}\right] \kappa_{4}^{V V}\right)\right. \\
& +\left(2 k \cdot p_{-}-M_{\rho}^{2}\right)\left(2 k \cdot p_{-}-s\right) \kappa_{4}^{V V}\left(\left(2 k \cdot p_{0}+s\right) \lambda_{16}^{V}-2\left(2 k \cdot p_{0}+s\right) \lambda_{17}^{V}\right. \\
& +4 k \cdot p_{0} \lambda_{18}^{V}+4 k \cdot p_{-} \lambda_{18}^{V}+2 s \lambda_{18}^{V}+4 k \cdot p_{0} \lambda_{19}^{V}+2 s \lambda_{19}^{V}-4 k \cdot p_{-} \lambda_{21}^{V} \\
& \left.+4 k \cdot p_{-} \lambda_{22}^{V}\right)+M_{\rho}^{2} \kappa_{3}^{V V}\left(\left(2 k \cdot p_{0}+s\right)\left(6 k \cdot p_{-}+s\right) \lambda_{16}^{V}+2\left(-\left(2 k \cdot p_{0}+s\right)\right.\right. \\
& \times\left(6 k \cdot p_{-}+s\right) \lambda_{17}^{V}+\left(2 k \cdot p_{-}+s\right)(P-q)^{2} \lambda_{18}^{V}-4\left(k \cdot p_{0}\right)\left(k \cdot p_{-}\right) \lambda_{19}^{V} \\
& +8\left(k \cdot p_{-}\right)^{2} \lambda_{19}^{V}+2\left(k \cdot p_{0}\right) s \lambda_{19}^{V}+2\left(k \cdot p_{-}\right) s \lambda_{19}^{V}+s^{2} \lambda_{19}^{V}-4\left(k \cdot p_{-}\right)^{2} \lambda_{21}^{V} \\
& \left.\left.\left.\left.-2\left(k \cdot p_{-}\right) s \lambda_{21}^{V}+12\left(k \cdot p_{-}\right)^{2} \lambda_{22}^{V}+2\left(k \cdot p_{-}\right) s \lambda_{22}^{V}\right)\right)\right)\right)+\frac{4 F_{V}}{M_{\omega}^{2} D_{a_{1}}\left[(P-q)^{2}\right]} \\
& \times\left(\left(-k \cdot p_{0}+k \cdot p_{-}\right)\left(\kappa_{2}^{V A}-\kappa_{3}^{V A}-\kappa_{4}^{V A}\right)\left(\sqrt{2} F_{A}-4(P-q)^{2} \lambda_{17}^{A}\right)\right) \\
& +\frac{8\left(-k \cdot p_{0}+k \cdot p_{-}\right) \kappa_{5}^{V A}\left(\sqrt{2} G_{V}-2 s \lambda_{21}^{V}\right)\left(F_{A}-2 \sqrt{2}(P-q)^{2} \lambda_{17}^{A}\right)}{D_{a_{1}}\left[(P-q)^{2}\right] D_{\rho}[s]} \\
& +\frac{2\left(F_{A}-2 \sqrt{2}(P-q)^{2} \lambda_{17}^{A}\right)}{M_{\rho}^{2} D_{a_{1}}\left[(P-q)^{2}\right] D_{\rho}\left[\left(k+p_{-}\right)^{2}\right]}\left(k \cdot p _ { - } \kappa _ { 1 6 } ^ { V } \left(\left(-8\left(k \cdot p_{0}\right)\left(k \cdot p_{-}\right)+8 k \cdot p_{-} M_{\rho}^{2}\right.\right.\right. \\
& \left.+4\left(k \cdot p_{0}\right) s-4\left(k \cdot p_{-}\right) s+4 M_{\rho}^{2} s+2 s^{2}\right) \lambda_{2}^{V A}+2 M_{\rho}^{2}\left(4 k \cdot p_{0}-2 k \cdot p_{-}+s\right) \lambda_{3}^{V A} \\
& +4\left(k \cdot p_{0}\right)\left(k \cdot p_{-}\right) \lambda_{4}^{V A}+8\left(k \cdot p_{-}\right)^{2} \lambda_{4}^{V A}-8 k \cdot p_{0} M_{\rho}^{2} \lambda_{4}^{V A}-4 k \cdot p_{-} M_{\rho}^{2} \lambda_{4}^{V A} \\
& -2\left(k \cdot p_{0}\right) s \lambda_{4}^{V A}-2\left(k \cdot p_{-}\right) s \lambda_{4}^{V A}-2 M_{\rho}^{2} s \lambda_{4}^{V A}-s^{2} \lambda_{4}^{V A}+8\left(k \cdot p_{0}\right)\left(k \cdot p_{-}\right) \lambda_{5}^{V A} \\
& \left.-16 k \cdot p_{0} M_{\rho}^{2} \lambda_{5}^{V A}-4\left(k \cdot p_{0}\right) s \lambda_{5}^{V A}+4\left(k \cdot p_{-}\right) s \lambda_{5}^{V A}-8 M_{\rho}^{2} s \lambda_{5}^{V A}-2 s^{2} \lambda_{5}^{V A}\right) \\
& +2 \kappa_{12}^{V}\left(2 \left(2 k \cdot p_{0}\left(k \cdot p_{-}-M_{\rho}^{2}\right)\left(2 k \cdot p_{-}-s\right)-\left(k \cdot p_{-}\right) s^{2}+M_{\rho}^{2} s^{2}\right.\right. \\
& \left.+2\left(k \cdot p_{-}\right)^{2}\left(2 M_{\rho}^{2}+s\right)\right) \lambda_{2}^{V A}+2 k \cdot p_{-} M_{\rho}^{2}\left(4 k \cdot p_{0}-2 k \cdot p_{-}+s\right) \lambda_{3}^{V A} \\
& -4\left(k \cdot p_{0}\right)\left(k \cdot p_{-}\right)^{2} \lambda_{4}^{V A}-8\left(k \cdot p_{-}\right)^{3} \lambda_{4}^{V A}-4\left(k \cdot p_{0}\right)\left(k \cdot p_{-}\right) M_{\rho}^{2} \lambda_{4}^{V A} \\
& +4\left(k \cdot p_{-}\right)^{2} M_{\rho}^{2} \lambda_{4}^{V A}+2\left(k \cdot p_{0}\right)\left(k \cdot p_{-}\right) s \lambda_{4}^{V A}+2\left(k \cdot p_{-}\right)^{2} s \lambda_{4}^{V A} \\
& -2 k \cdot p_{0} M_{\rho}^{2} s \lambda_{4}^{V A}-4 k \cdot p_{-} M_{\rho}^{2} s \lambda_{4}^{V A}+\left(k \cdot p_{-}\right) s^{2} \lambda_{4}^{V A}-M_{\rho}^{2} s^{2} \lambda_{4}^{V A} \\
& -8\left(k \cdot p_{0}\right)\left(k \cdot p_{-}\right)^{2} \lambda_{5}^{V A}-8\left(k \cdot p_{0}\right)\left(k \cdot p_{-}\right) M_{\rho}^{2} \lambda_{5}^{V A}+4\left(k \cdot p_{0}\right)\left(k \cdot p_{-}\right) s \lambda_{5}^{V A} \\
& -4\left(k \cdot p_{-}\right)^{2} s \lambda_{5}^{V A}-4 k \cdot p_{0} M_{\rho}^{2} s \lambda_{5}^{V A}-4 k \cdot p_{-} M_{\rho}^{2} s \lambda_{5}^{V A}+2\left(k \cdot p_{-}\right) s^{2} \lambda_{5}^{V A} \\
& \left.\left.-2 M_{\rho}^{2} s^{2} \lambda_{5}^{V A}\right)\right)+\frac{2\left(F_{A}-2 \sqrt{2}(P-q)^{2} \lambda_{17}^{A}\right)}{M_{\rho}^{2} D_{a_{1}}\left[(P-q)^{2}\right] D_{\rho}\left[\left(k+p_{0}\right)^{2}\right]}\left(k \cdot p _ { 0 } \kappa _ { 1 6 } ^ { V } \left(2 \left(4 k \cdot p_{0}-2 k \cdot p_{-}\right.\right.\right.
\end{aligned}
$$




$$
\begin{aligned}
& \left.-2 M_{\rho}^{2}-s\right)\left(2 k \cdot p_{-}+s\right) \lambda_{2}^{V A}-2 M_{\rho}^{2}\left(2 k \cdot p_{-}+s\right) \lambda_{3}^{V A}-16\left(k \cdot p_{0}\right)^{2} \lambda_{4}^{V A} \\
& +4\left(k \cdot p_{-}\right)^{2} \lambda_{4}^{V A}+8 k \cdot p_{0} M_{\rho}^{2} \lambda_{4}^{V A}+4 k \cdot p_{-} M_{\rho}^{2} \lambda_{4}^{V A}+4\left(k \cdot p_{-}\right) s \lambda_{4}^{V A} \\
& +2 M_{\rho}^{2} s \lambda_{4}^{V A}+s^{2} \lambda_{4}^{V A}-16\left(k \cdot p_{0}\right)\left(k \cdot p_{-}\right) \lambda_{5}^{V A}+8\left(k \cdot p_{-}\right)^{2} \lambda_{5}^{V A} \\
& \left.+16 k \cdot p_{-} M_{\rho}^{2} \lambda_{5}^{V A}-8\left(k \cdot p_{0}\right) s \lambda_{5}^{V A}+8\left(k \cdot p_{-}\right) s \lambda_{5}^{V A}+8 M_{\rho}^{2} s \lambda_{5}^{V A}+2 s^{2} \lambda_{5}^{V A}\right) \\
& +2 \kappa_{12}^{V}\left(-2\left(2 k \cdot p_{-}+s\right)\left(4\left(k \cdot p_{0}\right)^{2}+M_{\rho}^{2}\left(2 k \cdot p_{-}+s\right)-k \cdot p_{0}\right.\right. \\
& \left.\times\left(2 k \cdot p_{-}+2 M_{\rho}^{2}+s\right)\right) \lambda_{2}^{V A}-2 k \cdot p_{0} M_{\rho}^{2}\left(2 k \cdot p_{-}+s\right) \lambda_{3}^{V A}+16\left(k \cdot p_{0}\right)^{3} \lambda_{4}^{V A} \\
& -4\left(k \cdot p_{0}\right)\left(k \cdot p_{-}\right)^{2} \lambda_{4}^{V A}-8\left(k \cdot p_{0}\right)^{2} M_{\rho}^{2} \lambda_{4}^{V A}+4\left(k \cdot p_{0}\right)\left(k \cdot p_{-}\right) M_{\rho}^{2} \lambda_{4}^{V A} \\
& +4\left(k \cdot p_{-}\right)^{2} M_{\rho}^{2} \lambda_{4}^{V A}-4\left(k \cdot p_{0}\right)\left(k \cdot p_{-}\right) s \lambda_{4}^{V A}+2 k \cdot p_{0} M_{\rho}^{2} s \lambda_{4}^{V A} \\
& +4 k \cdot p_{-} M_{\rho}^{2} s \lambda_{4}^{V A}-\left(k \cdot p_{0}\right) s^{2} \lambda_{4}^{V A}+M_{\rho}^{2} s^{2} \lambda_{4}^{V A}+16\left(k \cdot p_{0}\right)^{2}\left(k \cdot p_{-}\right) \lambda_{5}^{V A} \\
& -8\left(k \cdot p_{0}\right)\left(k \cdot p_{-}\right)^{2} \lambda_{5}^{V A}+8\left(k \cdot p_{-}\right)^{2} M_{\rho}^{2} \lambda_{5}^{V A}+8\left(k \cdot p_{0}\right)^{2} s \lambda_{5}^{V A} \\
& \left.\left.\left.-8\left(k \cdot p_{0}\right)\left(k \cdot p_{-}\right) s \lambda_{5}^{V A}+8 k \cdot p_{-} M_{\rho}^{2} s \lambda_{5}^{V A}-2\left(k \cdot p_{0}\right) s^{2} \lambda_{5}^{V A}+2 M_{\rho}^{2} s^{2} \lambda_{5}^{V A}\right)\right)\right)
\end{aligned}
$$

$$
\begin{aligned}
a_{3}^{R R R}= & \frac{F_{V}\left(\sqrt{2} F_{A}-4(P-q)^{2} \lambda_{17}^{A}\right)}{3 \sqrt{2} F^{2} M_{\rho}^{2} M_{\omega}^{2} D_{a_{1}}\left[(P-q)^{2}\right] D_{\rho}\left[\left(k+p_{0}\right)^{2}\right] D_{\rho}\left[\left(k+p_{-}\right)^{2}\right]}\left(-M_{\rho}^{2}\left(2 k \cdot p_{-}+s\right)\right. \\
& \times D_{\rho}\left[\left(k+p_{-}\right)^{2}\right] \kappa_{3}^{V V}\left(2\left(2 k \cdot p_{-}+s\right) \lambda_{2}^{V A}+4 k \cdot p_{0} \lambda_{3}^{V A}-\left(4 k \cdot p_{0}+2 k \cdot p_{-}+s\right)\right. \\
& \left.\times\left(\lambda_{4}^{V A}+2 \lambda_{5}^{V A}\right)\right)-D_{\rho}\left[\left(k+p_{0}\right)^{2}\right]\left(2\left(k \cdot p_{0}-k \cdot p_{-}\right) D_{\rho}\left[\left(k+p_{-}\right)^{2}\right] \kappa_{4}^{V V}\right. \\
& \times\left(-2\left(2 k \cdot p_{-}+3 s\right) \lambda_{2}^{V A}+\left(8 k \cdot p_{0}+6 k \cdot p_{-}+s\right) \lambda_{4}^{V A}+2\left(2 k \cdot p_{-}+3 s\right) \lambda_{5}^{V A}\right) \\
& +M_{\rho}^{2} \kappa_{3}^{V V}\left(2\left(4\left(k \cdot p_{0}\right)\left(k \cdot p_{-}\right)-8\left(k \cdot p_{-}\right)^{2}-2\left(k \cdot p_{0}\right) s-2\left(k \cdot p_{-}\right) s-s^{2}\right) \lambda_{2}^{V A}\right. \\
& \left.\left.\left.-4 k \cdot p_{-}\left(4 k \cdot p_{0}-2 k \cdot p_{-}+s\right) \lambda_{3}^{V A}+\left(2 k \cdot p_{0}+s\right)\left(6 k \cdot p_{-}+s\right)\left(\lambda_{4}^{V A}+2 \lambda_{5}^{V A}\right)\right)\right)\right)
\end{aligned}
$$

$$
\begin{aligned}
a_{4}^{R}= & \frac{4 \sqrt{2}}{3 F^{2} M_{\rho}^{2}}\left(\frac { 1 } { D _ { \rho } [ ( k + p _ { 0 } ) ^ { 2 } ] } \left(G_{V}\left(4\left(k \cdot p_{0}-M_{\rho}^{2}\right) \kappa_{12}^{V}-2 k \cdot p_{0} \kappa_{16}^{V}\right)\right.\right. \\
& +F_{V}\left(-2\left(k \cdot p_{0}-M_{\rho}^{2}\right) \kappa_{12}^{V}+k \cdot p_{0} \kappa_{16}^{V}\right)+\sqrt{2}\left(-k \cdot p_{0} \kappa_{16}^{V}\left(\left(2 k \cdot p_{-}+s\right) \lambda_{16}^{V}\right.\right. \\
& -2\left(2 k \cdot p_{-}+s\right) \lambda_{17}^{V}+4 k \cdot p_{0} \lambda_{18}^{V}+4 k \cdot p_{-} \lambda_{18}^{V}+2 s \lambda_{18}^{V}+4 k \cdot p_{-} \lambda_{19}^{V}+4 M_{\rho}^{2} \lambda_{19}^{V} \\
& \left.+2 s \lambda_{19}^{V}-4 k \cdot p_{0} \lambda_{21}^{V}+4 k \cdot p_{0} \lambda_{22}^{V}\right)+2 \kappa_{12}^{V}\left(\left(k \cdot p_{0}-M_{\rho}^{2}\right)\left(2 k \cdot p_{-}+s\right) \lambda_{16}^{V}\right. \\
& -2\left(k \cdot p_{0}-M_{\rho}^{2}\right)\left(2 k \cdot p_{-}+s\right) \lambda_{17}^{V}+4\left(k \cdot p_{0}\right)^{2} \lambda_{18}^{V}+4\left(k \cdot p_{0}\right)\left(k \cdot p_{-}\right) \lambda_{18}^{V} \\
& -4 k \cdot p_{0} M_{\rho}^{2} \lambda_{18}^{V}-4 k \cdot p_{-} M_{\rho}^{2} \lambda_{18}^{V}+2\left(k \cdot p_{0}\right) s \lambda_{18}^{V}-2 M_{\rho}^{2} s \lambda_{18}^{V}+4\left(k \cdot p_{0}\right)\left(k \cdot p_{-}\right) \lambda_{19}^{V} \\
& -4 k \cdot p_{0} M_{\rho}^{2} \lambda_{19}^{V}-4 k \cdot p_{-} M_{\rho}^{2} \lambda_{19}^{V}+2\left(k \cdot p_{0}\right) s \lambda_{19}^{V}-2 M_{\rho}^{2} s \lambda_{19}^{V}-4\left(k \cdot p_{0}\right)^{2} \lambda_{21}^{V} \\
& \left.\left.\left.+4 k \cdot p_{0} M_{\rho}^{2} \lambda_{21}^{V}+4\left(k \cdot p_{0}\right)^{2} \lambda_{22}^{V}-4 k \cdot p_{0} M_{\rho}^{2} \lambda_{22}^{V}\right)\right)\right)+\frac{1}{D_{\rho}\left[\left(k+p_{-}\right)^{2}\right]} \\
& \times\left(F_{V}\left(2\left(k \cdot p_{-}-M_{\rho}^{2}\right) \kappa_{12}^{V}-k \cdot p_{-} \kappa_{16}^{V}\right)+G_{V}\left(-4\left(k \cdot p_{-}-M_{\rho}^{2}\right) \kappa_{12}^{V}+2 k \cdot p_{-} \kappa_{16}^{V}\right)\right. \\
& +\sqrt{2}\left(k \cdot p _ { - } \kappa _ { 1 6 } ^ { V } \left(\left(2 k \cdot p_{0}+s\right) \lambda_{16}^{V}-2\left(2 k \cdot p_{0}+s\right) \lambda_{17}^{V}+4 k \cdot p_{0} \lambda_{18}^{V}+4 k \cdot p_{-} \lambda_{18}^{V}\right.\right. \\
& \left.+2 s \lambda_{18}^{V}+4 k \cdot p_{0} \lambda_{19}^{V}+4 M_{\rho}^{2} \lambda_{19}^{V}+2 s \lambda_{19}^{V}-4 k \cdot p_{-} \lambda_{21}^{V}+4 k \cdot p_{-} \lambda_{22}^{V}\right) \\
& +\kappa_{12}^{V}\left(-2\left(k \cdot p_{-}-M_{\rho}^{2}\right)\left(2 k \cdot p_{0}+s\right) \lambda_{16}^{V}+4\left(k \cdot p_{-}-M_{\rho}^{2}\right)\left(2 k \cdot p_{0}+s\right) \lambda_{17}^{V}\right. \\
& -4\left(k \cdot p_{-}-M_{\rho}^{2}\right)(P-q)^{2} \lambda_{18}^{V}-8\left(k \cdot p_{0}\right)\left(k \cdot p_{-}\right) \lambda_{19}^{V}+8 k \cdot p_{0} M_{\rho}^{2} \lambda_{19}^{V}+8 k \cdot p_{-} M_{\rho}^{2} \lambda_{19}^{V} \\
& -4\left(k \cdot p_{-}\right) s \lambda_{19}^{V}+4 M_{\rho}^{2} s \lambda_{19}^{V}+8\left(k \cdot p_{-}\right)^{2} \lambda_{21}^{V}-8 k \cdot p_{-} M_{\rho}^{2} \lambda_{21}^{V}-8\left(k \cdot p_{-}\right)^{2} \lambda_{22}^{V} \\
& \left.\left.\left.\left.+8 k \cdot p_{-} M_{\rho}^{2} \lambda_{22}^{V}\right)\right)\right)\right)
\end{aligned}
$$




$$
\begin{aligned}
& a_{4}^{R R}=\frac{2 \sqrt{2}}{3 F^{2} M_{\rho}^{2}}\left(\frac { F _ { V } } { M _ { \omega } ^ { 2 } D _ { \rho } [ ( k + p _ { 0 } ) ^ { 2 } ] } \left(\sqrt{2} F_{V}\left(M_{\rho}^{2} \kappa_{3}^{V V}+D_{\rho}\left[\left(k+p_{0}\right)^{2}\right] \kappa_{4}^{V V}\right)\right.\right. \\
& -2 \sqrt{2} G_{V}\left(M_{\rho}^{2} \kappa_{3}^{V V}+D_{\rho}\left[\left(k+p_{0}\right)^{2}\right] \kappa_{4}^{V V}\right)+2\left(2 k \cdot p_{0}-M_{\rho}^{2}\right) \kappa_{4}^{V V} \\
& \times\left(\left(2 k \cdot p_{-}+s\right) \lambda_{16}^{V}-2\left(2 k \cdot p_{-}+s\right) \lambda_{17}^{V}+4 k \cdot p_{0} \lambda_{18}^{V}+4 k \cdot p_{-} \lambda_{18}^{V}+2 s \lambda_{18}^{V}\right. \\
& \left.+4 k \cdot p_{-} \lambda_{19}^{V}+2 s \lambda_{19}^{V}-4 k \cdot p_{0} \lambda_{21}^{V}+4 k \cdot p_{0} \lambda_{22}^{V}\right)-2 M_{\rho}^{2} \kappa_{3}^{V V}\left(\left(2 k \cdot p_{-}+s\right) \lambda_{16}^{V}\right. \\
& -2\left(2 k \cdot p_{-}+s\right) \lambda_{17}^{V}+4 k \cdot p_{0} \lambda_{18}^{V}+4 k \cdot p_{-} \lambda_{18}^{V}+2 s \lambda_{18}^{V}+8 k \cdot p_{0} \lambda_{19}^{V}+4 k \cdot p_{-} \lambda_{19}^{V} \\
& \left.\left.+2 s \lambda_{19}^{V}-4 k \cdot p_{0} \lambda_{21}^{V}+4 k \cdot p_{0} \lambda_{22}^{V}\right)\right)+\frac{F_{V}}{M_{\omega}^{2} D_{\rho}\left[\left(k+p_{-}\right)^{2}\right]}\left(-\sqrt{2} F_{V}\left(M_{\rho}^{2} \kappa_{3}^{V V}\right.\right. \\
& \left.+D_{\rho}\left[\left(k+p_{-}\right)^{2}\right] \kappa_{4}^{V V}\right)+2 \sqrt{2} G_{V}\left(M_{\rho}^{2} \kappa_{3}^{V V}+D_{\rho}\left[\left(k+p_{-}\right)^{2}\right] \kappa_{4}^{V V}\right) \\
& -2\left(2 k \cdot p_{-}-M_{\rho}^{2}\right) \kappa_{4}^{V V}\left(\left(2 k \cdot p_{0}+s\right) \lambda_{16}^{V}-2\left(2 k \cdot p_{0}+s\right) \lambda_{17}^{V}+4 k \cdot p_{0} \lambda_{18}^{V}\right. \\
& \left.+4 k \cdot p_{-} \lambda_{18}^{V}+2 s \lambda_{18}^{V}+4 k \cdot p_{0} \lambda_{19}^{V}+2 s \lambda_{19}^{V}-4 k \cdot p_{-} \lambda_{21}^{V}+4 k \cdot p_{-} \lambda_{22}^{V}\right) \\
& +2 M_{\rho}^{2} \kappa_{3}^{V V}\left(\left(2 k \cdot p_{0}+s\right) \lambda_{16}^{V}-2\left(2 k \cdot p_{0}+s\right) \lambda_{17}^{V}+4 k \cdot p_{0} \lambda_{18}^{V}+4 k \cdot p_{-} \lambda_{18}^{V}\right. \\
& \left.\left.+2 s \lambda_{18}^{V}+4 k \cdot p_{0} \lambda_{19}^{V}+8 k \cdot p_{-} \lambda_{19}^{V}+2 s \lambda_{19}^{V}-4 k \cdot p_{-} \lambda_{21}^{V}+4 k \cdot p_{-} \lambda_{22}^{V}\right)\right) \\
& -\frac{2\left(F_{A}-2 \sqrt{2}(P-q)^{2} \lambda_{17}^{A}\right)}{D_{a_{1}}\left[(P-q)^{2}\right] D_{\rho}\left[\left(k+p_{-}\right)^{2}\right]}\left(k \cdot p _ { - } \kappa _ { 1 6 } ^ { V } \left(-2\left(2 k \cdot p_{0}+2 M_{\rho}^{2}+s\right) \lambda_{2}^{V A}\right.\right. \\
& \left.+2 M_{\rho}^{2} \lambda_{3}^{V A}+2 k \cdot p_{0} \lambda_{4}^{V A}+4 k \cdot p_{-} \lambda_{4}^{V A}-2 M_{\rho}^{2} \lambda_{4}^{V A}+s \lambda_{4}^{V A}+4 k \cdot p_{0} \lambda_{5}^{V A}+2 s \lambda_{5}^{V A}\right) \\
& +2 \kappa_{12}^{V}\left(2\left(2\left(k \cdot p_{0}\right)\left(k \cdot p_{-}\right)-2 k \cdot p_{0} M_{\rho}^{2}-2 k \cdot p_{-} M_{\rho}^{2}+\left(k \cdot p_{-}\right) s-M_{\rho}^{2} s\right) \lambda_{2}^{V A}\right. \\
& +2 k \cdot p_{-} M_{\rho}^{2} \lambda_{3}^{V A}-2\left(k \cdot p_{0}\right)\left(k \cdot p_{-}\right) \lambda_{4}^{V A}-4\left(k \cdot p_{-}\right)^{2} \lambda_{4}^{V A}+2 k \cdot p_{0} M_{\rho}^{2} \lambda_{4}^{V A} \\
& +2 k \cdot p_{-} M_{\rho}^{2} \lambda_{4}^{V A}-\left(k \cdot p_{-}\right) s \lambda_{4}^{V A}+M_{\rho}^{2} s \lambda_{4}^{V A}-4\left(k \cdot p_{0}\right)\left(k \cdot p_{-}\right) \lambda_{5}^{V A} \\
& \left.\left.+4 k \cdot p_{0} M_{\rho}^{2} \lambda_{5}^{V A}-2\left(k \cdot p_{-}\right) s \lambda_{5}^{V A}+2 M_{\rho}^{2} s \lambda_{5}^{V A}\right)\right)+\frac{2\left(F_{A}-2 \sqrt{2}(P-q)^{2} \lambda_{17}^{A}\right)}{D_{a_{1}}\left[(P-q)^{2}\right] D_{\rho}\left[\left(k+p_{0}\right)^{2}\right]} \\
& \times\left(k \cdot p _ { 0 } \kappa _ { 1 6 } ^ { V } \left(-2\left(2 k \cdot p_{-}+2 M_{\rho}^{2}+s\right) \lambda_{2}^{V A}+2 M_{\rho}^{2} \lambda_{3}^{V A}+4 k \cdot p_{0} \lambda_{4}^{V A}+2 k \cdot p_{-} \lambda_{4}^{V A}\right.\right. \\
& \left.-2 M_{\rho}^{2} \lambda_{4}^{V A}+s \lambda_{4}^{V A}+4 k \cdot p_{-} \lambda_{5}^{V A}+2 s \lambda_{5}^{V A}\right)+\kappa_{12}^{V}\left(4 \left(-M_{\rho}^{2}\left(2 k \cdot p_{-}+s\right)\right.\right. \\
& \left.+k \cdot p_{0}\left(2 k \cdot p_{-}-2 M_{\rho}^{2}+s\right)\right) \lambda_{2}^{V A}+4 k \cdot p_{0} M_{\rho}^{2} \lambda_{3}^{V A}-8\left(k \cdot p_{0}\right)^{2} \lambda_{4}^{V A} \\
& -4\left(k \cdot p_{0}\right)\left(k \cdot p_{-}\right) \lambda_{4}^{V A}+4 k \cdot p_{0} M_{\rho}^{2} \lambda_{4}^{V A}+4 k \cdot p_{-} M_{\rho}^{2} \lambda_{4}^{V A}-2\left(k \cdot p_{0}\right) s \lambda_{4}^{V A} \\
& +2 M_{\rho}^{2} s \lambda_{4}^{V A}-8\left(k \cdot p_{0}\right)\left(k \cdot p_{-}\right) \lambda_{5}^{V A}+8 k \cdot p_{-} M_{\rho}^{2} \lambda_{5}^{V A}-4\left(k \cdot p_{0}\right) s \lambda_{5}^{V A} \\
& \left.\left.\left.+4 M_{\rho}^{2} s \lambda_{5}^{V A}\right)\right)\right) \\
& a_{4}^{R R R}=-\frac{4 \sqrt{2} F_{V}\left(k \cdot p_{0}-k \cdot p_{-}\right)\left(\sqrt{2} F_{A}-4(P-q)^{2} \lambda_{17}^{A}\right)}{3 F^{2} M_{\rho}^{2} M_{\omega}^{2} D_{a_{1}}\left[(P-q)^{2}\right] D_{\rho}\left[\left(k+p_{0}\right)^{2}\right] D_{\rho}\left[\left(k+p_{-}\right)^{2}\right]} \\
& \times\left(-D_{\rho}\left[\left(k+p_{0}\right)^{2}\right] D_{\rho}\left[\left(k+p_{-}\right)^{2}\right] \kappa_{4}^{V V}\left(2 \lambda_{2}^{V A}+\lambda_{4}^{V A}-2 \lambda_{5}^{V A}\right)\right. \\
& \left.+M_{\rho}^{2} \kappa_{3}^{V V}\left(2\left(M_{\rho}^{2}+(P-q)^{2}\right) \lambda_{2}^{V A}-2 M_{\rho}^{2} \lambda_{3}^{V A}+D_{\rho}\left[(P-q)^{2}\right]\left(\lambda_{4}^{V A}+2 \lambda_{5}^{V A}\right)\right)\right)
\end{aligned}
$$


[1] J. Grange et al., Muon (g-2) technical design report, arXiv:1501.06858.

[2] T. Aoyama et al., The anomalous magnetic moment of the muon in the standard model, arXiv:2006.04822.

[3] M. Davier, A. Hoecker, B. Malaescu, and Z. Zhang, Reevaluation of the hadronic vacuum polarisation contributions to the standard model predictions of the muon $g-2$ and $\alpha\left(m_{Z}^{2}\right)$ using newest hadronic cross-section data, Eur. Phys. J. C 77, 827 (2017).

[4] A. Keshavarzi, D. Nomura, and T. Teubner, Muon $g-2$ and $\alpha\left(M_{Z}^{2}\right)$ : a new data-based analysis, Phys. Rev. D 97, 114025 (2018).

[5] G. Colangelo, M. Hoferichter, and P. Stoffer, Two-pion contribution to hadronic vacuum polarization, J. High Energy Phys. 02 (2019) 006.

[6] M. Hoferichter, B.-L. Hoid, and B. Kubis, Three-pion contribution to hadronic vacuum polarization, J. High Energy Phys. 08 (2019) 137.

[7] M. Davier, A. Hoecker, B. Malaescu, and Z. Zhang, A new evaluation of the hadronic vacuum polarisation contributions to the muon anomalous magnetic moment and to $\alpha\left(m_{Z}^{2}\right)$, Eur. Phys. J. C 80, 241 (2020).

[8] A. Keshavarzi, D. Nomura, and T. Teubner, The $g-2$ of charged leptons, $\alpha\left(M_{Z}^{2}\right)$ and the hyperfine splitting of muonium, Phys. Rev. D 101, 014029 (2020).

[9] A. Kurz, T. Liu, P. Marquard, and M. Steinhauser, Hadronic contribution to the muon anomalous magnetic moment to next-to-next-to-leading order, Phys. Lett. B 734, 144 (2014).

[10] B. Chakraborty et al., Strong-Isospin-Breaking Correction to the Muon Anomalous Magnetic Moment from Lattice QCD at the Physical Point, Phys. Rev. Lett. 120, 152001 (2018).

[11] Sz. Borsanyi et al., Hadronic Vacuum Polarization Contribution to the Anomalous Magnetic Moments of Leptons from First Principles, Phys. Rev. Lett. 121, 022002 (2018).

[12] T. Blum, P. A. Boyle, V. Gülpers, T. Izubuchi, L. Jin, C. Jung, A. Jüttner, C. Lehner, A. Portelli, and J. T. Tsang, Calculation of the Hadronic Vacuum Polarization Contribution to the Muon Anomalous Magnetic Moment, Phys. Rev. Lett. 121, 022003 (2018).

[13] D. Giusti, V. Lubicz, G. Martinelli, F. Sanfilippo, and S. Simula, Electromagnetic and strong isospin-breaking corrections to the muon $g-2$ from Lattice QCD + QED, Phys. Rev. D 99, 114502 (2019).

[14] E. Shintani and Y. Kuramashi, Study of systematic uncertainties in hadronic vacuum polarization contribution to muon $g-2$ with $2+1$ flavor lattice QCD, Phys. Rev. D 100, 034517 (2019).

[15] C. T. H. Davies et al., Hadronic-vacuum-polarization contribution to the muon's anomalous magnetic moment from four-flavor lattice QCD, Phys. Rev. D 101, 034512 (2020).

[16] A. Gérardin, M. Cè, G. von Hippel, B. Hörz, H. B. Meyer, D. Mohler, K. Ottnad, J. Wilhelm, and H. Wittig, The leading hadronic contribution to $(g-2)_{\mu}$ from lattice QCD with $N_{\mathrm{f}}=2+1$ flavours of $\mathrm{O}(a)$ improved Wilson quarks, Phys. Rev. D 100, 014510 (2019).

[17] C. Aubin, T. Blum, C. Tu, M. Golterman, C. Jung, and S. Peris, Light quark vacuum polarization at the physical point and contribution to the muon $g-2$, Phys. Rev. D 101, 014503 (2020).

[18] D. Giusti and S. Simula, Lepton anomalous magnetic moments in lattice QCD + QED, Proc. Sci., LATTICE2019 (2019) 104 [arXiv:1910.03874].

[19] K. Melnikov and A. Vainshtein, Hadronic light-by-light scattering contribution to the muon anomalous magnetic moment revisited, Phys. Rev. D 70, 113006 (2004).

[20] P. Masjuan and P. Sánchez-Puertas, Pseudoscalar-pole contribution to the $\left(g_{\mu}-2\right)$ : A rational approach, Phys. Rev. D 95, 054026 (2017).

[21] G. Colangelo, M. Hoferichter, M. Procura, and P. Stoffer, Dispersion relation for hadronic light-by-light scattering: two-pion contributions, J. High Energy Phys. 04 (2017) 161.

[22] M. Hoferichter, B.-L. Hoid, B. Kubis, S. Leupold, and S. P. Schneider, Dispersion relation for hadronic light-by-light scattering: pion pole, J. High Energy Phys. 10 (2018) 141.

[23] A. Gérardin, H. B. Meyer, and A. Nyffeler, Lattice calculation of the pion transition form factor with $N_{f}=2+1$ Wilson quarks, Phys. Rev. D 100, 034520 (2019).

[24] J. Bijnens, N. Hermansson-Truedsson, and A. RodríguezSánchez, Short-distance constraints for the HLbL contribution to the muon anomalous magnetic moment, Phys. Lett. B 798, 134994 (2019).

[25] G. Colangelo, F. Hagelstein, M. Hoferichter, L. Laub, and P. Stoffer, Longitudinal short-distance constraints for the hadronic light-by-light contribution to $(g-2)_{\mu}$ with large- $N_{c}$ Regge models, J. High Energy Phys. 03 (2020) 101.

[26] V. Pauk and M. Vanderhaeghen, Single meson contributions to the muon's anomalous magnetic moment, Eur. Phys. J. C 74, 3008 (2014).

[27] I. Danilkin and M. Vanderhaeghen, Light-by-light scattering sum rules in light of new data, Phys. Rev. D 95, 014019 (2017).

[28] F. Jegerlehner, The anomalous magnetic moment of the muon, Springer Tracts Mod. Phys. 274, 1 (2017).

[29] M. Knecht, S. Narison, A. Rabemananjara, and D. Rabetiarivony, Scalar meson contributions to $a_{\mu}$ from hadronic light-by-light scattering, Phys. Lett. B 787, 111 (2018).

[30] G. Eichmann, C. S. Fischer, and R. Williams, Kaon-box contribution to the anomalous magnetic moment of the muon, Phys. Rev. D 101, 054015 (2020).

[31] P. Roig and P. Sánchez-Puertas, Axial-vector exchange contribution to the hadronic light-by-light piece of the muon anomalous magnetic moment, Phys. Rev. D 101, 074019 (1910).

[32] G. Colangelo, M. Hoferichter, A. Nyffeler, M. Passera, and P. Stoffer, Remarks on higher-order hadronic corrections to the muon $g-2$, Phys. Lett. B 735, 90 (2014).

[33] T. Blum, N. Christ, M. Hayakawa, T. Izubuchi, L. Jin, C. Jung, and C. Lehner, The Hadronic Light-by-Light Scattering Contribution to the Muon Anomalous Magnetic Moment from Lattice QCD, Phys. Rev. Lett. 124, 132002 (2020).

[34] T. Aoyama, M. Hayakawa, T. Kinoshita, and M. Nio, Complete Tenth-Order QED Contribution to the Muon g-2, Phys. Rev. Lett. 109, 111808 (2012). 
[35] T. Aoyama, T. Kinoshita, and M. Nio, Theory of the anomalous magnetic moment of the electron, Atoms 7, 28 (2019).

[36] A. Czarnecki, W. J. Marciano, and A. Vainshtein, Refinements in electroweak contributions to the muon anomalous magnetic moment, Phys. Rev. D 67, 073006 (2003); Erratum, Phys. Rev. D 73, 119901 (2006).

[37] C. Gnendiger, D. Stöckinger, and H. Stöckinger-Kim, The electroweak contributions to $(g-2)_{\mu}$ after the Higgs boson mass measurement, Phys. Rev. D 88, 053005 (2013).

[38] M. Knecht, On some short-distance properties of the fourth-rank hadronic vacuum polarization tensor and the anomalous magnetic moment of the muon, J. High Energy Phys. 08 (2020) 056.

[39] P. Masjuan, P. Roig, and P. Sánchez-Puertas, A different viewpoint on the Hadronic light-by-light tensor shortdistance constraints, arXiv:2005.11761.

[40] J. Lüdtke and M. Procura, Effects of longitudinal shortdistance constraints on the hadronic light-by-light contribution to the Muon $g-2$, arXiv:2006.00007.

[41] B.-L. Hoid, M. Hoferichter, and B. Kubis, Hadronic vacuum polarization and vector-meson resonance parameters from $e^{+} e^{-} \rightarrow \pi^{0} \gamma$, arXiv:2007.12696.

[42] B. Ananthanarayan, I. Caprini, and D. Das, Test of analyticity and unitarity for the pion form-factor data around the $\rho$ resonance, arXiv:2008.00669.

[43] C. Aubin, T. Blum, M. Golterman, and S. Peris, On the application of effective field theory to finite-volume effects in $a_{\mu}^{\mathrm{HVP}}$, arXiv:2008.03809.

[44] J. Bijnens, N. Hermansson-Truedsson, L. Laub, and A. Rodríguez-Sánchez, Short-distance HLbL contributions to the muon anomalous magnetic moment beyond perturbation theory, arXiv:2008.13487.

[45] G. W. Bennett et al., Final Report of the muon E821 anomalous magnetic moment measurement at BNL, Phys. Rev. D 73, 072003 (2006).

[46] M. Abe et al., A new approach for measuring the muon anomalous magnetic moment and electric dipole moment, Prog. Theor. Exp. Phys. 2019, 053C02 (2019).

[47] V. M. Aul'chenko et al., Measurement of the pion formfactor in the range 1.04-GeV to $1.38-\mathrm{GeV}$ with the CMD-2 detector, Pis'ma Zh. Eksp. Teor. Fiz. 82, 841 (2005) [JETP Lett. 82, 743 (2005)].

[48] M. N. Achasov et al., Update of the e+e- $\rightarrow$ pi+ pi- crosssection measured by SND detector in the energy region $400-\mathrm{MeV}<\mathrm{s}^{* *}(1 / 2)<$ file1000-MeV, Zh. Eksp. Teor. Fiz. 130, 437 (2006) [J. Exp. Theor. Phys. 103, 380 (2006)].

[49] V. M. Aul'chenko et al., Measurement of the e+ e- $\rightarrow$ pit pi- cross section with the CMD-2 detector in the 370-520-MeV c.m. energy range, Pis'ma Zh. Eksp. Teor. Fiz. 84, 491 (2006) [JETP Lett. 84, 413 (2006)].

[50] R. R. Akhmetshin et al., High-statistics measurement of the pion form factor in the rho-meson energy range with the CMD-2 detector, Phys. Lett. B 648, 28 (2007).

[51] F. Ambrosino et al., Measurement of $\sigma\left(e^{+} e^{-} \rightarrow \pi^{+} \pi^{-} \gamma(\gamma)\right.$ and the dipion contribution to the muon anomaly with the KLOE detector, Phys. Lett. B 670, 285 (2009).

[52] B. Aubert et al., Precise Measurement of the e+ e- $\longrightarrow$ pi+ pi- (gamma) Cross Section with the Initial State Radiation Method at BABAR, Phys. Rev. Lett. 103, 231801 (2009).
[53] F. Ambrosino et al., Measurement of $\sigma\left(e^{+} e^{-} \rightarrow \pi^{+} \pi^{-}\right)$ from threshold to $0.85 \mathrm{GeV}^{2}$ using initial state radiation with the KLOE detector, Phys. Lett. B 700, 102 (2011).

[54] J. P. Lees et al., Precise measurement of the $e^{+} e^{-} \rightarrow$ $\pi^{+} \pi^{-}(\gamma)$ cross section with the initial-state radiation method at BABAR, Phys. Rev. D 86, 032013 (2012).

[55] M. Ablikim et al., Measurement of the $e^{+} e^{-} \rightarrow \pi^{+} \pi^{-}$ cross section between 600 and $900 \mathrm{MeV}$ using initial state radiation, Phys. Lett. B 753, 629 (2016).

[56] A. Anastasi et al., Combination of KLOE $\sigma\left(e^{+} e^{-} \rightarrow\right.$ $\left.\pi^{+} \pi^{-} \gamma(\gamma)\right)$ measurements and determination of $a_{\mu}^{\pi^{+} \pi^{-}}$in the energy range $0.10<s<0.95 \mathrm{GeV}^{2}$, J. High Energy Phys. 03 (2018) 173.

[57] S. J. Brodsky and E. De Rafael, Suggested Boson-Lepton pair couplings and the anomalous magnetic moment of the muon, Phys. Rev. 168, 1620 (1968).

[58] B.E. Lautrup and E. De Rafael, Calculation of the sixth-order contribution from the fourth-order vacuum polarization to the difference of the anomalous magnetic moments of muon and electron, Phys. Rev. 174, 1835 (1968).

[59] R. Alemany, M. Davier, and A. Hocker, Improved determination of the hadronic contribution to the muon (g-2) and to alpha $(\mathrm{M}(\mathrm{z}))$ using new data from hadronic tau decays, Eur. Phys. J. C 2, 123 (1998).

[60] S. Borsanyi et al., Leading-order hadronic vacuum polarization contribution to the muon magnetic momentfrom lattice QCD, arXiv:2002.12347.

[61] V. Cirigliano, G. Ecker, and H. Neufeld, Isospin violation and the magnetic moment of the muon, Phys. Lett. B 513, 361 (2001).

[62] V. Cirigliano, G. Ecker, and H. Neufeld, Radiative tau decay and the magnetic moment of the muon, J. High Energy Phys. 08 (2002) 002.

[63] G. Ecker, J. Gasser, A. Pich, and E. de Rafael, The role of resonances in chiral perturbation theory, Nucl. Phys. B321, 311 (1989).

[64] G. Ecker, J. Gasser, H. Leutwyler, A. Pich, and E. de Rafael, Chiral Lagrangians for massive spin 1 fields, Phys. Lett. B 223, 425 (1989).

[65] F. Flores-Báez, A. Flores-Tlalpa, G. L. Castro, and G. T. Sánchez, Long-distance radiative corrections to the di-pion tau lepton decay, Phys. Rev. D 74, 071301 (2006).

[66] A. Flores-Tlalpa, F. Flores-Báez, G. L. Castro, and G. T. Sánchez, Model-dependent radiative corrections to tau- $\longrightarrow$ pi- pi0 nu revisited, Nucl. Phys. B, Proc. Suppl. 169, 250 (2007).

[67] M. Davier, A. Hoecker, G. López Castro, B. Malaescu, X. H. Mo, G. T. Sánchez, P. Wang, C. Z. Yuan, and Z. Zhang, The discrepancy between tau and e+e- spectral functions revisited and the consequences for the muon magnetic anomaly, Eur. Phys. J. C 66, 127 (2010).

[68] M. Davier, A. Hoecker, B. Malaescu, and Z. Zhang, Reevaluation of the hadronic contributions to the muon g-2 and to alpha(MZ), Eur. Phys. J. C 71, 1515 (2011); Erratum, Eur. Phys. J. C 72, 1874 (2012).

[69] M. Davier, A. Höcker, B. Malaescu, C.-Z. Yuan, and Z. Zhang, Update of the ALEPH non-strange spectral functions from hadronic $\tau$ decays, Eur. Phys. J. C 74, 2803 (2014). 
[70] J. A. Miranda and P. Roig, Effective-field theory analysis of the $\tau^{-} \rightarrow \pi^{-} \pi^{0} \nu_{\tau}$ decays, J. High Energy Phys. 11 (2018) 038.

[71] V. Cirigliano, A. Falkowski, M. González-Alonso, and A. Rodríguez-Sánchez, Hadronic $\tau$ Decays as New Physics Probes in the LHC Era, Phys. Rev. Lett. 122, 221801 (2019).

[72] S. Gonzàlez-Solís, A. Miranda, J. Rendón, and P. Roig, Exclusive hadronic tau decays as probes of non-SM interactions, Phys. Lett. B 804, 135371 (2020).

[73] A. Crivellin, M. Hoferichter, C. Andrea Manzari, and M. Montull, Hadronic Vacuum Polarization: $(g-2)_{\mu}$ Versus Global Electroweak Fits, Phys. Rev. Lett. 125, 091801 (2020).

[74] A. Keshavarzi, W. J. Marciano, M. Passera, and A. Sirlin, Muon $g-2$ and $\Delta \alpha$ connection, Phys. Rev. D 102, 033002 (2020).

[75] E. de Rafael, On constraints between $\Delta \alpha_{\text {had }}\left(M_{Z}^{2}\right)$ and $\left(g_{\mu}-2\right)_{\mathrm{HVP}}$, Phys. Rev. D 102, 056025 (2020).

[76] B. Malaescu and M. Schott, Impact of correlations between $a_{\mu}$ and $\alpha_{\mathrm{QED}}$ on the EW fit, arXiv:2008.08107.

[77] F. Jegerlehner and R. Szafron, $\rho^{0}-\gamma$ mixing in the neutral channel pion form factor $F_{\pi}^{e}$ and its role in comparing $e^{+} e^{-}$with $\tau$ spectral functions, Eur. Phys. J. C 71, 1632 (2011).

[78] M. Bruno, T. Izubuchi, C. Lehner, and A. Meyer, On isospin breaking in $\tau$ decays for $(g-2)_{\mu}$ from lattice QCD, Proc. Sci., LATTICE2018 (2018) 135 [arXiv: 1811.00508].

[79] F. Jegerlehner, Muon g-2 theory: The hadronic part, EPJ Web Conf. 166, 00022 (2018).

[80] P. D. Ruiz-Femenía, A. Pich, and J. Portolés, Odd intrinsic parity processes within the resonance effective theory of QCD, J. High Energy Phys. 07 (2003) 003 [arXiv:hep-ph/ 0306157].

[81] V. Cirigliano, G. Ecker, M. Eidemuller, A. Pich, and J. Portolés, The $<\mathrm{VAP}>$ Green function in the resonance region, Phys. Lett. B 596, 96 (2004).

[82] V. Cirigliano, G. Ecker, M. Eidemuller, R. Kaiser, A. Pich, and J. Portolés, The $<\mathrm{SPP}>$ Green function and SU(3) breaking in K(13) decays, J. High Energy Phys. 04 (2005) 006.

[83] V. Cirigliano, G. Ecker, M. Eidemuller, Roland Kaiser, A. Pich, and J. Portolés, Towards a consistent estimate of the chiral low-energy constants, Nucl. Phys. B753, 139 (2006).

[84] Z.-H. Guo, Study of tau- $\rightarrow$ V P- nu(tau) in the framework of resonance chiral theory, Phys. Rev. D 78, 033004 (2008).

[85] D. G. Dumm, P. Roig, A. Pich, and J. Portolés, Hadron structure in tau $\longrightarrow$ KK pi nu (tau) decays, Phys. Rev. D 81, 034031 (2010).

[86] D. G. Dumm, P. Roig, A. Pich, and J. Portolés, tau $\longrightarrow$ pi pi pi nu(tau) decays and the a(1)(1260) off-shell width revisited, Phys. Lett. B 685, 158 (2010).

[87] Z.-H. Guo and P. Roig, One meson radiative tau decays, Phys. Rev. D 82, 113016 (2010).

[88] K. Kampf and J. Novotný, Resonance saturation in the odd-intrinsic parity sector of low-energy QCD, Phys. Rev. D 84, 014036 (2011).
[89] D. G. Dumm and P. Roig, Resonance Chiral Lagrangian analysis of $\tau^{-} \rightarrow \eta^{(\prime)} \pi^{-} \pi^{0} \nu_{\tau}$ decays, Phys. Rev. D 86, 076009 (2012).

[90] Y.-H. Chen, Z.-H. Guo, and H.-Q. Zheng, Study of $\eta-\eta^{\prime}$ mixing from radiative decay processes, Phys. Rev. D 85, 054018 (2012).

[91] P. Colangelo, J. J. Sanz-Cillero, and F. Zuo, Holography, chiral Lagrangian and form factor relations, J. High Energy Phys. 11 (2012) 012.

[92] L. Y. Dai, J. Portolés, and O. Shekhovtsova, Three pseudoscalar meson production in $e^{+} e^{-}$annihilation, Phys. Rev. D 88, 056001 (2013).

[93] A. Guevara, G. L. Castro, and P. Roig, Weak radiative pion vertex in $\tau^{-} \rightarrow \pi^{-} \nu_{\tau} \ell^{+} \ell^{-}$decays, Phys. Rev. D 88, 033007 (2013).

[94] P. Roig and J. J. S. Cillero, Consistent high-energy constraints in the anomalous QCD sector, Phys. Lett. B 733, 158 (2014).

[95] Y.-H. Chen, Z.-H. Guo, and H.-Q. Zheng, Radiative transition processes of light vector resonances in a chiral framework, Phys. Rev. D 90, 034013 (2014).

[96] P. Roig, A. Guevara, and G. L. Castro, $V V^{\prime} P$ form factors in resonance chiral theory and the $\pi-\eta-\eta^{\prime}$ light-by-light contribution to the muon $g-2$, Phys. Rev. D 89, 073016 (2014).

[97] Y.-H. Chen, D.-L. Yao, and H.-Q. Zheng, A study of $\rho-\omega$ mixing in resonance chiral theory, Commun. Theor. Phys. 69, 50 (2018).

[98] A. Guevara, P. Roig, and J. J. Sanz-Cillero, Pseudoscalar pole light-by-light contributions to the muon $(g-2)$ in resonance chiral theory, J. High Energy Phys. 06 (2018) 160.

[99] L.-Y. Dai, J. Fuentes-Martín, and J. Portolés, Scalarinvolved three-point Green functions and their phenomenology, Phys. Rev. D 99, 114015 (2019).

[100] T. Kadavý, K. Kampf, and J. Novotný, OPE of Green functions of chiral currents, arXiv:2006.13006.

[101] V. Mateu and J. Portolés, Form-factors in radiative pion decay, Eur. Phys. J. C 52, 325 (2007).

[102] B. Moussallam, A sum rule approach to the violation of Dashen's theorem, Nucl. Phys. B504, 381 (1997).

[103] S. Peris, M. Perrottet, and E. de Rafael, Matching long and short distances in large N(c) QCD, J. High Energy Phys. 05 (1998) 011.

[104] M. Knecht, S. Peris, M. Perrottet, and E. de Rafael, Decay of Pseudoscalars into Lepton Pairs and Large N(c) QCD, Phys. Rev. Lett. 83, 5230 (1999).

[105] S. Peris, B. Phily, and E. de Rafael, Tests of Large N(c) QCD from Hadronic tau Decay, Phys. Rev. Lett. 86, 14 (2001).

[106] M. Knecht and A. Nyffeler, Resonance estimates of $\mathrm{O}(\mathrm{p} * * 6)$ low-energy constants and QCD short distance constraints, Eur. Phys. J. C 21, 659 (2001).

[107] J. Bijnens, E. Gámiz, E. Lipartia, and J. Prades, QCD short distance constraints and hadronic approximations, J. High Energy Phys. 04 (2003) 055.

[108] B. Ananthanarayan and B. Moussallam, Four-point correlator constraints on electromagnetic chiral parameters and resonance effective Lagrangians, J. High Energy Phys. 06 (2004) 047. 
[109] A. Guevara, G. López-Castro, and P. Roig, $\tau^{-} \rightarrow \eta^{(\prime)} \pi^{-} \nu_{\tau} \gamma$ decays as backgrounds in the search for second class currents, Phys. Rev. D 95, 054015 (2017).

[110] J. Bijnens, G. Ecker, and J. Gasser, Radiative semileptonic kaon decays, Nucl. Phys. B396, 81 (1993).

[111] F. E. Low, Bremsstrahlung of Very Low-Energy Quanta in Elementary Particle Collisions, Phys. Rev. 110, 974 (1958).

[112] G. Hooft, A planar diagram theory for strong interactions, Nucl. Phys. B72, 461 (1974).

[113] G. Hooft, A two-dimensional model for Mesons, Nucl. Phys. B75, 461 (1974).

[114] E. Witten, Baryons in the 1/n expansion, Nucl. Phys. B160, 57 (1979).

[115] D. G. Dumm, A. Pich, and J. Portolés, The hadronic offshell width of meson resonances, Phys. Rev. D 62, 054014 (2000).

[116] I. M. Nugent, T. Przedzinski, P. Roig, O. Shekhovtsova, and Z. Was, Resonance chiral Lagrangian currents and experimental data for $\tau^{-} \rightarrow \pi^{-} \pi^{-} \pi^{+} \nu_{\tau}$, Phys. Rev. D 88, 093012 (2013).

[117] I. Rosell, J. J. Sanz-Cillero, and A. Pich, Quantum loops in the resonance chiral theory: The vector form-factor, J. High Energy Phys. 08 (2004) 042.

[118] I. Rosell, P. Ruiz-Femenía, and J. Portolés, One-loop renormalization of resonance chiral theory: Scalar and pseudoscalar resonances, J. High Energy Phys. 12 (2005) 020 .

[119] I. Rosell, J. J. Sanz-Cillero, and A. Pich, Towards a determination of the chiral couplings at NLO in $1 / \mathrm{N}(\mathrm{C})$ : L**r(8)(mu), J. High Energy Phys. 01 (2007) 039.

[120] J. Portolés, I. Rosell, and P. Ruiz-Femenía, Vanishing chiral couplings in the large- $\mathrm{N}(C)$ resonance theory, Phys. Rev. D 75, 114011 (2007).

[121] A. Pich, I. Rosell, and J. J. Sanz-Cillero, Form-factors and current correlators: Chiral couplings $\mathrm{L}(10) \mathrm{mu}) * * \mathrm{r}(\mathrm{mu})$ and $\mathrm{C}(87)^{* *} \mathrm{r}(\mathrm{mu})$ at NLO in $1 / \mathrm{N}(\mathrm{C})$, J. High Energy Phys. 07 (2008) 014.

[122] J. J. Sanz-Cillero, Renormalization group equations in resonance chiral theory, Phys. Lett. B 681, 100 (2009).

[123] A. Pich, I. Rosell, and J. J. Sanz-Cillero, The vector form factor at the next-to-leading order in $1 / \mathrm{N}(\mathrm{C})$ : Chiral couplings L9(mu) and C88(mu) - C90(mu), J. High Energy Phys. 02 (2011) 109.

[124] D G. Dumm and P. Roig, Dispersive representation of the pion vector form factor in $\tau \rightarrow \pi \pi \nu_{\tau}$ decays, Eur. Phys. J. C 73, 2528 (2013).

[125] S. Gonzàlez-Solís and P. Roig, A dispersive analysis of the pion vector form factor and $\tau^{-} \rightarrow K^{-} K_{S} \nu_{\tau}$ decay, Eur. Phys. J. C 79, 436 (2019).

[126] S. Weinberg, Phenomenological Lagrangians, Physica (Amsterdam) 96A, 327 (1979).

[127] J. Gasser and H. Leutwyler, Chiral perturbation theory to one loop, Ann. Phys. (N.Y.) 158, 142 (1984).

[128] J. Gasser and H. Leutwyler, Chiral perturbation theory: Expansions in the mass of the strange quark, Nucl. Phys. B250, 465 (1985).

[129] J. Bijnens, G. Colangelo, and G. Ecker, The mesonic chiral Lagrangian of order $\mathrm{p}^{* * 6}$, J. High Energy Phys. 02 (1999) 020 .
[130] J. Bijnens, L. Girlanda, and P. Talavera, The anomalous chiral Lagrangian of order p**6, Eur. Phys. J. C 23, 539 (2002).

[131] J. Wess and B. Zumino, Consequences of anomalous ward identities, Phys. Lett. 37B, 95 (1971).

[132] E. Witten, Global aspects of current algebra, Nucl. Phys. B223, 422 (1983).

[133] A. Pich, Colorless mesons in a polychromatic world, in Phenomenology of Large N(c) QCD. Proceedings, Tempe, USA (World Scientific, Singapore, 2002), pp. 239-258, https://doi.org/10.1142/9789812776914_0023.

[134] S. Weinberg, Precise Relations between the Spectra of Vector and Axial Vector Mesons, Phys. Rev. Lett. 18, 507 (1967).

[135] M.F. L. Golterman and S. Peris, The 7/11 rule: An estimate of m(rho)/f(pi), Phys. Rev. D 61, 034018 (2000).

[136] M. Jamin, J. A. Oller, and A. Pich, S wave K pi scattering in chiral perturbation theory with resonances, Nucl. Phys. B587, 331 (2000).

[137] M. Jamin, J. A. Oller, and A. Pich, Strangeness changing scalar form-factors, Nucl. Phys. B622, 279 (2002).

[138] S. J. Brodsky and G. R. Farrar, Scaling Laws at Large Transverse Momentum, Phys. Rev. Lett. 31, 1153 (1973).

[139] G. P. Lepage and S. J. Brodsky, Exclusive processes in perturbative quantum chromodynamics, Phys. Rev. D 22, 2157 (1980).

[140] Y.-H. Chen, Z.-H. Guo, and B.-S. Zou, Unified study of $J / \psi \rightarrow P V, P \gamma^{(*)}$ and light hadron radiative processes, Phys. Rev. D 91, 014010 (2015).

[141] T. H. Burnett and N. M. Kroll, Extension of the Low Soft Photon Theorem, Phys. Rev. Lett. 20, 86 (1968).

[142] A. Flores-Tlalpa, Modelo de dominancia de mesones para decaimientos semileptónicos de sabores pesados., Ph.D. thesis, CINVESTAV, IPN, 2008.

[143] F. Guerrero and A. Pich, Effective field theory description of the pion form-factor, Phys. Lett. B 412, 382 (1997).

[144] J. J. Sakurai, Theory of strong interactions, Ann. Phys. (N.Y.) 11, 1 (1960).

[145] M. Gourdin and E. De Rafael, Hadronic contributions to the muon g-factor, Nucl. Phys. B10, 667 (1969).

[146] A. Sirlin, Radiative corrections to $g(v) / g(m u)$ in simple extensions of the $\operatorname{su}(2) \times \mathrm{u}(1)$ gauge model, Nucl. Phys. A71, 29 (1974).

[147] A. Sirlin, Current algebra formulation of radiative corrections in gauge theories and the universality of the weak interactions, Rev. Mod. Phys. 50, 573 (1978); Erratum, Rev. Mod. Phys. 50, 905 (1978).

[148] A. Sirlin, Large $m(W), m(Z)$ behavior of the O(alpha) corrections to semileptonic processes Mediated by W, Nucl. Phys. B196, 83 (1982).

[149] W. J. Marciano and A. Sirlin, Radiative Corrections to beta Decay and the Possibility of a Fourth Generation, Phys. Rev. Lett. 56, 22 (1986).

[150] W. J. Marciano and A. Sirlin, Electroweak Radiative Corrections to tau Decay, Phys. Rev. Lett. 61, 1815 (1988).

[151] E. Braaten and C.-S. Li, Electroweak radiative corrections to the semihadronic decay rate of the tau lepton, Phys. Rev. D 42, 3888 (1990).

[152] W. J. Marciano and A. Sirlin, Radiative Corrections to pi (lepton 2) Decays, Phys. Rev. Lett. 71, 3629 (1993). 
[153] J. Erler, Electroweak radiative corrections to semileptonic tau decays, Rev. Mex. Fis. 50, 200 (2004), http://www .ejournal.unam.mx/revmexfis/no502/RMF50216.pdf.

[154] J. S. Schwinger, Particles, Sources, and Fields (AddisonWesley, Redwood City, 1989), Vol. 3, https://inspirehep .net/literature/281678.

[155] M. Drees and K.-I. Hikasa, Scalar top production in e+ eannihilation, Phys. Lett. B 252, 127 (1990).

[156] M. Tanabashi et al., Review of particle physics, Phys. Rev. D 98, 030001 (2018).

[157] P. A. Zyla et al., Review of particle physics, Prog. Theor. Exp. Phys. 2020, 083C01 (2020).

[158] M. Fujikawa et al., High-statistics study of the tau- $\rightarrow$ pi- pi0 nu(tau) decay, Phys. Rev. D 78, 072006 (2008).

[159] S. Schael et al., Branching ratios and spectral functions of tau decays: Final ALEPH measurements and physics implications, Phys. Rep. 421, 191 (2005).

[160] D. Babusci et al., Precision measurement of $\sigma\left(e^{+} e^{-} \rightarrow\right.$ $\left.\pi^{+} \pi^{-} \gamma\right) / \sigma\left(e^{+} e^{-} \rightarrow \mu^{+} \mu^{-} \gamma\right)$ and determination of the $\pi^{+} \pi^{-}$ contribution to the muon anomaly with the KLOE detector, Phys. Lett. B 720, 336 (2013).

[161] M. Ablikim et al., Erratum to "Measurement of the $e^{+} e^{-} \rightarrow \pi^{+} \pi^{-}$cross section between 600 and $900 \mathrm{MeV}$ using initial state radiation", arXiv:2009.05011.
[162] M. Benayoun, P. David, L. DelBuono, and F. Jegerlehner, Upgraded breaking of the HLS model: A full solution to the $\tau^{-} e^{+} e^{-}$and $\phi$ decay issues and its consequences on $\mathrm{g}-2$ VMD estimates, Eur. Phys. J. C 72, 1848 (2012).

[163] M. Benayoun, P. David, L. DelBuono, and F. Jegerlehner, An update of the HLS estimate of the muon g-2, Eur. Phys. J. C 73, 2453 (2013).

[164] M. Benayoun, P. David, L. DelBuono, and F. Jegerlehner, Muon $g-2$ estimates: Can one trust effective Lagrangians and global fits?, Eur. Phys. J. C 75, 613 (2015).

[165] M. Benayoun, L. Delbuono, and F. Jegerlehner, BHLS 2 , A new breaking of the HLS model and its phenomenology, Eur. Phys. J. C 80, 81 (2020); Erratum, Eur. Phys. J. C 80, 244 (2020).

[166] S. Anderson et al., Hadronic structure in the decay tau- $\longrightarrow$ pi- pi0 neutrino(tau), Phys. Rev. D 61, 112002 (2000).

[167] K. Ackerstaff et al., Measurement of the strong coupling constant alpha(s) and the vector and axial vector spectral functions in hadronic tau decays, Eur. Phys. J. C 7, 571 (1999).

[168] S.-Z. Jiang, Z.-L. Wei, Q.-S. Chen, and Q. Wang, Computation of the $O\left(p^{6}\right)$ order low-energy constants: An update, Phys. Rev. D 92, 025014 (2015). 